\title{
Synthesis and synthetic application of chloro- and
}

bromofuroxans

Ryosuke Matsubara, ${ }^{\text {a }}$ Akihiro Ando, ${ }^{a}$ Hayu Hasebe, ${ }^{a}$ Hojin Kim, ${ }^{a}$ Takao Tsuneda, ${ }^{\mathrm{b}}$ Masahiko Hayashi $^{\mathrm{a}}$

a Department of Chemistry, Graduate School of Science, Kobe University, Nada-ku, Kobe, Hyogo 6578501 (Japan)

${ }^{\mathrm{b}}$ Graduate School of Science, Technology and Innovation, Kobe University, Nada-ku, Kobe, Hyogo 6578501 (Japan).

\section{Table of Contents}

$\begin{array}{ll}\text { 1. DFT calculations } & \mathrm{S} 2\end{array}$

$\begin{array}{ll}\text { 2. Crystallographic details } & \text { S15 }\end{array}$

3. NMR spectra $\quad$ S33 


\section{DFT calculations}

(a)
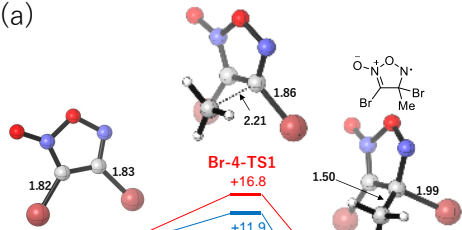

(1)

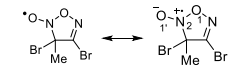

$\begin{array}{cc}{ }^{\mathrm{Br}} \mathrm{Me}_{\mathrm{Br}} & { }_{\mathrm{Br}}{ }_{\mathrm{Me}} \mathrm{Br} \\ \text { nitroxyl radical } & \mathrm{Br} \text {-3-INT }\end{array}$

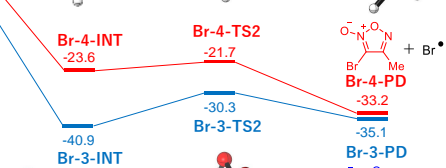

(b)
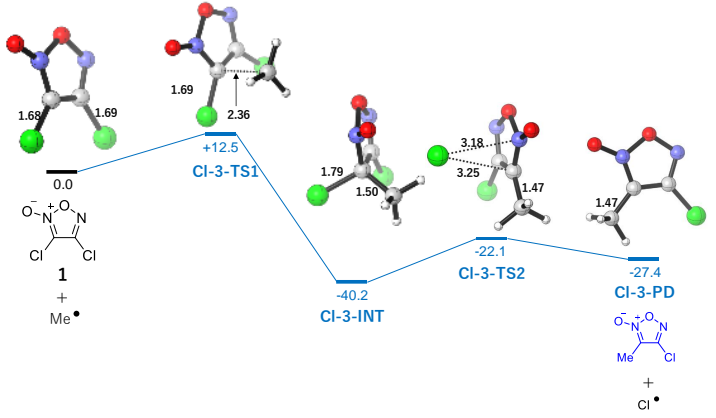

\section{Dibromofuroxan}

SCF Done: $\quad \mathrm{E}(\mathrm{RLC}-\mathrm{B}+\mathrm{HF}-\mathrm{LYP})=-5477.83015899$ a.u.

Sum of electronic and thermal Free Energies $=-5477.831616$ a.u.

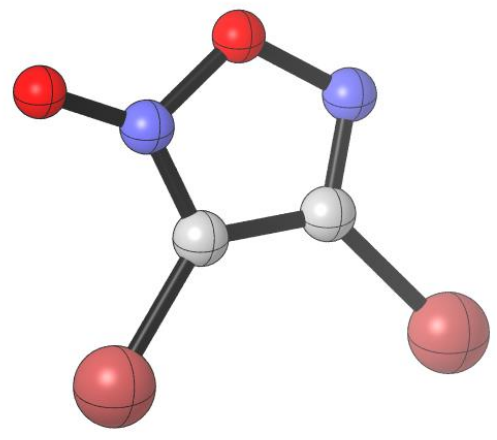

\begin{tabular}{ccrcrr} 
Center & Atomic & Atomic & \multicolumn{3}{c}{ Coordinates (Angstroms) } \\
Number & Number & Type & $\mathrm{X}$ & $\mathrm{Y}$ & $\mathrm{Z}$ \\
-1 & 6 & 0 & -0.669791 & 0.738914 & -0.000458 \\
2 & 6 & 0 & 0.651005 & 0.251934 & -0.000688 \\
3 & 8 & 0 & 0.606890 & 2.424764 & -0.000165 \\
4 & 7 & 0 & -0.687571 & 2.023975 & -0.001167 \\
5 & 7 & 0 & 1.424874 & 1.312307 & 0.000395 \\
6 & 8 & 0 & 2.625627 & 1.477756 & 0.001128 \\
7 & 35 & 0 & -2.188953 & -0.284946 & 0.000307 \\
8 & 35 & 0 & 1.305852 & -1.444174 & -0.000176
\end{tabular}




\section{Methyl radical}

SCF Done: $\quad \mathrm{E}(\mathrm{ULC}-\mathrm{B}+\mathrm{HF}-\mathrm{LYP})=-39.6928229469$ a.u.

Sum of electronic and thermal Free Energies $=-39.681325$ a.u.

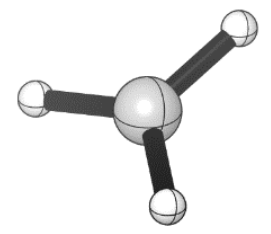

\begin{tabular}{cccccc} 
Center & $\begin{array}{c}\text { Atomic } \\
\text { Number }\end{array}$ & $\begin{array}{r}\text { Atomic } \\
\text { Number }\end{array}$ & \multicolumn{3}{c}{ Coordinates (Angstroms) } \\
-1 & 6 & 0 & 0.000000 & 0.000000 & 0.000014 \\
2 & 1 & 0 & 0.000000 & 1.083463 & -0.000027 \\
3 & 1 & 0 & 0.938306 & -0.541731 & -0.000027 \\
4 & 1 & 0 & -0.938306 & -0.541731 & -0.000027
\end{tabular}

\section{Br-4-TS1}

SCF Done: $\quad E(U L C-B+H F-L Y P)=-5517.51541981$ a.u.

Sum of electronic and thermal Free Energies $=-5517.486119$ a.u.

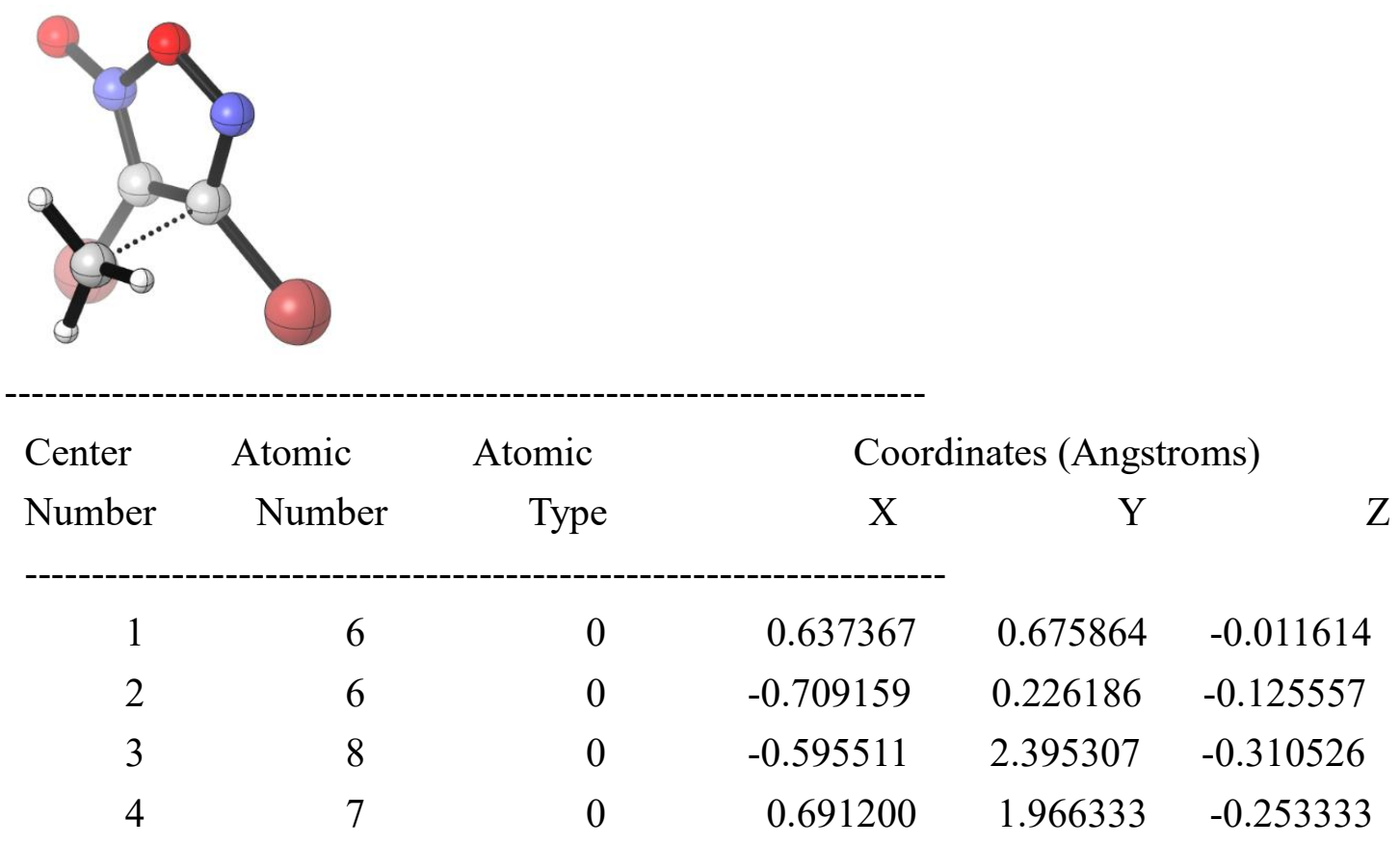




$\begin{array}{rrrrrr}5 & 7 & 0 & -1.449955 & 1.290762 & -0.266435 \\ 6 & 8 & 0 & -2.645675 & 1.490959 & -0.346922 \\ 7 & 6 & 0 & 0.746486 & 0.630190 & 2.199966 \\ 8 & 1 & 0 & 1.763320 & 0.976817 & 2.352955 \\ 9 & 1 & 0 & -0.048329 & 1.327197 & 2.450355 \\ 10 & 1 & 0 & 0.552667 & -0.421361 & 2.391299 \\ 11 & 35 & 0 & 2.111459 & -0.408725 & -0.333110 \\ 12 & 35 & 0 & -1.399317 & -1.447443 & 0.028155\end{array}$

\section{Br-4-INT}

SCF Done: $\quad$ E(ULC-B+HF-LYP $)=-5517.58468446$ a.u.

Sum of electronic and thermal Free Energies $=-5517.550626$ a.u.

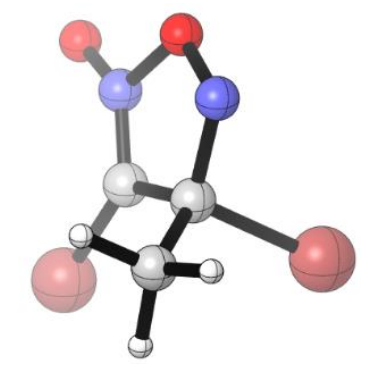

\begin{tabular}{rrrrrr} 
Center & Atomic & Atomic & \multicolumn{3}{c}{ Coordinates (Angstroms) } \\
Number & Number & Type & $\mathrm{X}$ & $\mathrm{Y}$ & $\mathrm{Z}$ \\
-1 & 6 & 0 & 0.723297 & 0.342148 & 0.706588 \\
2 & 6 & 0 & -0.645987 & 0.346433 & 0.168982 \\
3 & 8 & 0 & 0.190320 & 2.384157 & -0.016484 \\
4 & 7 & 0 & 1.117149 & 1.718229 & 0.656681 \\
5 & 7 & 0 & -0.950368 & 1.512173 & -0.273963 \\
6 & 8 & 0 & -1.897409 & 2.019898 & -0.825822 \\
7 & 6 & 0 & 0.932245 & -0.269729 & 2.063268 \\
8 & 1 & 0 & 1.976707 & -0.168990 & 2.359804 \\
9 & 1 & 0 & 0.302953 & 0.247343 & 2.791508 \\
10 & 1 & 0 & 0.669949 & -1.328278 & 2.048751 \\
11 & 35 & 0 & -1.773280 & -1.072519 & 0.010962 \\
12 & 35 & 0 & 1.872774 & -0.616294 & -0.604496
\end{tabular}




\section{Br-4-TS2}

SCF Done: $\quad E(U L C-B+H F-L Y P)=-5517.58099450$ a.u.

Sum of electronic and thermal Free Energies $=-5517.547581$ a.u.

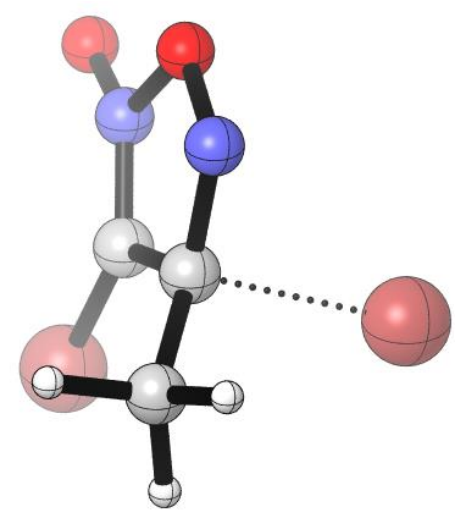

\begin{tabular}{rrrrrr} 
Center & Atomic & Atomic & \multicolumn{3}{c}{ Coordinates (Angstroms) } \\
Number & Number & Type & $\mathrm{X}$ & $\mathrm{Y}$ & $\mathrm{Z}$ \\
\hline 1 & 6 & 0 & 0.591659 & 0.297293 & 0.939485 \\
2 & 6 & 0 & -0.673448 & 0.362162 & 0.256847 \\
3 & 8 & 0 & 0.341391 & 2.303830 & 0.141044 \\
4 & 7 & 0 & 1.104667 & 1.560754 & 0.933292 \\
5 & 7 & 0 & -0.807155 & 1.548141 & -0.254219 \\
6 & 8 & 0 & -1.622908 & 2.103310 & -0.953198 \\
7 & 6 & 0 & 0.857348 & -0.578211 & 2.113893 \\
8 & 1 & 0 & 1.905762 & -0.512131 & 2.403138 \\
9 & 1 & 0 & 0.237079 & -0.240538 & 2.948387 \\
10 & 1 & 0 & 0.613003 & -1.615314 & 1.886984 \\
11 & 35 & 0 & -1.872898 & -0.967419 & -0.053232 \\
12 & 35 & 0 & 1.894623 & -0.607977 & -0.671229
\end{tabular}




\section{Br-4-PD}

SCF Done: $\quad E($ RLC-B+HF-LYP $)=-2946.33316377$ a.u.

Sum of electronic and thermal Free Energies $=-2946.294708$ a.u.

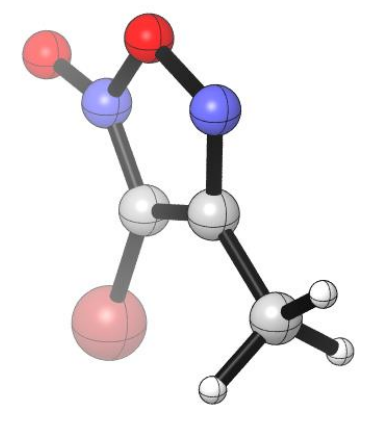

\begin{tabular}{rrrrrr} 
Center & Atomic & Atomic & \multicolumn{3}{c}{ Coordinates (Angstroms) } \\
Number & Number & Type & $\mathrm{X}$ & $\mathrm{Y}$ & $\mathrm{Z}$ \\
---------1 & & \\
1 & 6 & 0 & -0.158901 & -0.034236 & -0.000145 \\
2 & 6 & 0 & -1.149874 & 0.973405 & 0.000255 \\
3 & 8 & 0 & -2.129586 & -0.927479 & 0.000227 \\
4 & 7 & 0 & -0.780671 & -1.187069 & -0.000054 \\
5 & 7 & 0 & -2.317019 & 0.421206 & -0.000358 \\
6 & 6 & 0 & -0.937226 & 2.433736 & 0.000087 \\
7 & 1 & 0 & -0.368677 & 2.736181 & 0.882707 \\
8 & 1 & 0 & -0.375636 & 2.736306 & -0.886936 \\
9 & 1 & 0 & -1.897281 & 2.949761 & 0.003985 \\
10 & 8 & 0 & -0.410959 & -2.348076 & 0.000030 \\
11 & 35 & 0 & 1.660737 & 0.083023 & -0.000003
\end{tabular}

\section{Br radical}

SCF Done: $\quad E(U L C-B+H F-L Y P)=-2571.25435811$ a.u.

Sum of electronic and thermal Free Energies $=-2571.271188$ a.u.

\begin{tabular}{cccccr} 
Center & Atomic & Atomic & \multicolumn{3}{c}{ Coordinates (Angstroms) } \\
Number & Number & Type & X & Y & Z \\
-1 & 35 & 0 & 0.000000 & 0.000000 & 0.000000
\end{tabular}




\section{Br-3-TS1}

SCF Done: $\quad E(U L C-B+H F-L Y P)=-5517.52173812$ a.u.

Sum of electronic and thermal Free Energies $=-5517.494031$ a.u.

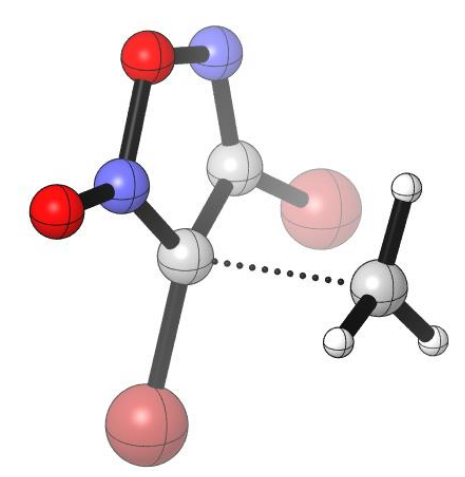

\begin{tabular}{rrrrrr} 
Center & Atomic & Atomic & \multicolumn{3}{c}{ Coordinates (Angstroms) } \\
Number & Number & Type & $\mathrm{X}$ & $\mathrm{Y}$ & $\mathrm{Z}$ \\
-1 & 6 & 0 & 0.703301 & 0.735259 & -0.180185 \\
2 & 6 & 0 & -0.614110 & 0.227617 & -0.024723 \\
3 & 8 & 0 & -0.599877 & 2.399049 & -0.369323 \\
4 & 7 & 0 & 0.700844 & 2.004103 & -0.351846 \\
5 & 7 & 0 & -1.408512 & 1.267880 & -0.263230 \\
6 & 8 & 0 & -2.616210 & 1.408423 & -0.298870 \\
7 & 6 & 0 & -0.615786 & 0.262388 & 2.321682 \\
8 & 1 & 0 & -1.676624 & 0.068123 & 2.443217 \\
9 & 1 & 0 & 0.072507 & -0.547777 & 2.539662 \\
10 & 1 & 0 & -0.269191 & 1.276331 & 2.498978 \\
11 & 35 & 0 & -1.212982 & -1.494268 & -0.235909 \\
12 & 35 & 0 & 2.233418 & -0.263215 & -0.064988
\end{tabular}




\section{Br-3-INT}

SCF Done: $\quad E(U L C-B+H F-L Y P)=-5517.61193803$ a.u.

Sum of electronic and thermal Free Energies $=-5517.578168$ a.u.

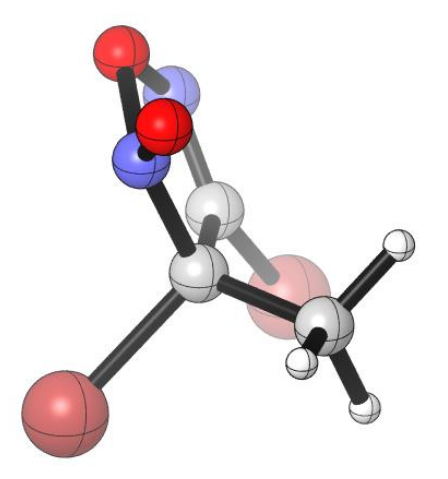

\begin{tabular}{rrrrrr} 
Center & Atomic & Atomic & \multicolumn{3}{c}{ Coordinates (Angstroms) } \\
Number & Number & Type & $\mathrm{X}$ & $\mathrm{Y}$ & $\mathrm{Z}$ \\
-1 & 6 & 0 & 0.585051 & 0.719013 & -0.193901 \\
2 & 6 & 0 & -0.650079 & 0.179991 & 0.431671 \\
3 & 8 & 0 & -0.923114 & 2.036486 & -0.964271 \\
4 & 7 & 0 & 0.397687 & 1.713284 & -0.946286 \\
5 & 7 & 0 & -1.603010 & 1.146254 & -0.076734 \\
6 & 8 & 0 & -2.565482 & 1.602548 & 0.531063 \\
7 & 6 & 0 & -0.669089 & 0.088565 & 1.930780 \\
8 & 1 & 0 & -1.650009 & -0.241898 & 2.272139 \\
9 & 1 & 0 & 0.082154 & -0.624277 & 2.271388 \\
10 & 1 & 0 & -0.454069 & 1.070433 & 2.359056 \\
11 & 35 & 0 & -1.021181 & -1.563636 & -0.360019 \\
12 & 35 & 0 & 2.243257 & -0.015184 & 0.094674
\end{tabular}




\section{Br-3-TS2}

SCF Done: $\quad E(U L C-B+H F-L Y P)=-5517.59397054$ a.u.

Sum of electronic and thermal Free Energies $=-5517.561359$ a.u.

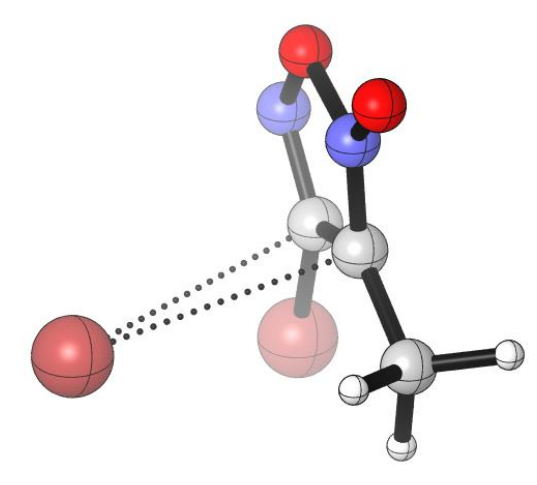

\begin{tabular}{rrrrrr} 
Center & Atomic & Atomic & \multicolumn{3}{c}{ Coordinates (Angstroms) } \\
Number & Number & Type & $\mathrm{X}$ & $\mathrm{Y}$ & $\mathrm{Z}$ \\
-1 & 6 & 0 & -0.007068 & 1.024980 & -0.260492 \\
2 & 6 & 0 & -1.005299 & 0.626165 & 0.653804 \\
3 & 8 & 0 & -1.819900 & 1.064159 & -1.335339 \\
4 & 7 & 0 & -0.488953 & 1.279141 & -1.427008 \\
5 & 7 & 0 & -2.116842 & 0.661740 & -0.045387 \\
6 & 8 & 0 & -3.278771 & 0.415653 & 0.223161 \\
7 & 6 & 0 & -0.981845 & 0.247215 & 2.077869 \\
8 & 1 & 0 & -1.414861 & -0.745067 & 2.224273 \\
9 & 1 & 0 & 0.044653 & 0.241834 & 2.444702 \\
10 & 1 & 0 & -1.557423 & 0.960989 & 2.672672 \\
11 & 35 & 0 & 0.319427 & -2.193531 & -0.185085 \\
12 & 35 & 0 & 1.792654 & 1.128600 & 0.100384
\end{tabular}




\section{Br-3-PD}

SCF Done: $\quad E(R L C-B+H F-L Y P)=-2946.33434055$ a.u.

Sum of electronic and thermal Free Energies $=-2946.297802$ a.u.

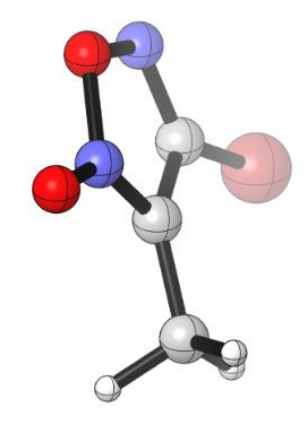

\begin{tabular}{rrrrrr} 
Center & Atomic & Atomic & \multicolumn{3}{c}{ Coordinates (Angstroms) } \\
Number & Number & Type & $\mathrm{X}$ & $\mathrm{Y}$ & $\mathrm{Z}$ \\
-1 & 6 & 0 & 0.138969 & -0.349597 & -0.000016 \\
2 & 6 & 0 & -0.904324 & 0.598189 & -0.000056 \\
3 & 8 & 0 & -1.651724 & -1.462754 & -0.000040 \\
4 & 7 & 0 & -0.303807 & -1.558500 & -0.000002 \\
5 & 7 & 0 & -1.998710 & -0.123039 & 0.000025 \\
6 & 8 & 0 & -3.186172 & 0.159701 & 0.000052 \\
7 & 6 & 0 & -0.936071 & 2.070723 & -0.000006 \\
8 & 1 & 0 & -1.453181 & 2.443321 & 0.887756 \\
9 & 1 & 0 & 0.079472 & 2.466464 & -0.004429 \\
10 & 1 & 0 & -1.460603 & 2.443003 & -0.883521 \\
11 & 35 & 0 & 1.938961 & 0.026472 & 0.000011
\end{tabular}

\section{Dichlorofuroxan}

SCF Done: $\quad E(R L C-B+H F-L Y P)=-1255.07601305$ a.u.

Sum of electronic and thermal Free Energies $=-1255.074308$ a.u.

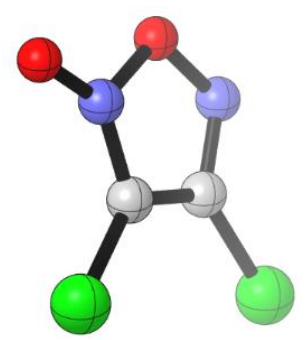




\begin{tabular}{crrrrr} 
Center & Atomic & Atomic & \multicolumn{3}{c}{ Coordinates (Angstroms) } \\
Number & Number & Type & $\mathrm{X}$ & $\mathrm{Y}$ & $\mathrm{Z}$ \\
--------1 & & \\
1 & 6 & 0 & -0.800282 & -0.489111 & -0.000271 \\
2 & 6 & 0 & 0.363212 & 0.307685 & -0.000030 \\
3 & 8 & 0 & 0.850686 & -1.809815 & 0.000052 \\
4 & 7 & 0 & -0.501575 & -1.739475 & 0.000386 \\
5 & 7 & 0 & 1.373430 & -0.530950 & -0.000214 \\
6 & 8 & 0 & 2.578619 & -0.397163 & -0.000084 \\
7 & 17 & 0 & -2.392454 & 0.067251 & -0.000061 \\
8 & 17 & 0 & 0.573924 & 1.970241 & 0.000111
\end{tabular}

\section{Cl-3-TS1}

SCF Done: $\quad E(U L C-B+H F-L Y P)=-1294.76654043$ a.u.

Sum of electronic and thermal Free Energies $=-1294.735633$ a.u.

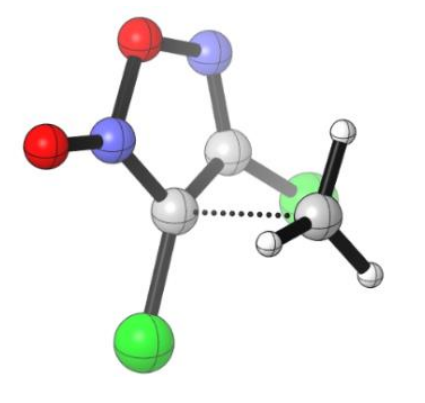

\begin{tabular}{cccccc}
$\begin{array}{c}\text { Center } \\
\text { Number }\end{array}$ & $\begin{array}{r}\text { Atomic } \\
\text { Number }\end{array}$ & $\begin{array}{r}\text { Atomic } \\
\text { Type }\end{array}$ & \multicolumn{3}{c}{$\begin{array}{c}\text { Coordinates (Angstroms) } \\
\text { X }\end{array}$} \\
-1 & 6 & 0 & -0.852607 & -0.538373 & -0.156395 \\
2 & 6 & 0 & 0.326557 & 0.257876 & -0.123903 \\
3 & 8 & 0 & 0.779810 & -1.892758 & -0.168406 \\
4 & 7 & 0 & -0.574522 & -1.788596 & -0.151025 \\
5 & 7 & 0 & 1.324283 & -0.614423 & -0.256994 \\
6 & 8 & 0 & 2.533029 & -0.496937 & -0.325435 \\
7 & 6 & 0 & 0.415322 & 0.545096 & 2.214602 \\
8 & 1 & 0 & 1.436244 & 0.913368 & 2.224089
\end{tabular}




$\begin{array}{rrrrrr}9 & 1 & 0 & -0.385028 & 1.263011 & 2.361486 \\ 10 & 1 & 0 & 0.244288 & -0.475547 & 2.543343 \\ 11 & 17 & 0 & 0.529974 & 1.875956 & -0.567529 \\ 12 & 17 & 0 & -2.434808 & 0.044648 & -0.134111\end{array}$

\section{Cl-3-INT}

SCF Done: $\quad E(U L C-B+H F-L Y P)=-1294.85723195$ a.u.

Sum of electronic and thermal Free Energies $=-1294.819828$ a.u.

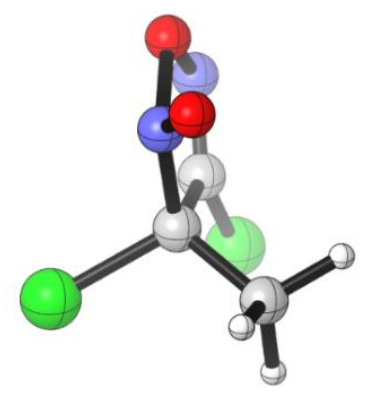

\begin{tabular}{rrrrrr} 
Center & Atomic & Atomic & \multicolumn{3}{c}{ Coordinates (Angstroms) } \\
Number & Number & Type & $\mathrm{X}$ & $\mathrm{Y}$ & $\mathrm{Z}$ \\
-1 & 6 & 0 & -0.771374 & -0.581118 & -0.050965 \\
2 & 6 & 0 & 0.329205 & 0.381860 & 0.242705 \\
3 & 8 & 0 & 0.965292 & -1.700952 & -0.628349 \\
4 & 7 & 0 & -0.388932 & -1.683293 & -0.528913 \\
5 & 7 & 0 & 1.461012 & -0.469492 & -0.096968 \\
6 & 8 & 0 & 2.522594 & -0.526124 & 0.514846 \\
7 & 6 & 0 & 0.409194 & 0.892891 & 1.653152 \\
8 & 1 & 0 & 1.315332 & 1.485632 & 1.779488 \\
9 & 1 & 0 & -0.457831 & 1.517572 & 1.869414 \\
10 & 1 & 0 & 0.428917 & 0.049804 & 2.346935 \\
11 & 17 & 0 & 0.255707 & 1.742219 & -0.919307 \\
12 & 17 & 0 & -2.402543 & -0.232143 & 0.226601
\end{tabular}




\section{Cl-3-TS2}

SCF Done: $\quad$ E $($ ULC-B+HF-LYP $)=-1294.82454566$ a.u.

Sum of electronic and thermal Free Energies $=-1294.791024$ a.u.

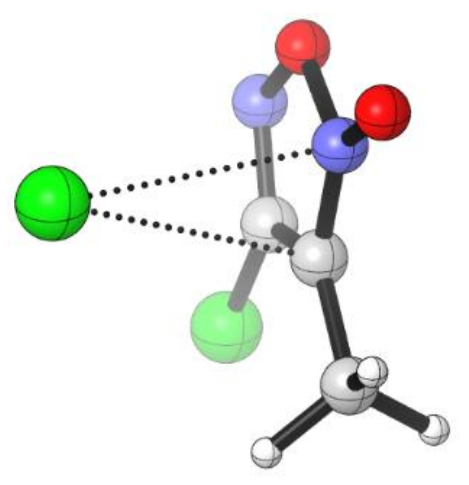

\begin{tabular}{rrrrrr} 
Center & Atomic & Atomic & \multicolumn{3}{c}{ Coordinates (Angstroms) } \\
Number & Number & Type & $\mathrm{X}$ & $\mathrm{Y}$ & $\mathrm{Z}$ \\
\hline 1 & 6 & 0 & -1.144213 & -0.177671 & -0.260800 \\
2 & 6 & 0 & -0.150708 & -0.402613 & 0.717121 \\
3 & 8 & 0 & 0.311036 & -1.383709 & -1.189554 \\
4 & 7 & 0 & -0.868948 & -0.753249 & -1.378014 \\
5 & 7 & 0 & 0.732113 & -1.162451 & 0.110541 \\
6 & 8 & 0 & 1.791324 & -1.660884 & 0.449770 \\
7 & 6 & 0 & -0.024711 & 0.016520 & 2.124421 \\
8 & 1 & 0 & 0.975881 & -0.225039 & 2.484558 \\
9 & 1 & 0 & -0.189064 & 1.091254 & 2.224089 \\
10 & 1 & 0 & -0.756922 & -0.502468 & 2.748215 \\
11 & 17 & 0 & 2.085148 & 1.664256 & -0.431140 \\
12 & 17 & 0 & -2.554156 & 0.734890 & -0.048315
\end{tabular}




\section{Cl-3-PD}

SCF Done: $\quad E($ RLC-B + HF-LYP $)=-834.958417419$ a.u.

Sum of electronic and thermal Free Energies $=-834.919382$ a.u.

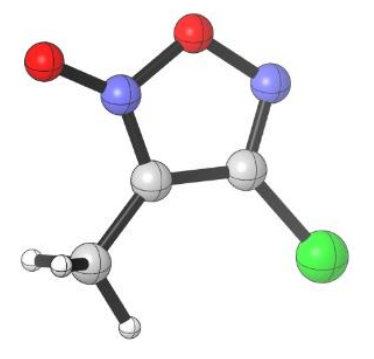

\begin{tabular}{|c|c|c|c|c|c|}
\hline \multirow{2}{*}{$\begin{array}{l}\text { Center } \\
\text { Number }\end{array}$} & \multirow{2}{*}{$\begin{array}{l}\text { Atomic } \\
\text { Number }\end{array}$} & \multirow{2}{*}{$\begin{array}{l}\text { Atomic } \\
\text { Type }\end{array}$} & \multicolumn{3}{|c|}{ Coordinates (Angstroms) } \\
\hline & & & $X$ & Y & Z \\
\hline 1 & 6 & 0 & 0.696526 & -0.314345 & -0.000030 \\
\hline 2 & 6 & 0 & -0.372863 & 0.605510 & -0.000069 \\
\hline 3 & 8 & 0 & -1.060215 & -1.477069 & 0.000042 \\
\hline 4 & 7 & 0 & 0.289884 & -1.535909 & -0.000017 \\
\hline 5 & 7 & 0 & -1.445751 & -0.147553 & 0.000040 \\
\hline 6 & 8 & 0 & -2.640975 & 0.100068 & 0.000104 \\
\hline 7 & 6 & 0 & -0.449700 & 2.076497 & -0.000022 \\
\hline 8 & 1 & 0 & -0.979450 & 2.431568 & 0.887544 \\
\hline 9 & 1 & 0 & 0.552464 & 2.504878 & -0.004294 \\
\hline 10 & 1 & 0 & -0.986705 & 2.431075 & -0.883391 \\
\hline 11 & 17 & 0 & 2.345324 & 0.072162 & -0.000027 \\
\hline
\end{tabular}

\section{Cl radical}

SCF Done: $\quad E(U L C-B+H F-L Y P)=-459.864286057$ a.u.

Sum of electronic and thermal Free Energies $=-459.879963$ a.u.

\begin{tabular}{cccccr} 
Center & Atomic & Atomic & \multicolumn{3}{c}{ Coordinates (Angstroms) } \\
Number & Number & Type & $X$ & $Y$ & Z \\
------------------------------------------------------------ & & \\
1 & 17 & 0 & 0.000000 & 0.000000 & 0.000000
\end{tabular}




\section{Crystallographic details}

Single crystals of 2 were obtained by recrystallization from hexane/1,2-dichloroethane by vapor diffusion. Single crystal X-ray diffraction analyses were conducted using Bruker Apex-II diffractometer equipped with a CCD detector using monochromatic Mo- $K \alpha$ radiation $(0.71069 \AA)$. All non-hydrogen atoms were refined with anisotropic atomic displacement parameters. Hydrogen atoms attached to carbon atoms were treated as riding atoms, using isotropic displacement parameters.

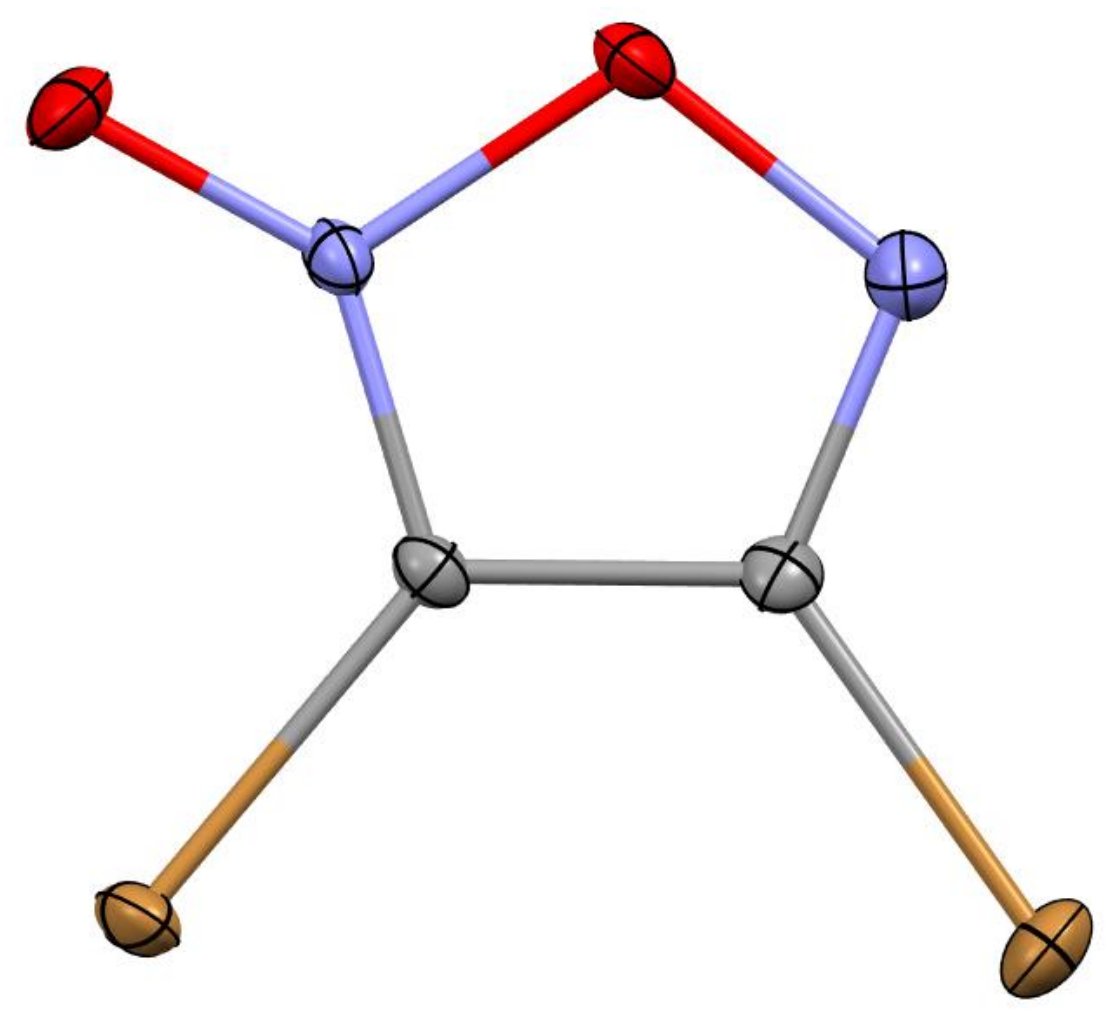

Asymmetric unit in the crystal structure of 2, thermal ellipsoids are drawn at 50\% probability level. Hydrogen atoms are drawn as fixed-size spheres of radius $0.20 \AA$.

Table S1. Crystal data and structure refinement details.

\begin{tabular}{|l|l|}
\hline Empirical formula & $\mathrm{C}_{2} \mathrm{Br}_{2} \mathrm{~N}_{2} \mathrm{O}_{2}$ \\
\hline Formula weight & 243.84 \\
\hline Temperature/K & 90 \\
\hline Crystal system & orthorhombic \\
\hline Space group & $P 2_{1} 2_{1} 2_{1}$ \\
\hline$a / \AA$ & $5.9208(10)$ \\
\hline$b / \AA$ & $7.7879(13)$ \\
\hline$c / \AA$ & $12.680(2)$ \\
\hline
\end{tabular}




\begin{tabular}{|l|l|}
\hline$\alpha{ }^{\circ}$ & 90 \\
\hline$\beta /^{\circ}$ & 90 \\
\hline$\gamma{ }^{\circ}$ & 90 \\
\hline$V / \AA^{3}$ & $584.67(17)$ \\
\hline$Z$ & 4 \\
\hline$F(000)$ & 448.00 \\
\hline Crystallis. solvent & hexane, 1,2 -dichloroethane \\
\hline Colour of crystals & colourless \\
\hline Shape of crystals & block \\
\hline Radiation, $\lambda / \AA$ & Mo $K \alpha(\lambda=0.71069)$ \\
\hline $2 \Theta$ range $/^{\circ}$ & 6.139 to 57.158 \\
\hline Reflections collected & 2376 \\
\hline Data/restraints/parameters & $1389 / 0 / 73$ \\
\hline Goodness-of-fit on $F^{2}$ & 0.477 \\
\hline Final $R$ indexes [all data] & $R_{1}=0.0273, w R_{2}=0.0646$ \\
\hline
\end{tabular}


Single crystals of $\mathbf{8 b}$ were obtained by recrystallization from hexane/dichloromethane by vapor diffusion. Single crystal X-ray diffraction analyses were conducted using Bruker Apex-II

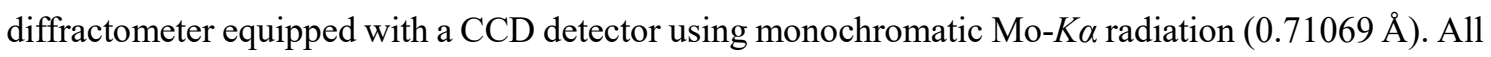
non-hydrogen atoms were refined with anisotropic atomic displacement parameters. Hydrogen atoms attached to carbon atoms were treated as riding atoms, using isotropic displacement parameters.

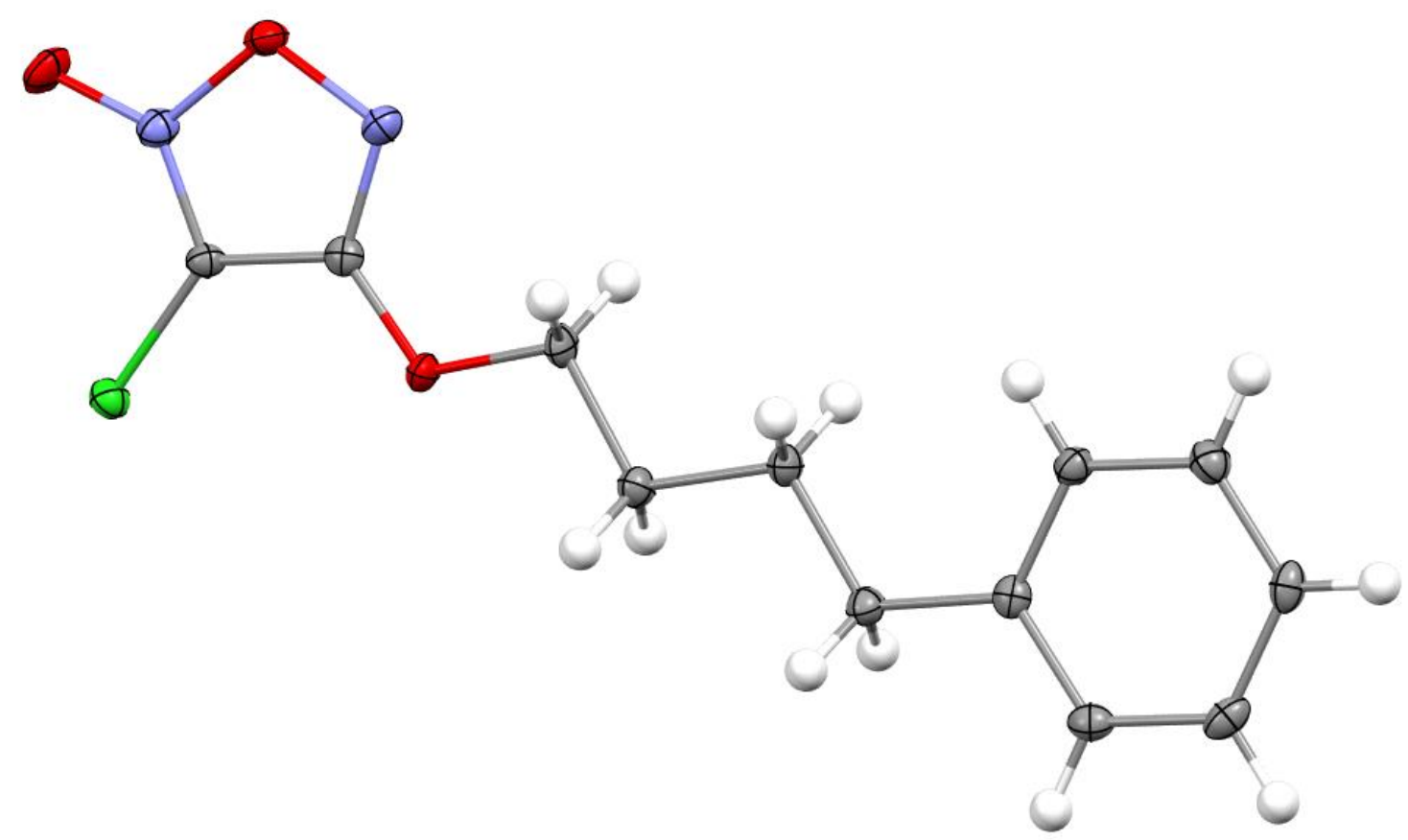

Asymmetric unit in the crystal structure of $\mathbf{8 b}$, thermal ellipsoids are drawn at 50\% probability level. Hydrogen atoms are drawn as fixed-size spheres of radius $0.20 \AA$.

Table S2. Crystal data and structure refinement details.

\begin{tabular}{|l|l|}
\hline Empirical formula & $\mathrm{C}_{12} \mathrm{H}_{13} \mathrm{ClN}_{2} \mathrm{O}_{3}$ \\
\hline Formula weight & 268.70 \\
\hline Temperature/K & 90 \\
\hline Crystal system & orthorhombic \\
\hline Space group & $P 2_{1}$ \\
\hline$a / \AA$ & $7.5174(6)$ \\
\hline$b / \AA$ & $10.8721(9)$ \\
\hline$c / \AA$ & $7.6231(6)$ \\
\hline$\alpha /^{\circ}$ & 90 \\
\hline$\beta /^{\circ}$ & $92.3519(10)$ \\
\hline$\gamma /^{\circ}$ & 90 \\
\hline
\end{tabular}




\begin{tabular}{|l|l|}
\hline$V / \AA^{3}$ & $622.51(9)$ \\
\hline$Z$ & 2 \\
\hline$F(000)$ & 280.00 \\
\hline Crystallis. solvent & hexane, dichloromethane \\
\hline Colour of crystals & colourless \\
\hline Shape of crystals & block \\
\hline Radiation, $\lambda / \AA$ & Mo $K \alpha(\lambda=0.71069)$ \\
\hline $2 \Theta$ range $/{ }^{\circ}$ & 5.424 to 57.47 \\
\hline Reflections collected & 2942 \\
\hline Data/restraints/parameters & $2190 / 1 / 163$ \\
\hline Goodness-of-fit on $F^{2}$ & 1.055 \\
\hline Final $R$ indexes [all data] & $R_{1}=0.0227, w R_{2}=0.0612$ \\
\hline
\end{tabular}


Single crystals of $\mathbf{8 f}$ were obtained by recrystallization from hexane/ethyl acetate by vapor diffusion. Single crystal X-ray diffraction analyses were conducted using Bruker Apex-II diffractometer equipped with a CCD detector using monochromatic Mo- $K \alpha$ radiation ( $0.71069 \AA)$. All non-hydrogen atoms were refined with anisotropic atomic displacement parameters. Hydrogen atoms attached to carbon atoms were treated as riding atoms, using isotropic displacement parameters.

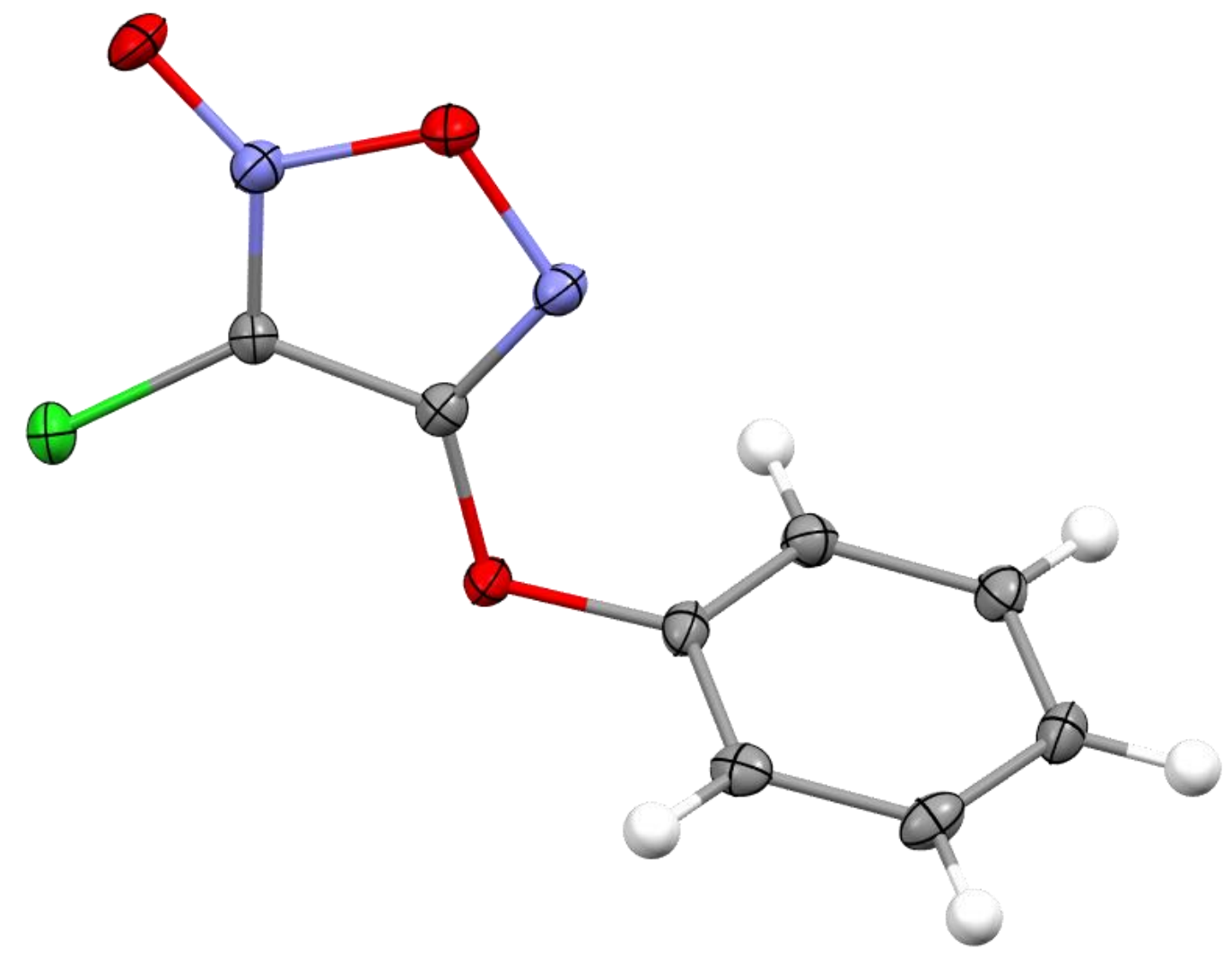

Asymmetric unit in the crystal structure of $\mathbf{8 f}$, thermal ellipsoids are drawn at 50\% probability level. Hydrogen atoms are drawn as fixed-size spheres of radius $0.20 \AA$.

Table S3. Crystal data and structure refinement details.

\begin{tabular}{|l|l|}
\hline Empirical formula & $\mathrm{C}_{8} \mathrm{H}_{5} \mathrm{ClN}_{2} \mathrm{O}_{3}$ \\
\hline Formula weight & 212.59 \\
\hline Temperature/K & 90 \\
\hline Crystal system & orthorhombic \\
\hline Space group & $P 2_{1}$ \\
\hline$a / \AA$ & $6.5586(13)$ \\
\hline$b / \AA$ & $7.4628(14)$ \\
\hline$c / \AA$ & $8.5758(17)$ \\
\hline$\alpha /^{\circ}$ & 90 \\
\hline$\beta /^{\circ}$ & 90 \\
\hline
\end{tabular}




\begin{tabular}{|l|l|}
\hline$\gamma /{ }^{\circ}$ & 90 \\
\hline$V / \AA^{3}$ & $419.48(14)$ \\
\hline$Z$ & 2 \\
\hline$F(000)$ & 216 \\
\hline Crystallis. solvent & hexane, acetone \\
\hline Colour of crystals & colourless \\
\hline Shape of crystals & block \\
\hline Radiation, $\lambda / \AA$ & Mo $K \alpha(\lambda=0.71069)$ \\
\hline $2 \Theta$ range $/{ }^{\circ}$ & 4.751 to 54.957 \\
\hline Reflections collected & 1212 \\
\hline Data/restraints/parameters & $1663 / 1 / 127$ \\
\hline Goodness-of-fit on $F^{2}$ & 1.030 \\
\hline Final $R$ indexes [all data] & $R_{1}=0.0312, w R_{2}=0.0760$ \\
\hline
\end{tabular}


Single crystals of 11a were obtained by recrystallization from hexane/1,2-dichloroethane by vapor diffusion. Single crystal X-ray diffraction analyses were conducted using Bruker Apex-II

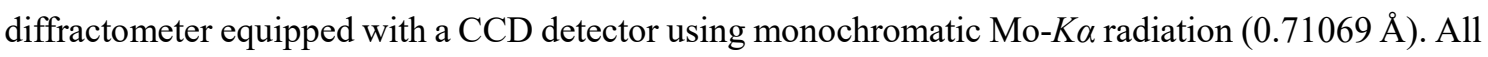
non-hydrogen atoms were refined with anisotropic atomic displacement parameters. Hydrogen atoms attached to carbon atoms were treated as riding atoms, using isotropic displacement parameters.

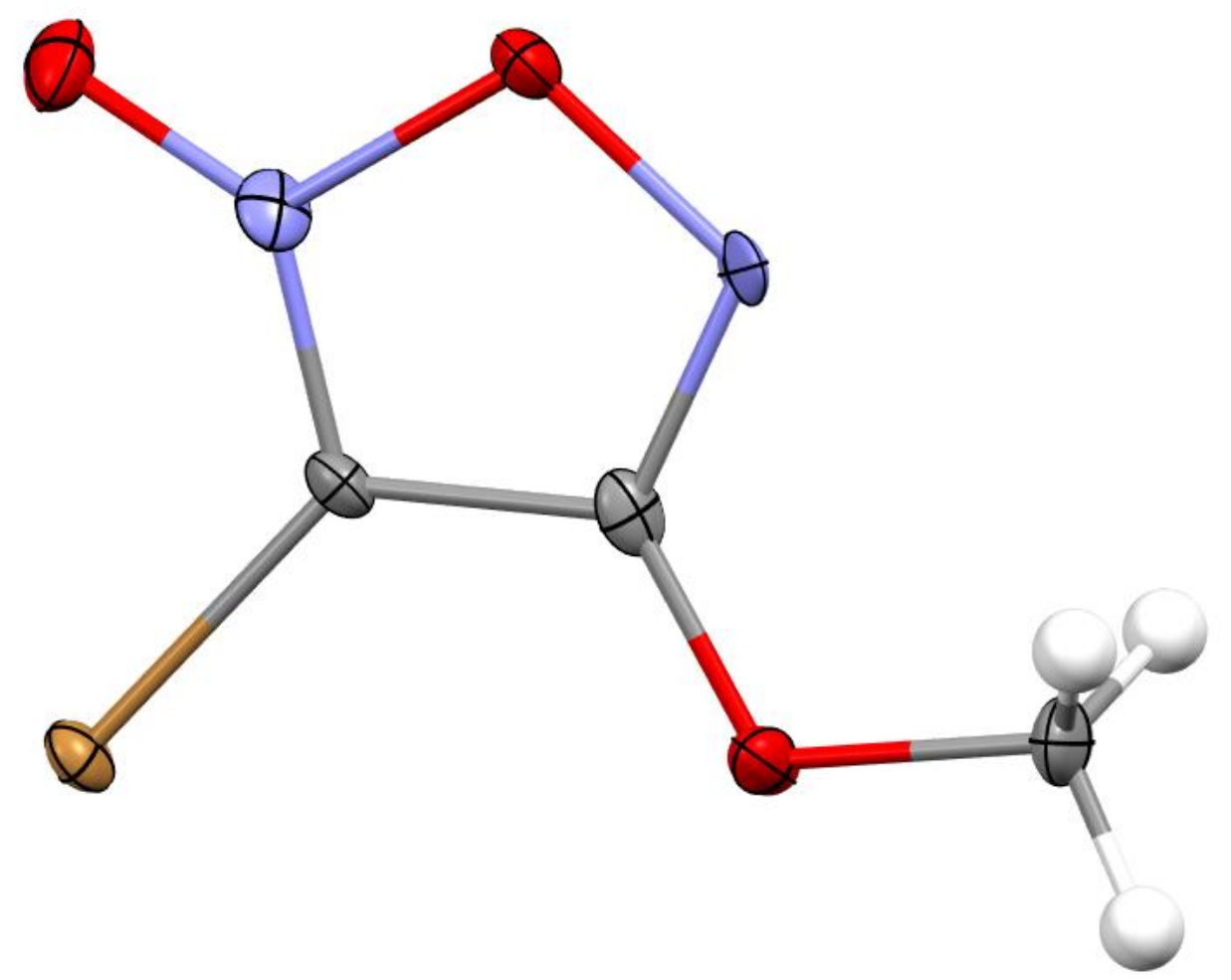

Asymmetric unit in the crystal structure of $11 \mathrm{a}$, thermal ellipsoids are drawn at 50\% probability level. Hydrogen atoms are drawn as fixed-size spheres of radius $0.20 \AA$.

Table S4. Crystal data and structure refinement details.

\begin{tabular}{|l|l|}
\hline Empirical formula & $\mathrm{C}_{3} \mathrm{H}_{3} \mathrm{BrN}_{2} \mathrm{O}_{3}$ \\
\hline Formula weight & 194.97 \\
\hline Temperature/K & 90 \\
\hline Crystal system & orthorhombic \\
\hline Space group & $P 2_{1}$ \\
\hline$a / \AA$ & $6.849(6)$ \\
\hline$b / \AA$ & $6.272(5)$ \\
\hline$c / \AA$ & $7.042(6)$ \\
\hline$\alpha /{ }^{\circ}$ & 90 \\
\hline
\end{tabular}




\begin{tabular}{|l|l|}
\hline$\beta /{ }^{\circ}$ & $106.901(10)$ \\
\hline$\gamma{ }^{\circ}$ & 90 \\
\hline$V / \AA^{3}$ & $289.4(4)$ \\
\hline$Z$ & 2 \\
\hline$F(000)$ & 188.00 \\
\hline Crystallis. solvent & hexane, 1,2 -dichloroethane \\
\hline Colour of crystals & colourless \\
\hline Shape of crystals & block \\
\hline Radiation, $\lambda / \AA$ & Mo $K \alpha(\lambda=0.71069)$ \\
\hline $2 \Theta$ range $/{ }^{\circ}$ & 6.215 to 57.164 \\
\hline Reflections collected & 1360 \\
\hline Data/restraints/parameters & $1113 / 1 / 82$ \\
\hline Goodness-of-fit on $F^{2}$ & 1.117 \\
\hline Final $R$ indexes [all data] & $R_{1}=0.0255, w R_{2}=0.0673$ \\
\hline
\end{tabular}


Single crystals of 15 were obtained by recrystallization from hexane/1,2-dichloroethane by vapor diffusion. Single crystal X-ray diffraction analyses were conducted using Bruker Apex-II

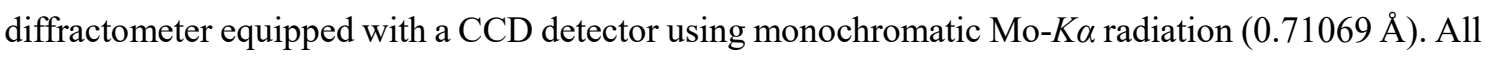
non-hydrogen atoms were refined with anisotropic atomic displacement parameters. Hydrogen atoms attached to carbon atoms were treated as riding atoms, using isotropic displacement parameters.

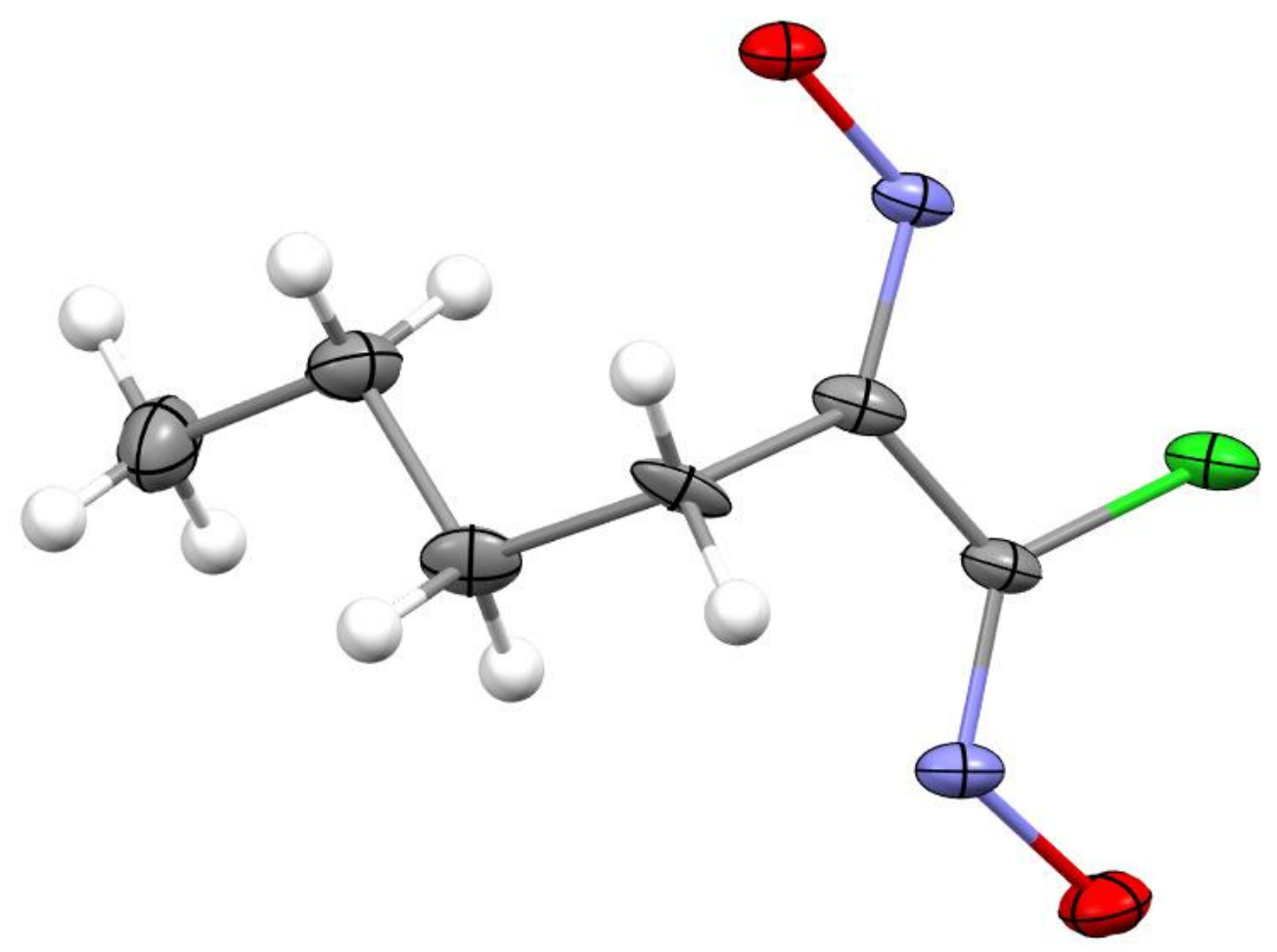

Asymmetric unit in the crystal structure of $\mathbf{1 5}$, thermal ellipsoids are drawn at 50\% probability level. Hydrogen atoms are drawn as fixed-size spheres of radius $0.20 \AA$.

Table S5. Crystal data and structure refinement details.

\begin{tabular}{|l|l|}
\hline Empirical formula & $\mathrm{C}_{6} \mathrm{H}_{11} \mathrm{ClN}_{2} \mathrm{O}_{2}$ \\
\hline Formula weight & 178.62 \\
\hline Temperature/K & 90 \\
\hline Crystal system & orthorhombic \\
\hline Space group & $P-1$ \\
\hline$a / \AA$ & $5.360(7)$ \\
\hline$b / \AA$ & $9.166(14)$ \\
\hline$c / \AA$ & $18.17(3)$ \\
\hline$\alpha /^{\circ}$ & $94.47(3)$ \\
\hline$\beta /^{\circ}$ & $94.802(14)$ \\
\hline
\end{tabular}




\begin{tabular}{|l|l|}
\hline$\gamma /{ }^{\circ}$ & $101.150(16)$ \\
\hline$V / \AA^{3}$ & $869(2)$ \\
\hline$Z$ & 4 \\
\hline$F(000)$ & 376.00 \\
\hline Crystallis. solvent & hexane, 1,2 -dichloroethane \\
\hline Colour of crystals & colourless \\
\hline Shape of crystals & block \\
\hline Radiation, $\lambda / \AA$ & Mo $K \alpha(\lambda=0.71069)$ \\
\hline $2 \Theta$ range $/{ }^{\circ}$ & 4.521 to 52.912 \\
\hline Reflections collected & 1510 \\
\hline Data/restraints/parameters & $3023 / 0 / 199$ \\
\hline Goodness-of-fit on $F^{2}$ & 1.082 \\
\hline Final $R$ indexes [all data] & $R_{1}=0.1346, w R_{2}=0.4165$ \\
\hline
\end{tabular}


Single crystals of $\mathbf{1 9 b}$ were obtained by recrystallization from hexane/1,2-dichloroethane by vapor diffusion. Single crystal X-ray diffraction analyses were conducted using Bruker Apex-II

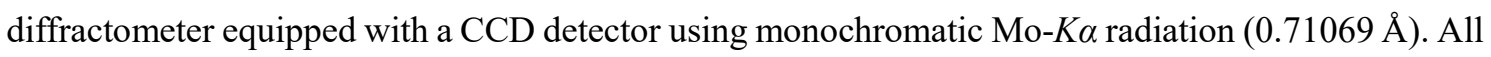
non-hydrogen atoms were refined with anisotropic atomic displacement parameters. Hydrogen atoms attached to carbon atoms were treated as riding atoms, using isotropic displacement parameters. Because the crystals with high quality were difficult to obtain, several A level alerts could not be corrected. We left this issue because the structure of $\mathbf{1 9 b}$ was unambiguously determined by the other analytical methods and the XRD data are just complementary.

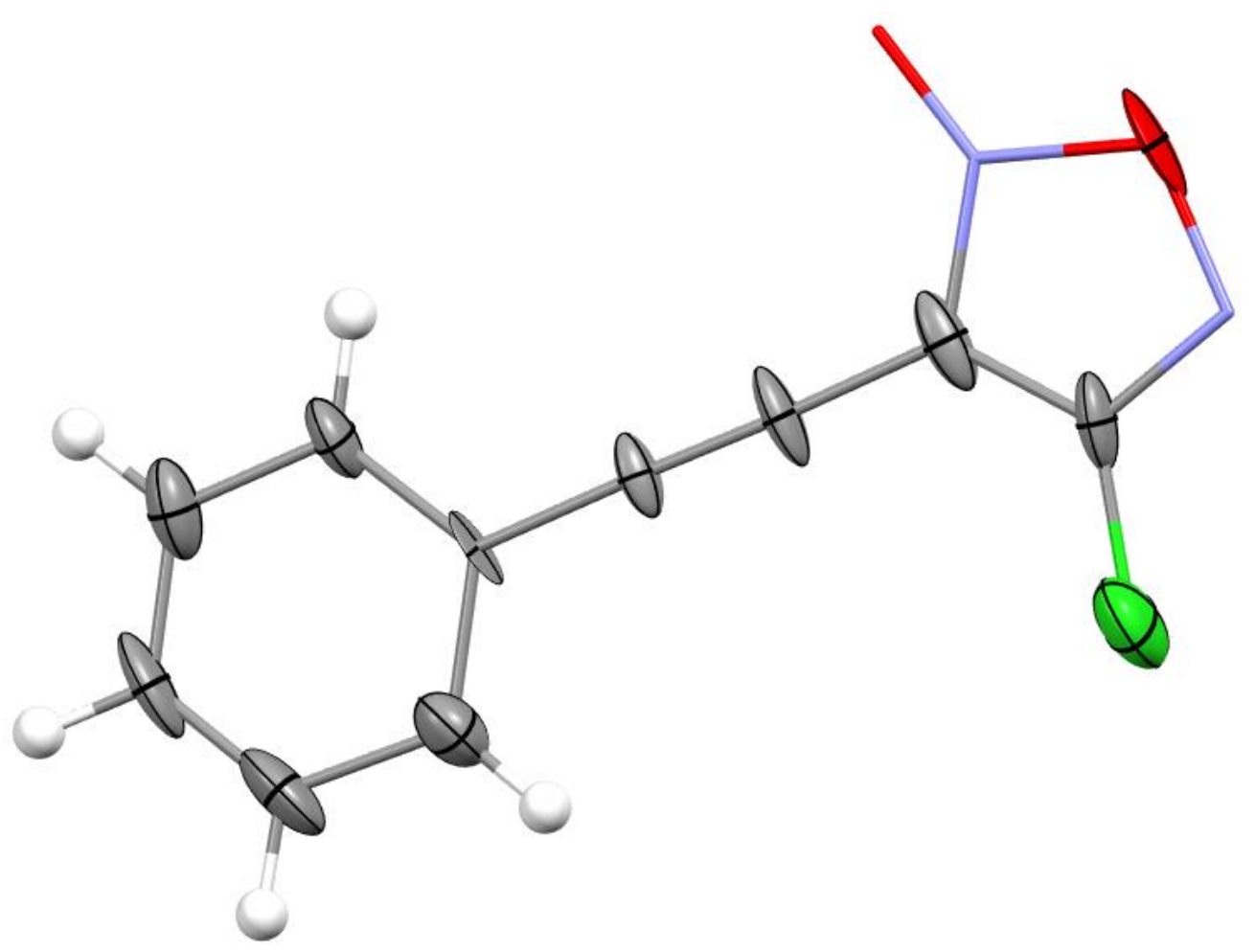

Asymmetric unit in the crystal structure of $\mathbf{1 9 b}$, thermal ellipsoids are drawn at $50 \%$ probability level. Hydrogen atoms are drawn as fixed-size spheres of radius $0.20 \AA$.

Table S6. Crystal data and structure refinement details.

\begin{tabular}{|l|l|}
\hline Empirical formula & $\mathrm{C}_{10} \mathrm{H}_{5} \mathrm{ClN}_{2} \mathrm{O}_{2}$ \\
\hline Formula weight & 220.61 \\
\hline Temperature/K & 90 \\
\hline Crystal system & orthorhombic \\
\hline Space group & $P-1$ \\
\hline$a / \AA$ & $6.6670(13)$ \\
\hline$b / \AA$ & $6.7899(14)$ \\
\hline
\end{tabular}




\begin{tabular}{|l|l|}
\hline$c / \AA$ & $11.050(2)$ \\
\hline$\alpha{ }^{\circ}$ & $81.39(3)$ \\
\hline$\beta /{ }^{\circ}$ & $86.15(3)$ \\
\hline$\gamma{ }^{\circ}$ & $75.78(3)$ \\
\hline$V / \AA^{3}$ & $479.18(18)$ \\
\hline$Z$ & 2 \\
\hline$F(000)$ & 224 \\
\hline Crystallis. solvent & hexane, 1,2 -dichloroethane \\
\hline Colour of crystals & colourless \\
\hline Shape of crystals & block \\
\hline Radiation, $\lambda / \AA$ & Mo $K \alpha(\lambda=0.71069)$ \\
\hline $2 \Theta$ range ${ }^{\circ}$ & 6.280 to 50.392 \\
\hline Reflections collected & 479 \\
\hline Data/restraints/parameters & $1548 / 186 / 136$ \\
\hline Goodness-of-fit on $F^{2}$ & 1.550 \\
\hline Final $R$ indexes [all data] & $R_{1}=0.1618, w R_{2}=0.4356$ \\
\hline
\end{tabular}


Single crystals of $\mathbf{2 1 b}$ were obtained by recrystallization from hexane/ethyl acetate by vapor diffusion. Single crystal X-ray diffraction analyses were conducted using Bruker Apex-II diffractometer equipped with a CCD detector using monochromatic Mo- $K \alpha$ radiation (0.71069 $\AA$ ). All non-hydrogen atoms were refined with anisotropic atomic displacement parameters. Hydrogen atoms attached to carbon atoms were treated as riding atoms, using isotropic displacement parameters.

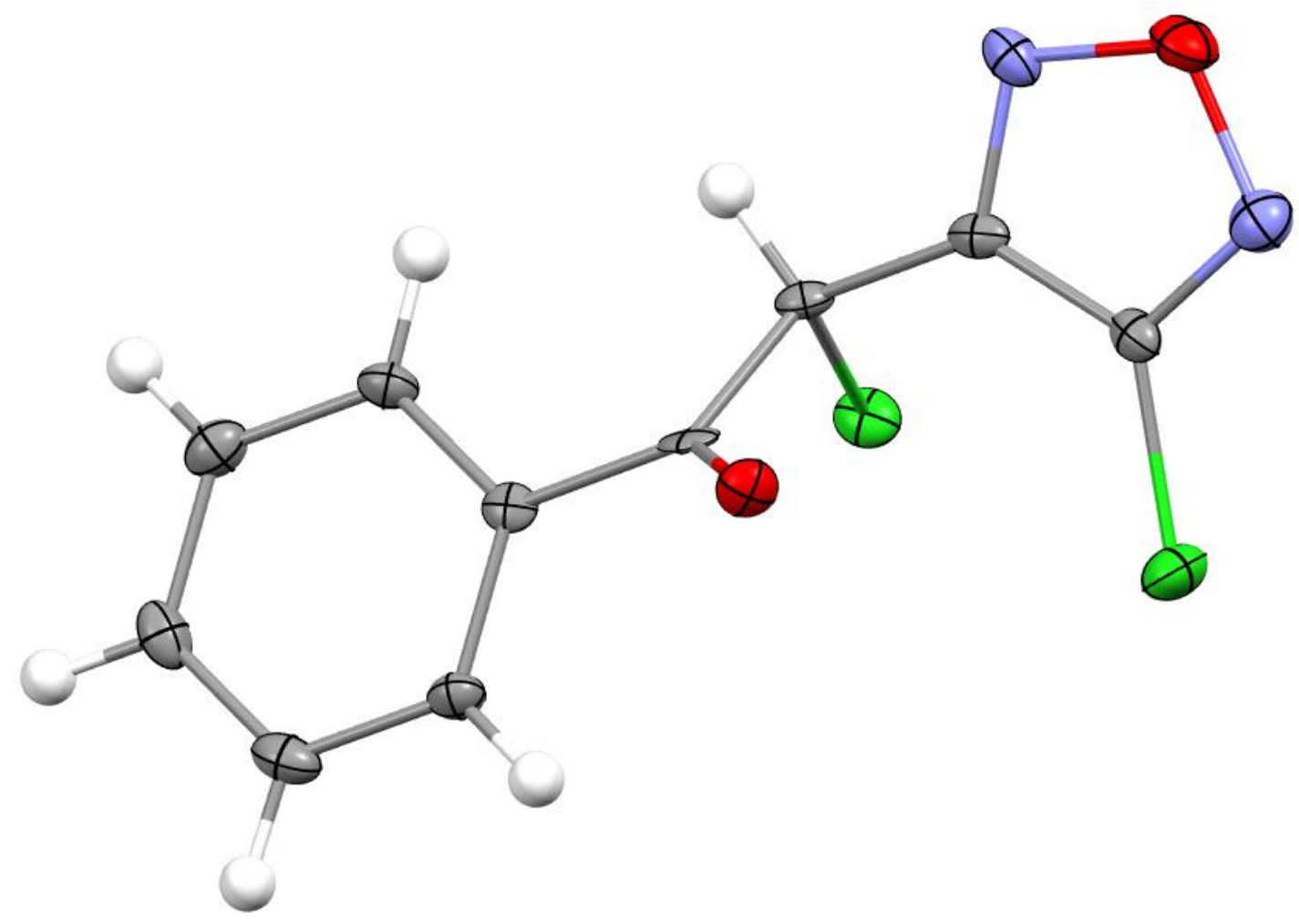

Asymmetric unit in the crystal structure of $\mathbf{2 1 b}$, thermal ellipsoids are drawn at 50\% probability level. Hydrogen atoms are drawn as fixed-size spheres of radius $0.20 \AA$.

Table S7. Crystal data and structure refinement details.

\begin{tabular}{|l|l|}
\hline Empirical formula & $\mathrm{C}_{10} \mathrm{H}_{6} \mathrm{Cl}_{2} \mathrm{~N}_{2} \mathrm{O}_{2}$ \\
\hline Formula weight & 257.08 \\
\hline Temperature/K & 90 \\
\hline Crystal system & orthorhombic \\
\hline Space group & $P-1$ \\
\hline$a / \AA$ & $5.5643(19)$ \\
\hline$b / \AA$ & $9.865(4)$ \\
\hline$c / \AA$ & $10.798(4)$ \\
\hline$\alpha /{ }^{\circ}$ & $112.164(5)$ \\
\hline
\end{tabular}




\begin{tabular}{|l|l|}
\hline$\beta /{ }^{\circ}$ & $95.909(6)$ \\
\hline$\gamma{ }^{\circ}$ & $103.111(5)$ \\
\hline$V / \AA^{3}$ & $522.8(4)$ \\
\hline$Z$ & 2 \\
\hline$F(000)$ & 260.00 \\
\hline Crystallis. solvent & hexane, ethyl acetate \\
\hline Colour of crystals & colourless \\
\hline Shape of crystals & block \\
\hline Radiation, $\lambda / \AA$ & Mo $K \alpha(\lambda=0.71069)$ \\
\hline $2 \Theta$ range $/{ }^{\circ}$ & 4.656 to 55.180 \\
\hline Reflections collected & 802 \\
\hline Data/restraints/parameters & $1590 / 0 / 153$ \\
\hline Goodness-of-fit on $F^{2}$ & 1.042 \\
\hline Final $R$ indexes [all data] & $R_{1}=0.0373, w R_{2}=0.0889$ \\
\hline
\end{tabular}


Single crystals of 25a were obtained by recrystallization from hexane/ethyl acetate by vapor diffusion. Single crystal X-ray diffraction analyses were conducted using Bruker Apex-II diffractometer equipped with a CCD detector using monochromatic Mo- $K \alpha$ radiation ( $0.71069 \AA)$. All non-hydrogen atoms were refined with anisotropic atomic displacement parameters. Hydrogen atoms attached to carbon atoms were treated as riding atoms, using isotropic displacement parameters.

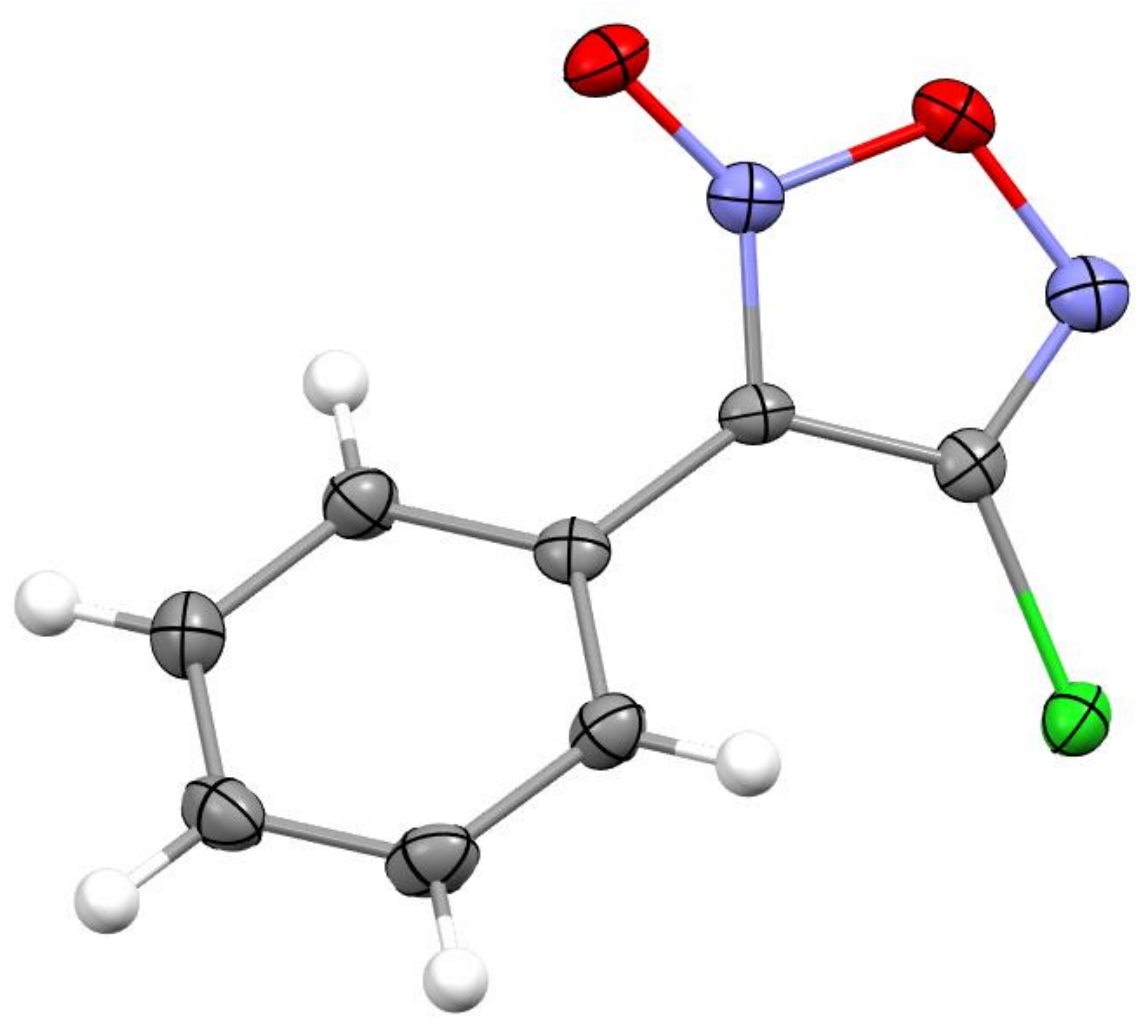

Asymmetric unit in the crystal structure of 25a, thermal ellipsoids are drawn at 50\% probability level. Hydrogen atoms are drawn as fixed-size spheres of radius $0.20 \AA$.

Table S8. Crystal data and structure refinement details.

\begin{tabular}{|l|l|}
\hline Empirical formula & $\mathrm{C}_{64} \mathrm{H}_{40} \mathrm{Cl}_{8} \mathrm{~N}_{16} \mathrm{O}_{16}$ \\
\hline Formula weight & 1572.74 \\
\hline Temperature/K & 90 \\
\hline Crystal system & orthorhombic \\
\hline Space group & $P$ b c a \\
\hline$a / \AA$ & $12.5251(19)$ \\
\hline$b / \AA$ & $7.7888(12)$ \\
\hline$c / \AA$ & $16.980(3)$ \\
\hline$\alpha /{ }^{\circ}$ & 90 \\
\hline
\end{tabular}




\begin{tabular}{|l|l|}
\hline$\beta /^{\circ}$ & 90 \\
\hline$\gamma{ }^{\circ}$ & 90 \\
\hline$V / \AA^{3}$ & $1656.5(5)$ \\
\hline$Z$ & 1 \\
\hline$F(000)$ & 800.00 \\
\hline Crystallis. solvent & hexane, ethyl acetate \\
\hline Colour of crystals & colourless \\
\hline Shape of crystals & block \\
\hline Radiation, $\lambda / \AA$ & Mo $K \alpha(\lambda=0.71069)$ \\
\hline $2 \Theta$ range $/{ }^{\circ}$ & 5.797 to 55.270 \\
\hline Reflections collected & 1842 \\
\hline Data/restraints/parameters & $1889 / 0 / 118$ \\
\hline Goodness-of-fit on $F^{2}$ & 1.012 \\
\hline Final $R$ indexes [all data] & $R_{1}=0.0365, w R_{2}=0.0908$ \\
\hline
\end{tabular}


Single crystals of $27 \mathbf{c}$ were obtained by recrystallization from hexane/1.2-dichloroethane by vapor diffusion. Single crystal X-ray diffraction analyses were conducted using Bruker Apex-II

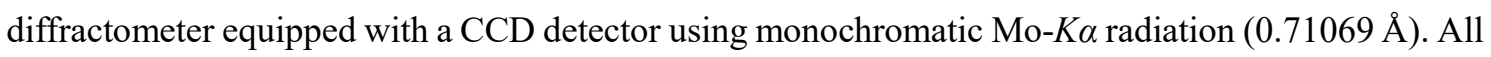
non-hydrogen atoms were refined with anisotropic atomic displacement parameters. Hydrogen atoms attached to carbon atoms were treated as riding atoms, using isotropic displacement parameters.

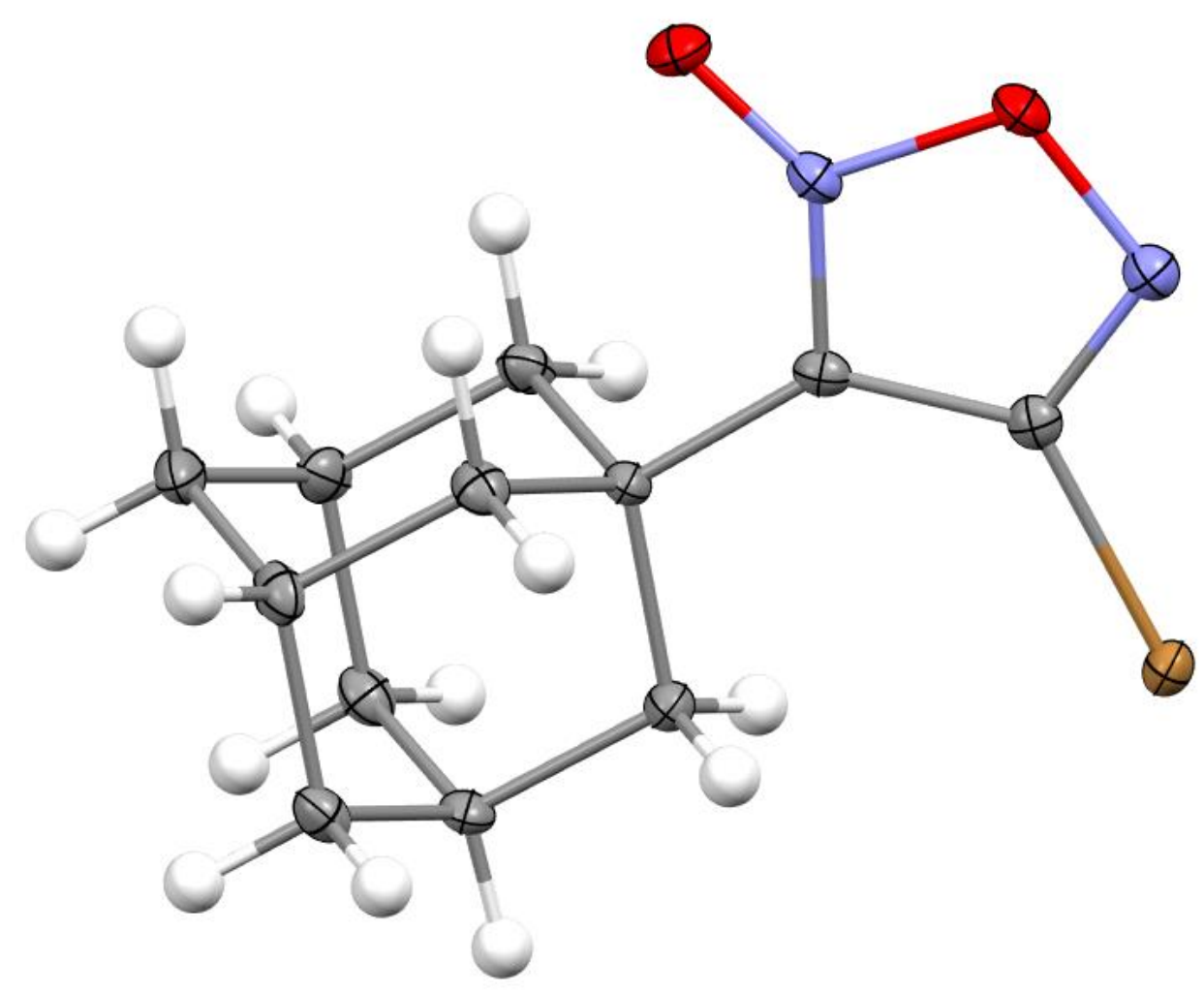

Asymmetric unit in the crystal structure of $\mathbf{2 7} \mathbf{c}$, thermal ellipsoids are drawn at 50\% probability level. Hydrogen atoms are drawn as fixed-size spheres of radius $0.20 \AA$.

Table S9. Crystal data and structure refinement details.

\begin{tabular}{|l|l|}
\hline Empirical formula & $\mathrm{C}_{12} \mathrm{H}_{15} \mathrm{BrN}_{2} \mathrm{O}_{2}$ \\
\hline Formula weight & 299.17 \\
\hline Temperature/K & 90 \\
\hline Crystal system & orthorhombic \\
\hline Space group & $P 2_{1} / \mathrm{m}$ \\
\hline$a / \AA$ & 9.2209 \\
\hline$b / \AA$ & 6.6668 \\
\hline$c / \AA$ & 9.3831 \\
\hline$\alpha /{ }^{\circ}$ & 90 \\
\hline
\end{tabular}




\begin{tabular}{|l|l|}
\hline$\beta /{ }^{\circ}$ & 99.2121 \\
\hline$\gamma{ }^{\circ}$ & 90 \\
\hline$V / \AA^{3}$ & 569.3762 \\
\hline$Z$ & 2 \\
\hline$F(000)$ & 304.00 \\
\hline Crystallis. solvent & hexane, 1.2 -dichloroethane \\
\hline Colour of crystals & colourless \\
\hline Shape of crystals & block \\
\hline Radiation, $\lambda / \AA$ & Mo $K \alpha(\lambda=0.71069)$ \\
\hline $2 \Theta$ range $/{ }^{\circ}$ & 5.755 to 57.405 \\
\hline Reflections collected & 2653 \\
\hline Data/restraints/parameters & $1137 / 0 / 128$ \\
\hline Goodness-of-fit on $F^{2}$ & 1.077 \\
\hline Final $R$ indexes [all data] & $R_{1}=0.0178, w R_{2}=0.0465$ \\
\hline
\end{tabular}


3. NMR spectra

$$
\begin{array}{|ll|}
\hline \multicolumn{1}{|c|}{\text { Parameters }} & \multicolumn{1}{c|}{\text { Values }} \\
\text { 1 Solvent } & \multicolumn{1}{c|}{\mathrm{CDCl}_{3}} \\
\text { 2 Temperature } & 294.7 \\
\text { 3 Number of Scans } & 165 \\
\text { 4 Spectrometer Frequency } 100.62 \\
\text { 5 Spectral Width } & 24038.5 \\
\text { 6 Lowest Frequency } & -1958.8 \\
\text { 7 Nucleus } & 13 \mathrm{C} \\
\text { 8 Spectral Size } & 65536 \\
\hline
\end{array}
$$<smiles>[O-][n+]1onc(Cl)c1Cl</smiles>

1
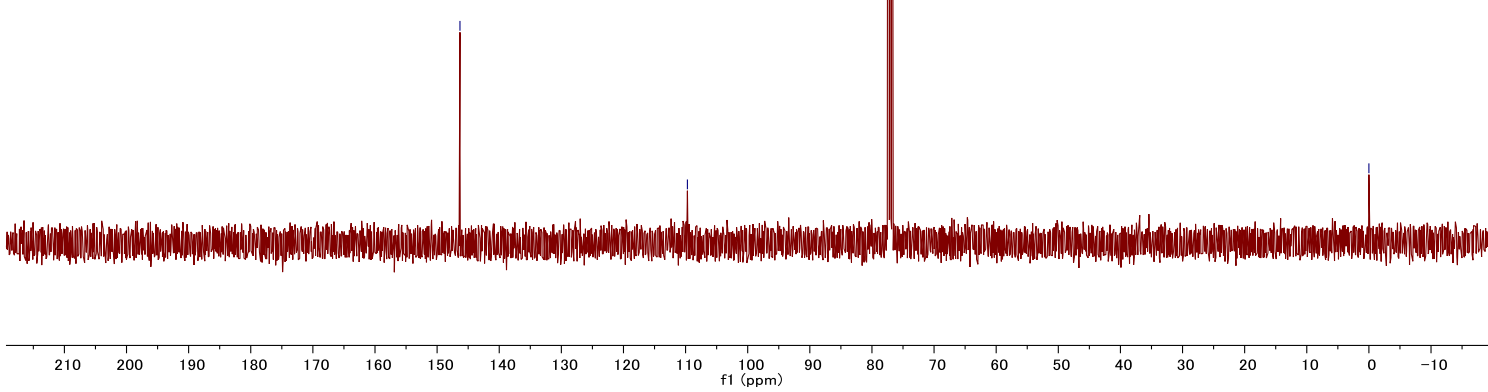


\begin{tabular}{|ll|}
\hline \multicolumn{1}{|c|}{ Parameters } & \multicolumn{1}{c|}{ Values } \\
1 Solvent & \multicolumn{1}{c|}{$\mathrm{CDCl} 3$} \\
2 Temperature & 298.4 \\
3 Number of Scans & 450 \\
4 Spectrometer Frequency 100.62 \\
5 Spectral Width & 24038.5 \\
6 Lowest Frequency & -1959.8 \\
7 Nucleus & $13 \mathrm{C}$ \\
8 Spectral Size & 65536 \\
\hline
\end{tabular}<smiles>[O-][n+]1onc(Br)c1Br</smiles>

2

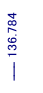

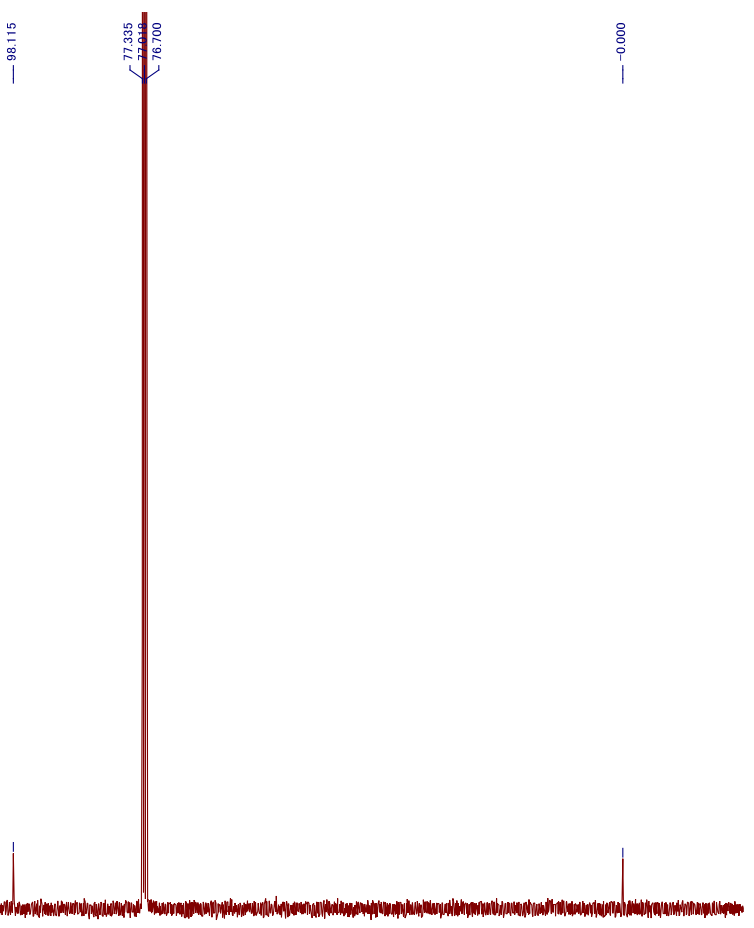

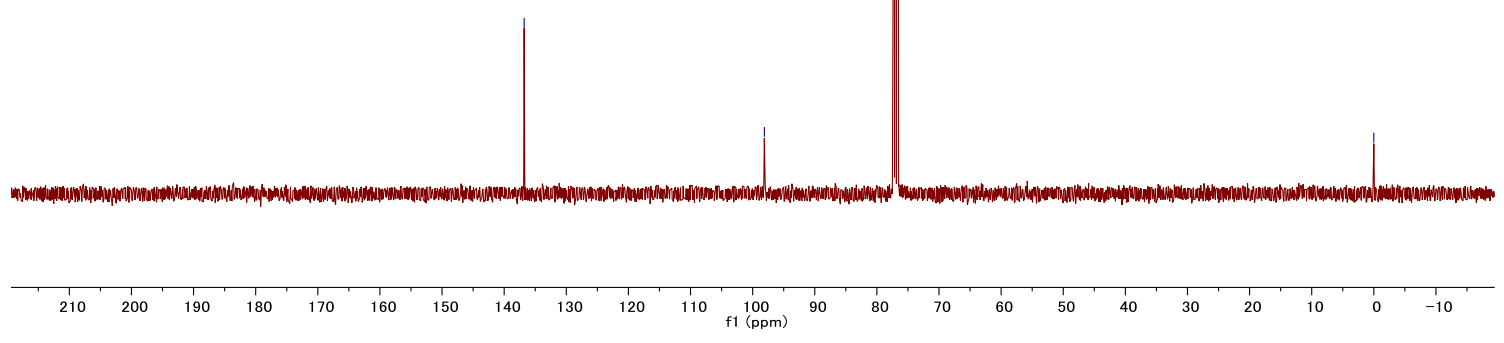

S34 


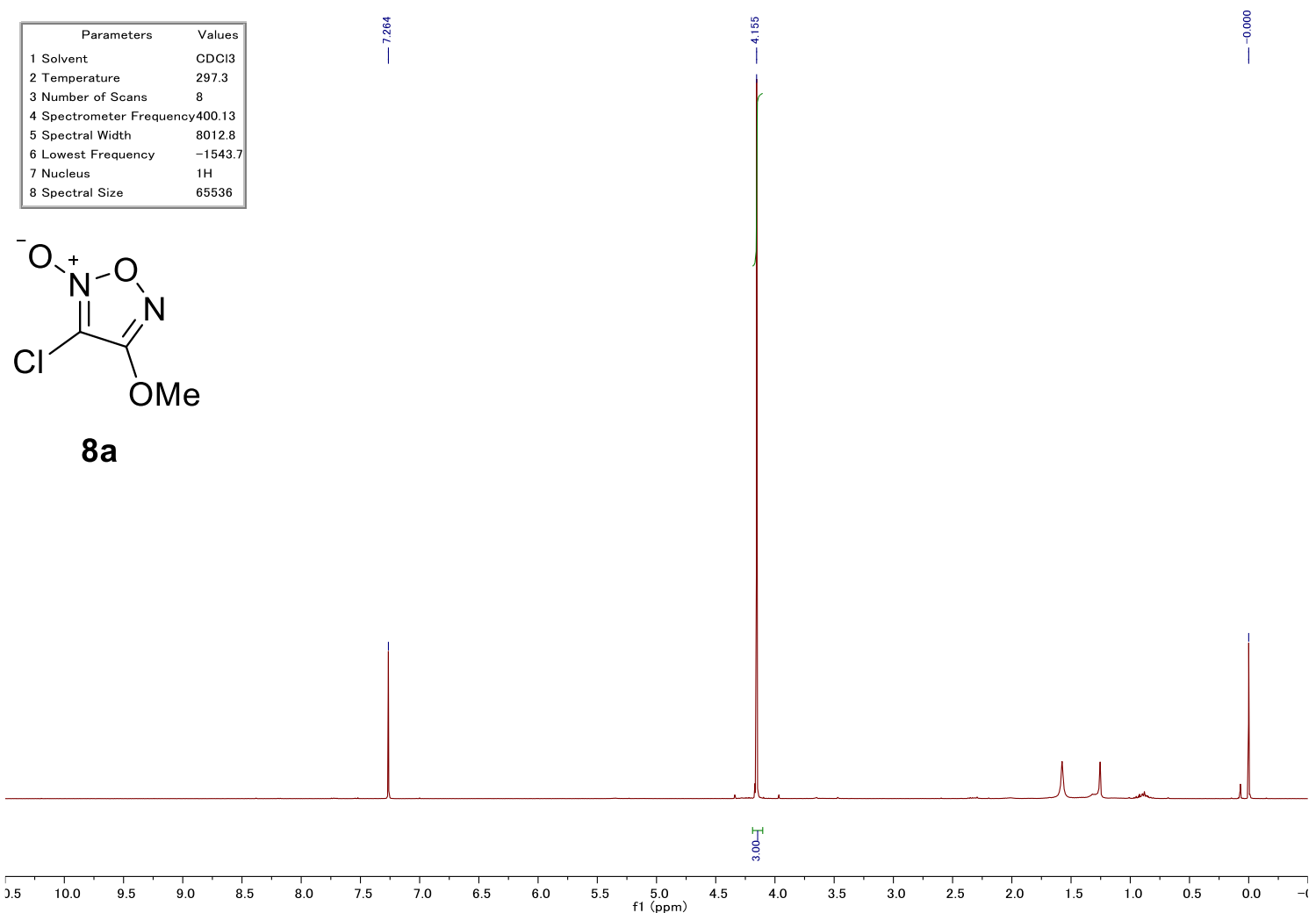

\begin{tabular}{|lc|}
\hline \multicolumn{1}{|c|}{ Parameters } & Values \\
1 Solvent & $\mathrm{CDCl3}$ \\
2 Temperature & 292.8 \\
3 Number of Scans & 1024 \\
4 Spectrometer Frequency 100.62 \\
5 Spectral Width & 24038.5 \\
6 Lowest Frequency & -1959.9 \\
7 Nucleus & $13 \mathrm{C}$ \\
8 Spectral Size & 65536 \\
\hline
\end{tabular}<smiles>COc1no[n+]([O-])c1Cl</smiles>

$8 a$

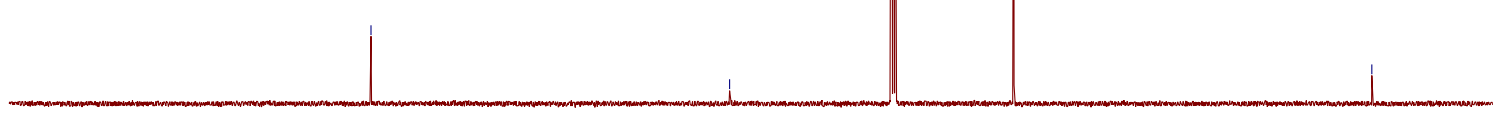

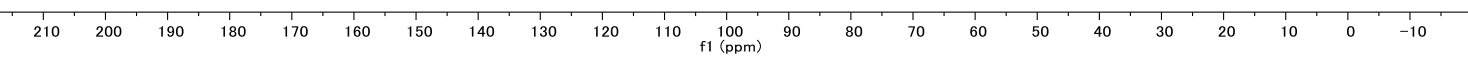



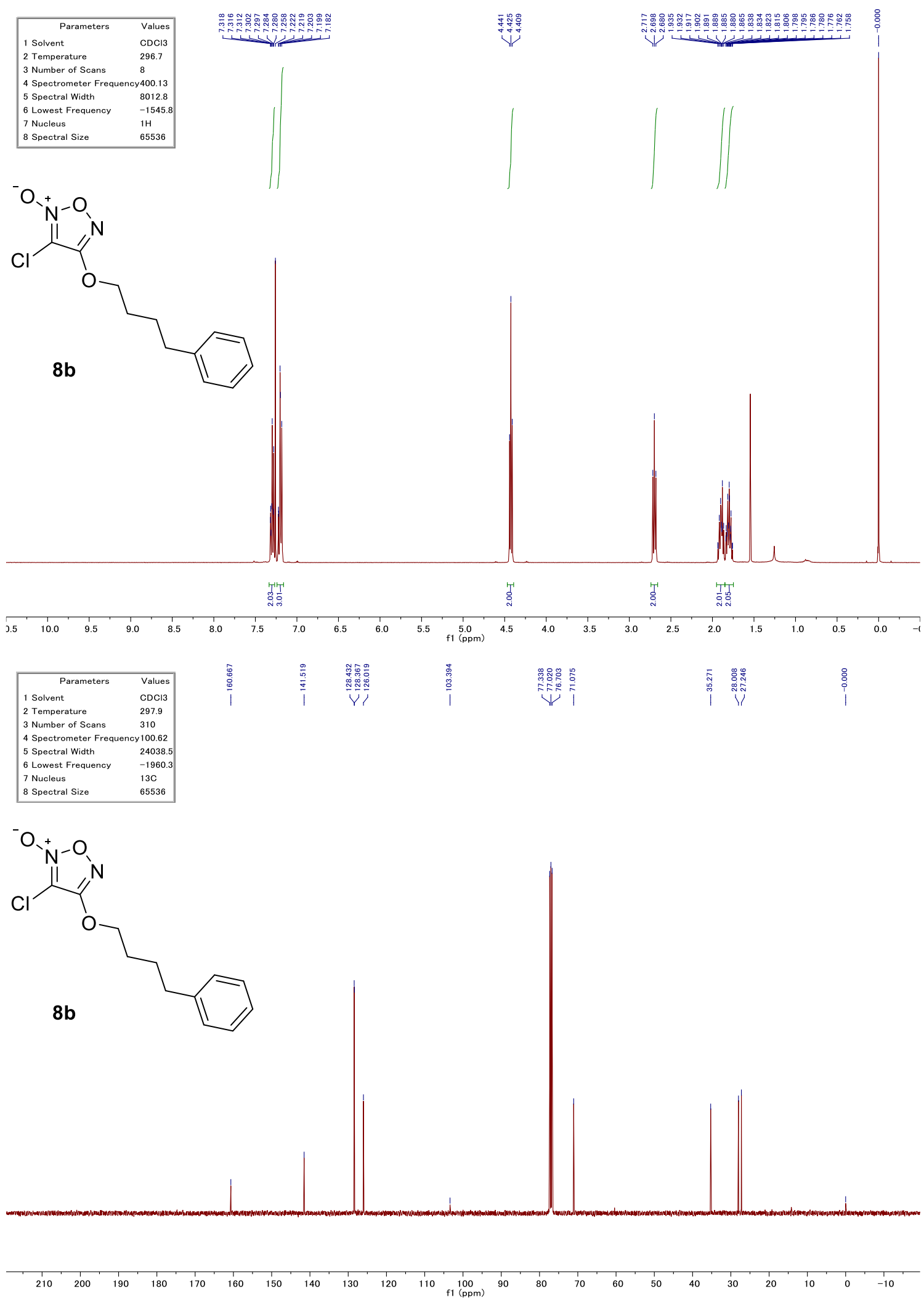


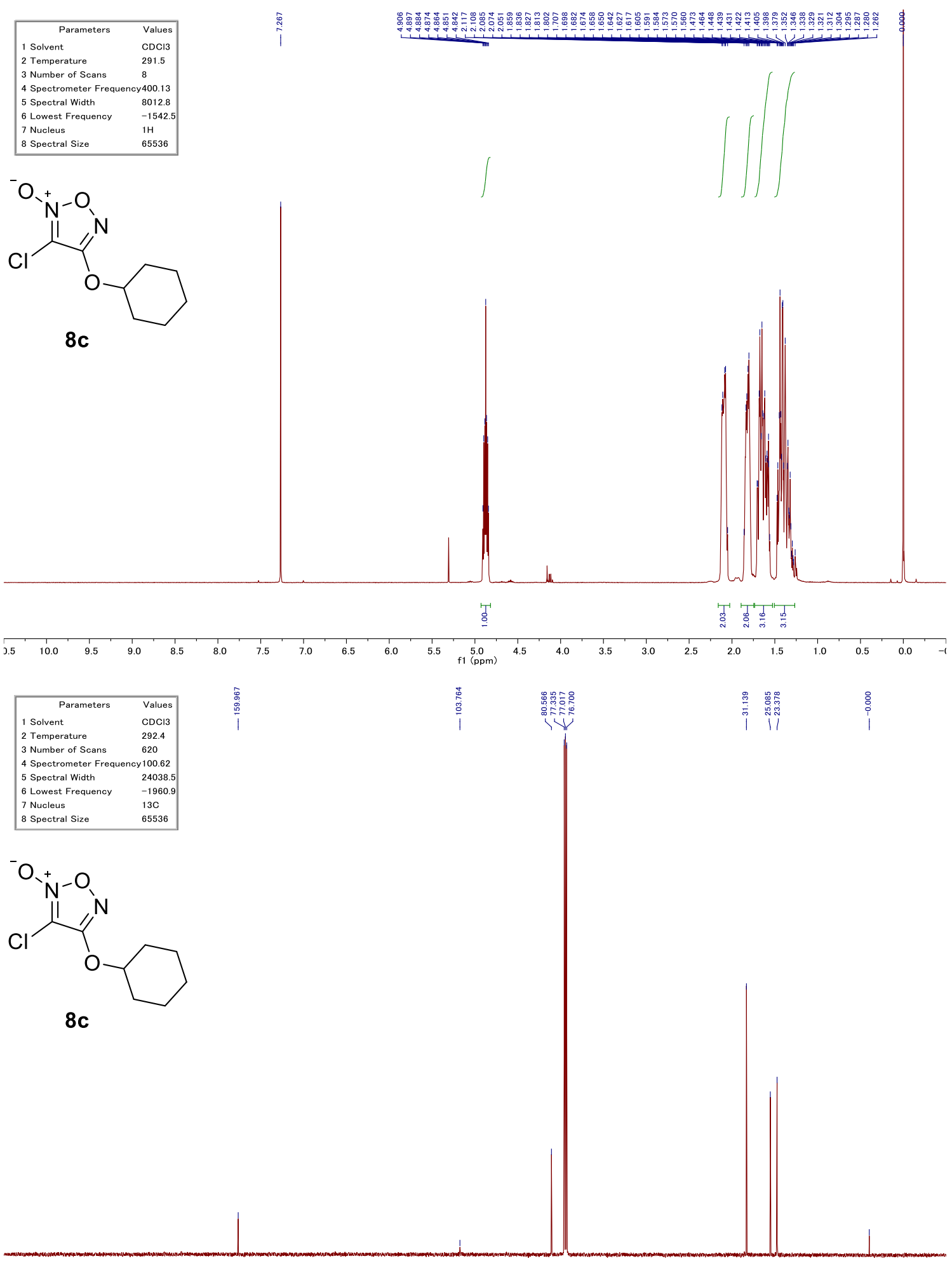

$\begin{array}{rllllllllllllllllllllll}1 & 1 \\ 210 & 200 & 190 & 180 & 170 & 160 & 150 & 140 & 130 & 120 & 110 & \underset{f 1}{100}(\mathrm{ppm}) & 90 & 80 & 70 & 60 & 50 & 40 & 30 & 20 & 10 & 0 & -10\end{array}$ 


\begin{tabular}{|lc|}
\hline \multicolumn{1}{|c|}{ Parameters } & Values \\
1 Solvent & CDCl13 \\
2 Temperature & 292.8 \\
3 Number of Scans & 8 \\
4 Spectrometer Frequency400.13 \\
5 Spectral Width & 8012.8 \\
6 Lowest Frequency & -1542. \\
7 Nucleus & $1 \mathrm{H}$ \\
8 Spectral Size & 65536 \\
\hline
\end{tabular}

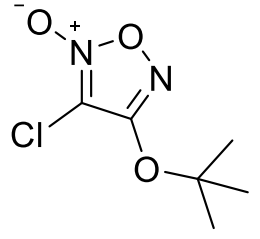

$8 d$

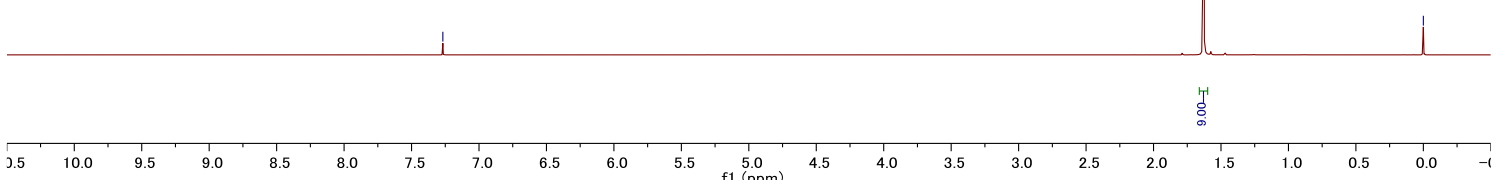

\begin{tabular}{|lc|}
\hline \multicolumn{1}{|c|}{ Parameters } & Values \\
1 Solvent & $\mathrm{CDCl3}$ \\
2 Temperature & 293.7 \\
3 Number of Scans & 1024 \\
4 Spectrometer Frequency 100.62 \\
5 Spectral Width & 24038.5 \\
6 Lowest Frequency & -1960.2 \\
7 Nucleus & $13 \mathrm{C}$ \\
8 Spectral Size & 65536 \\
\hline
\end{tabular}

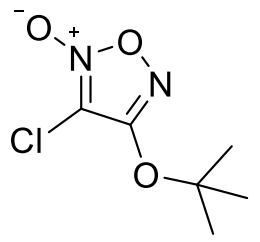

8d
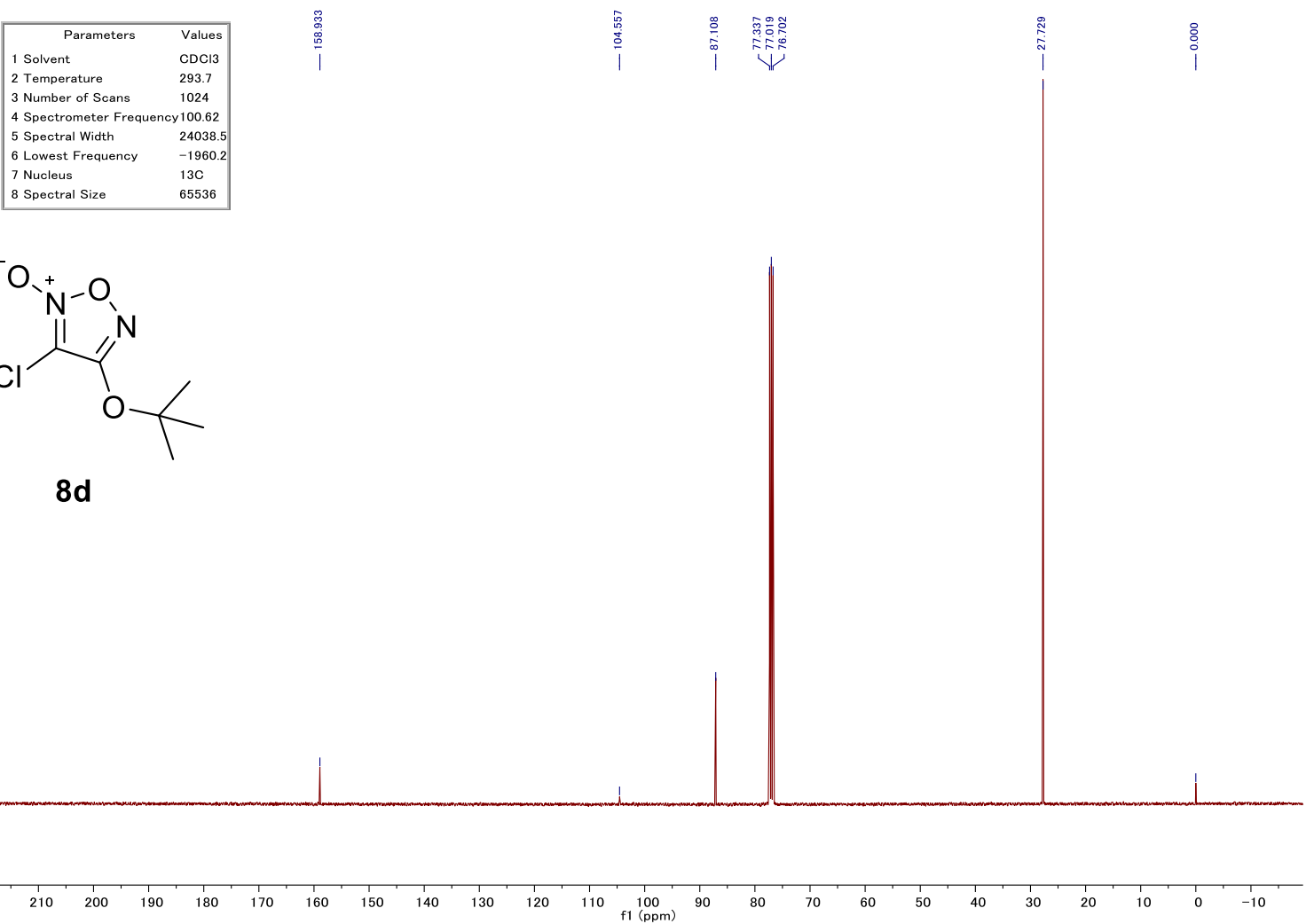

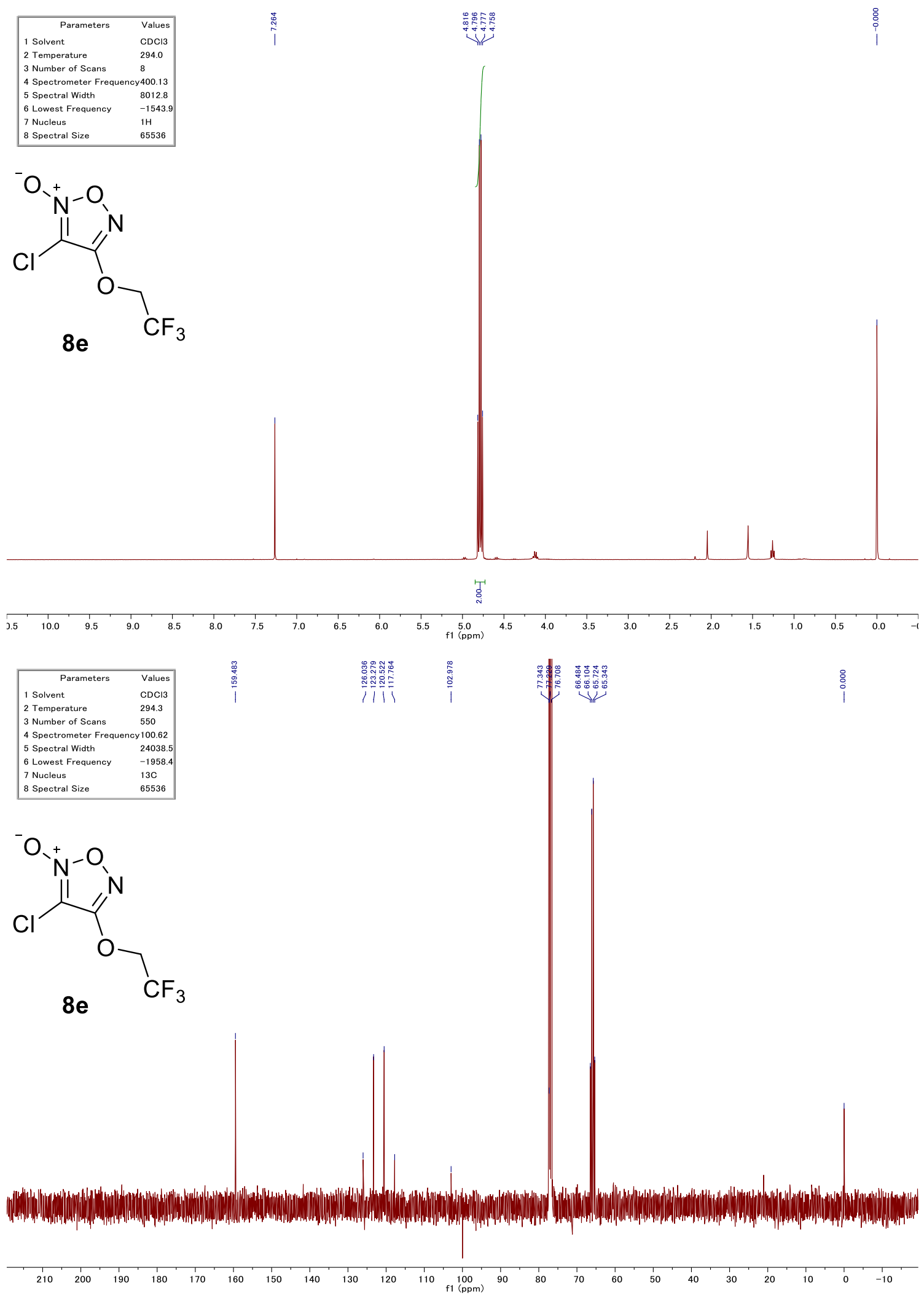


$$
\begin{array}{|ll|}
\hline \multicolumn{1}{|c|}{\text { Parameters }} & \multicolumn{1}{c|}{\text { Values }} \\
\text { 1 Solvent } & \text { CDC13 } \\
\text { 2 Temperature } & 292.9 \\
\text { 3 Number of Scans } & 16 \\
\text { 4 Spectrometer Frequency376.46 } \\
\text { 5 Spectral Width } & 300000.0 \\
\text { 6 Lowest Frequency } & -187036.8 \\
\text { 7 Nucleus } & 19 F \\
\text { 8 Spectral Size } & 131072 \\
\hline
\end{array}
$$

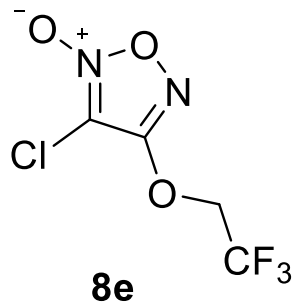

$$
3
$$

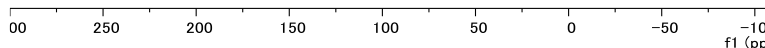



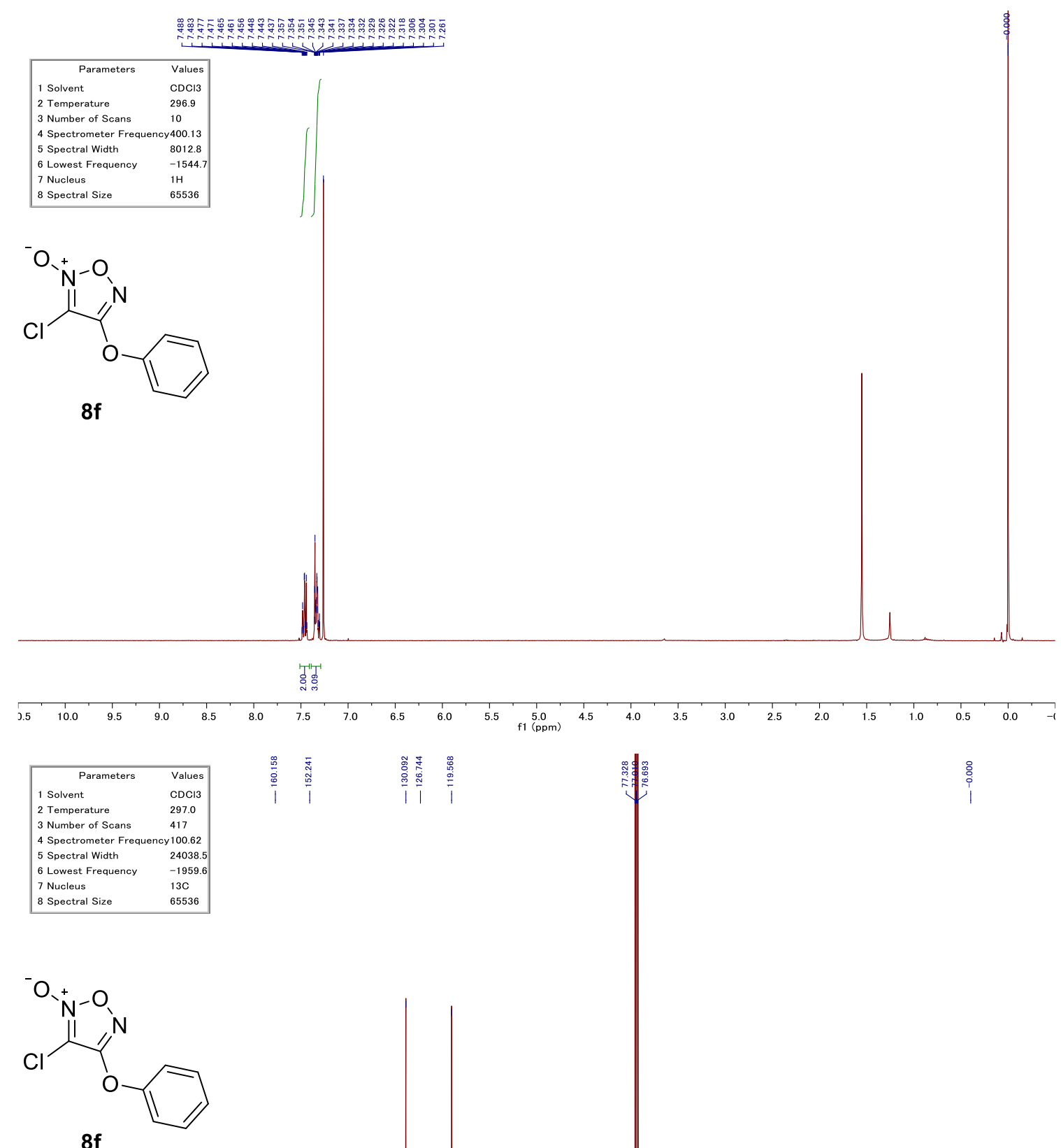

$8 f$

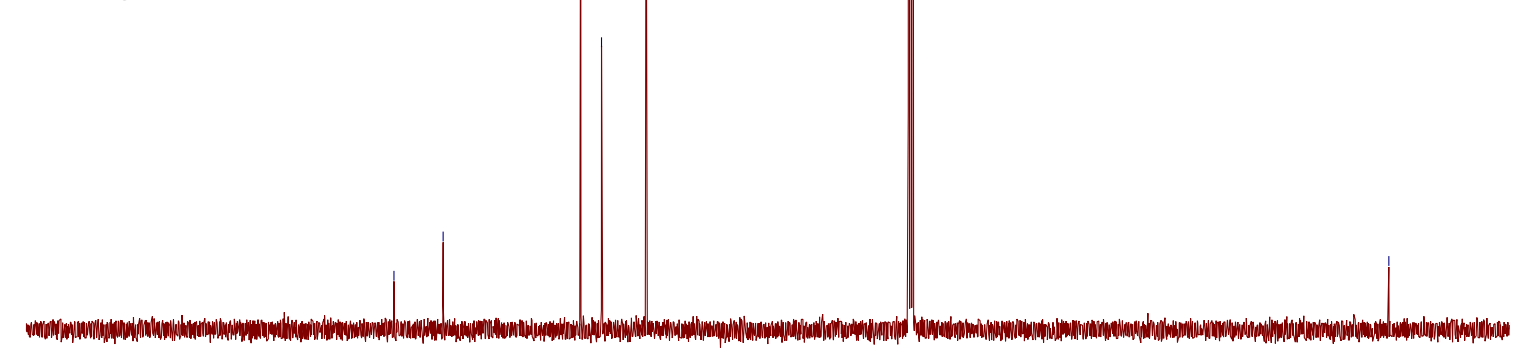




\begin{tabular}{|ll|}
\hline \multicolumn{1}{|c|}{ Parameters } & \multicolumn{1}{c|}{ Values } \\
1 Solvent & CDCI3 \\
2 Temperature & 290.1 \\
3 Number of Scans & 8 \\
4 Spectrometer Frequency400.1 \\
5 Spectral Width & 8012.8 \\
6 Lowest Frequency & -1542. \\
7 Nucleus & $1 \mathrm{H}$ \\
8 Spectral Size & 65536 \\
\hline
\end{tabular}<smiles>COc1no[n+]([O-])c1Br</smiles>

$11 a$
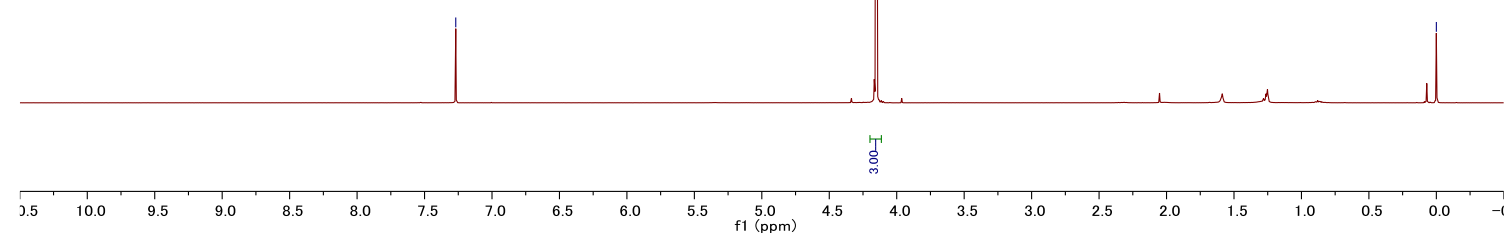

\begin{tabular}{|lc|}
\hline \multicolumn{1}{|c|}{ Parameters } & Values \\
1 Solvent & $\mathrm{CDCl} 3$ \\
2 Temperature & 290.9 \\
3 Number of Scans & 370 \\
4 Spectrometer Frequency 100.62 \\
5 Spectral Width & 24038.5 \\
6 Lowest Frequency & -1961.5 \\
7 Nucleus & $13 \mathrm{C}$ \\
8 Spectral Size & 65536 \\
\hline
\end{tabular}<smiles>COc1no[n+]([O-])c1Br</smiles>

$11 a$

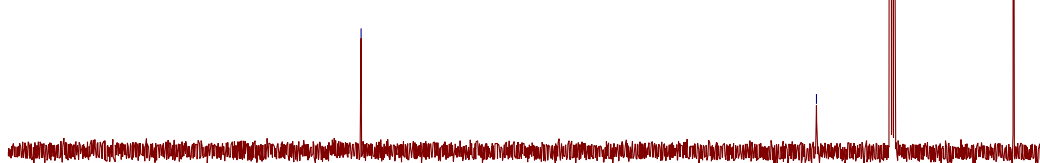



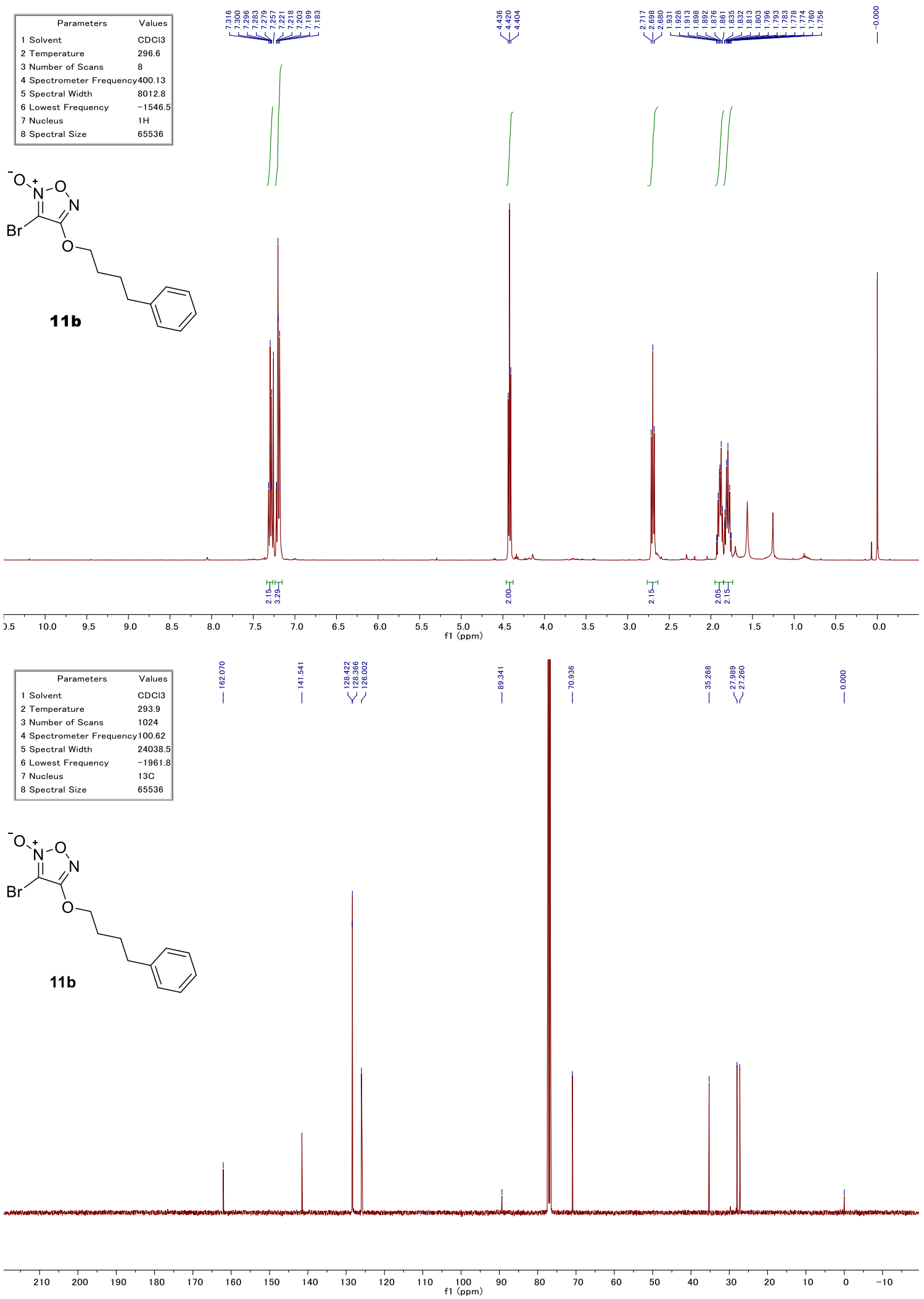

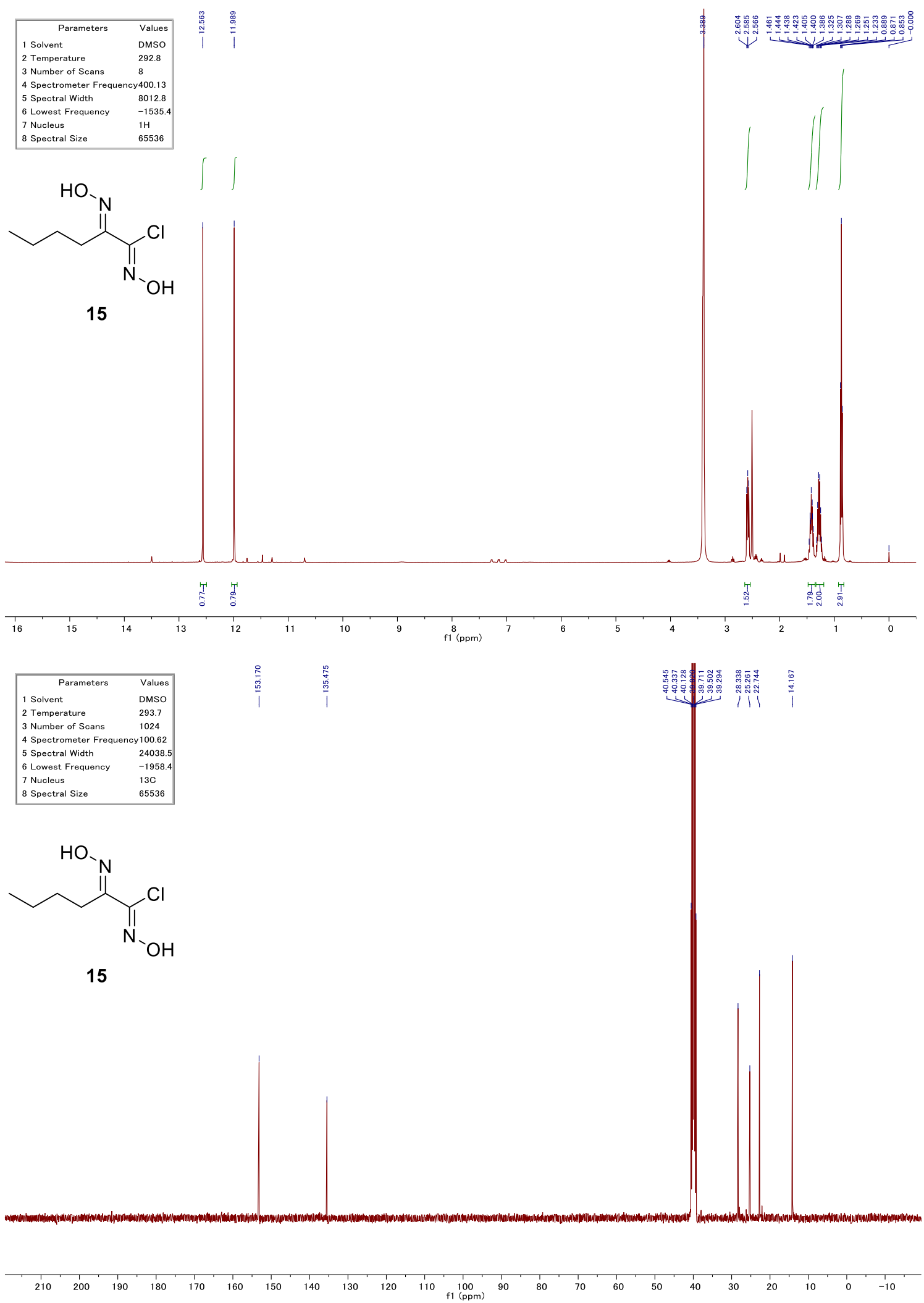

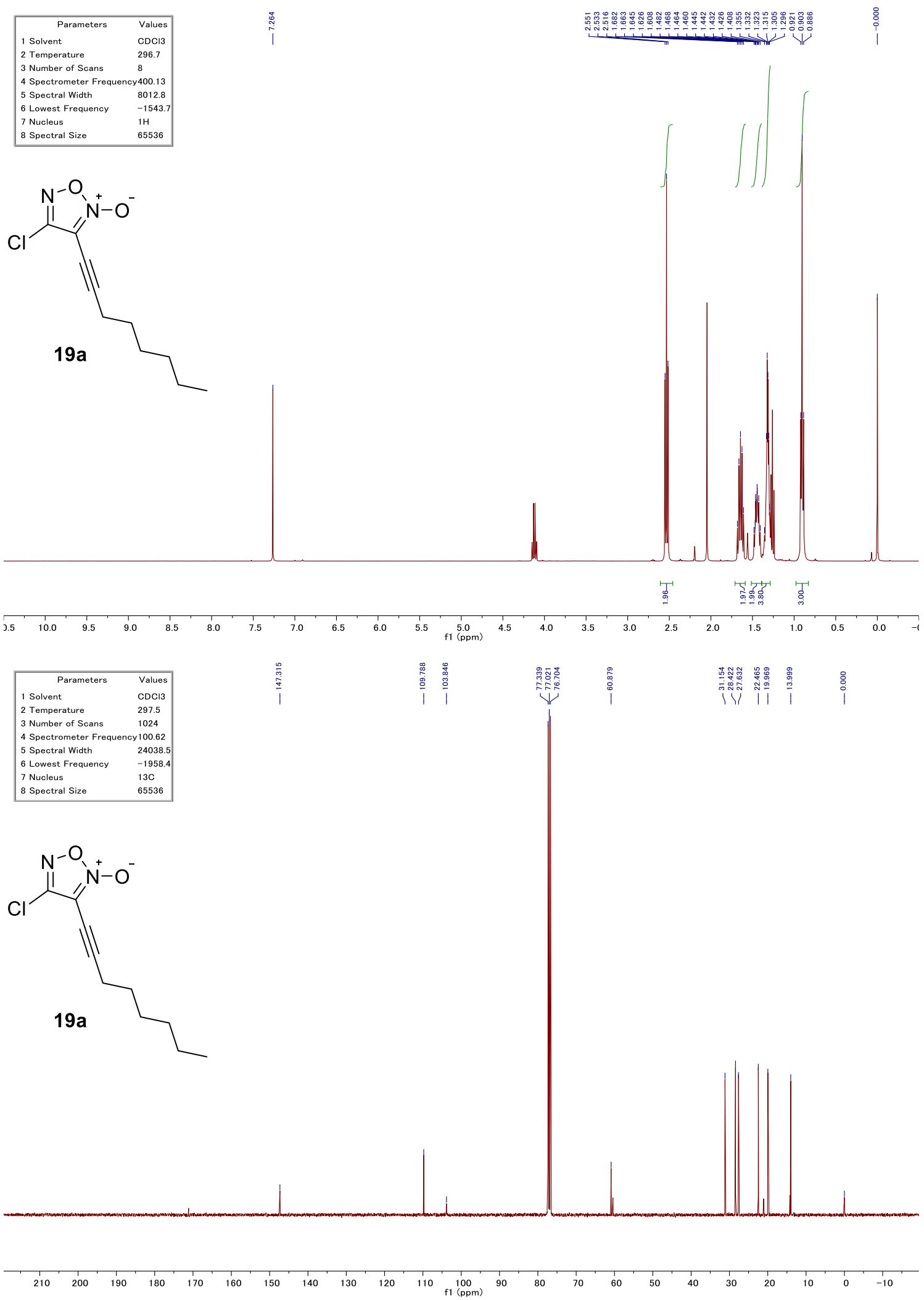


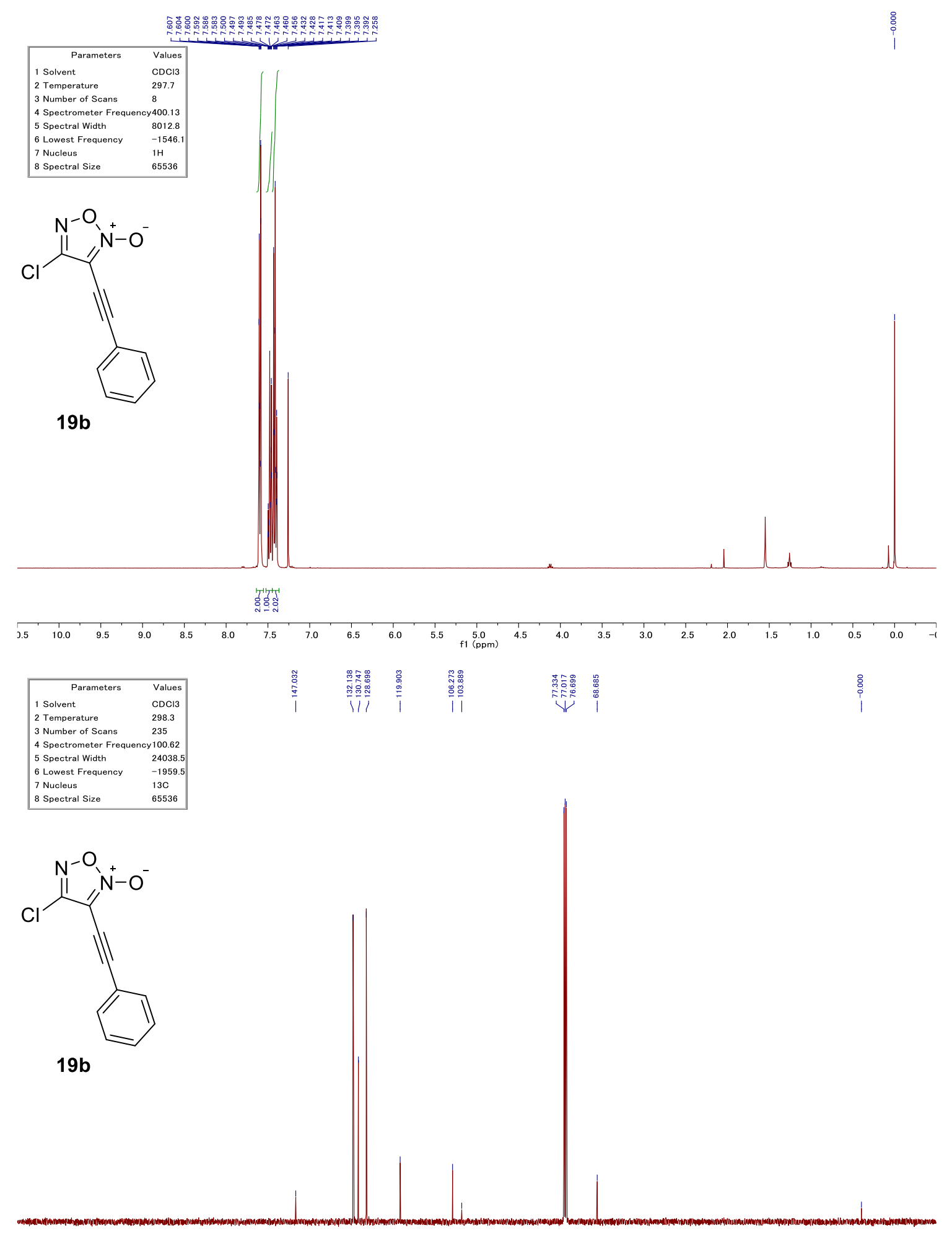

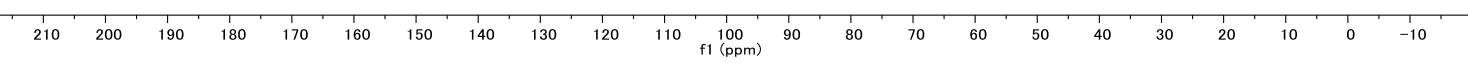



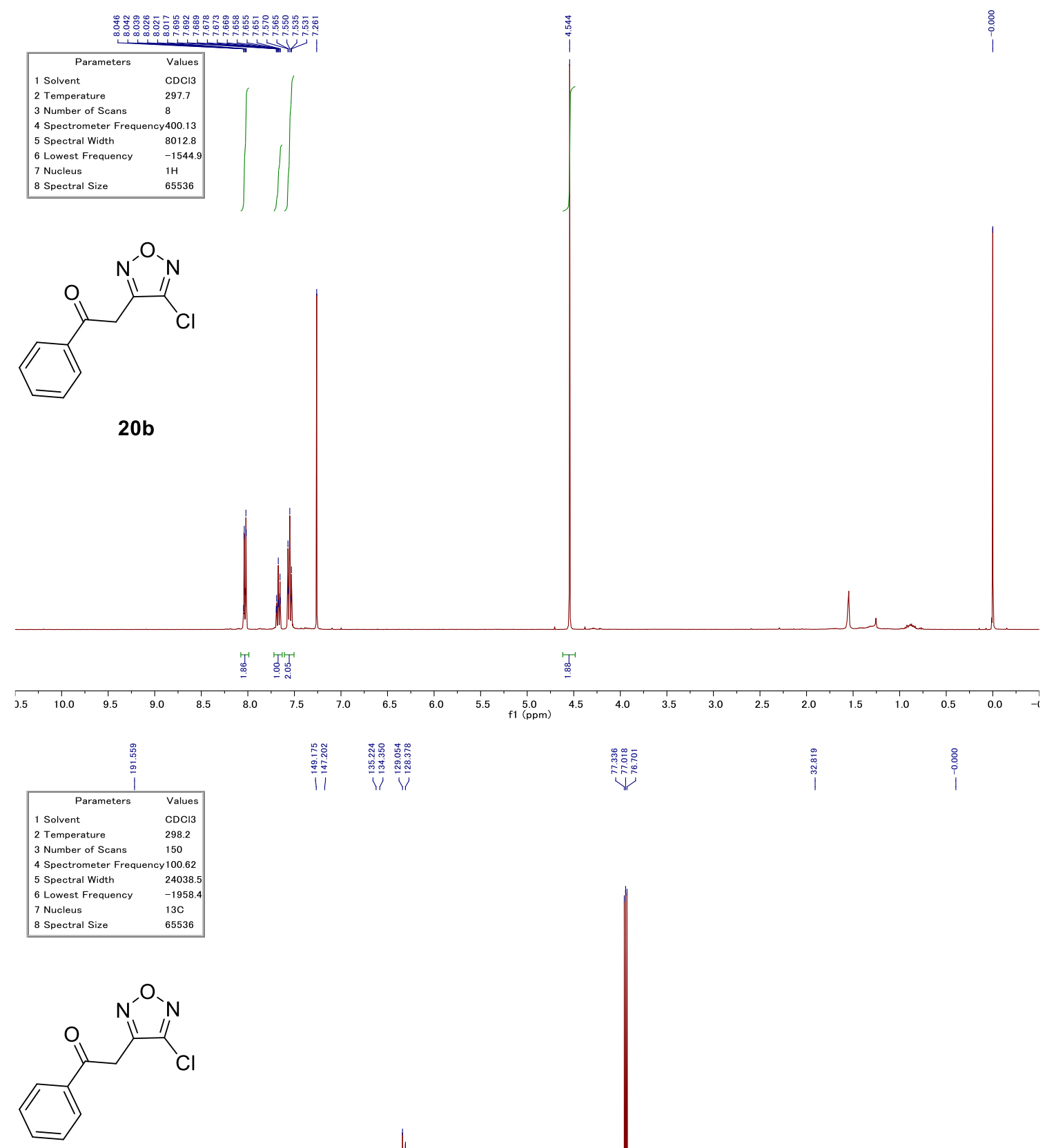

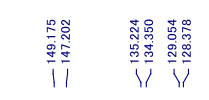

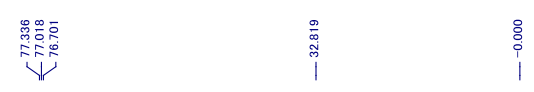

$20 b$

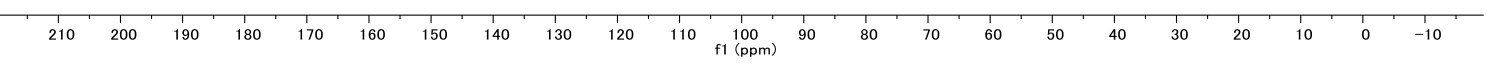




\begin{tabular}{|lc|}
\hline \multicolumn{1}{|c|}{ Parameters } & Values \\
1 Solvent & $\mathrm{CDCl}$ \\
2 Temperature & 297.6 \\
3 Number of Scans & 8 \\
4 Spectrometer Frequency 400.13 \\
5 Spectral Width & 8012.8 \\
6 Lowest Frequency & -1543.6 \\
7 Nucleus & $1 \mathrm{H}$ \\
8 Spectral Size & 65536 \\
\hline
\end{tabular}

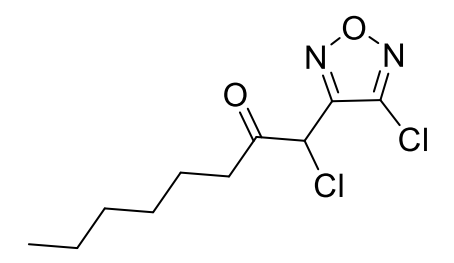

$21 \mathrm{a}$
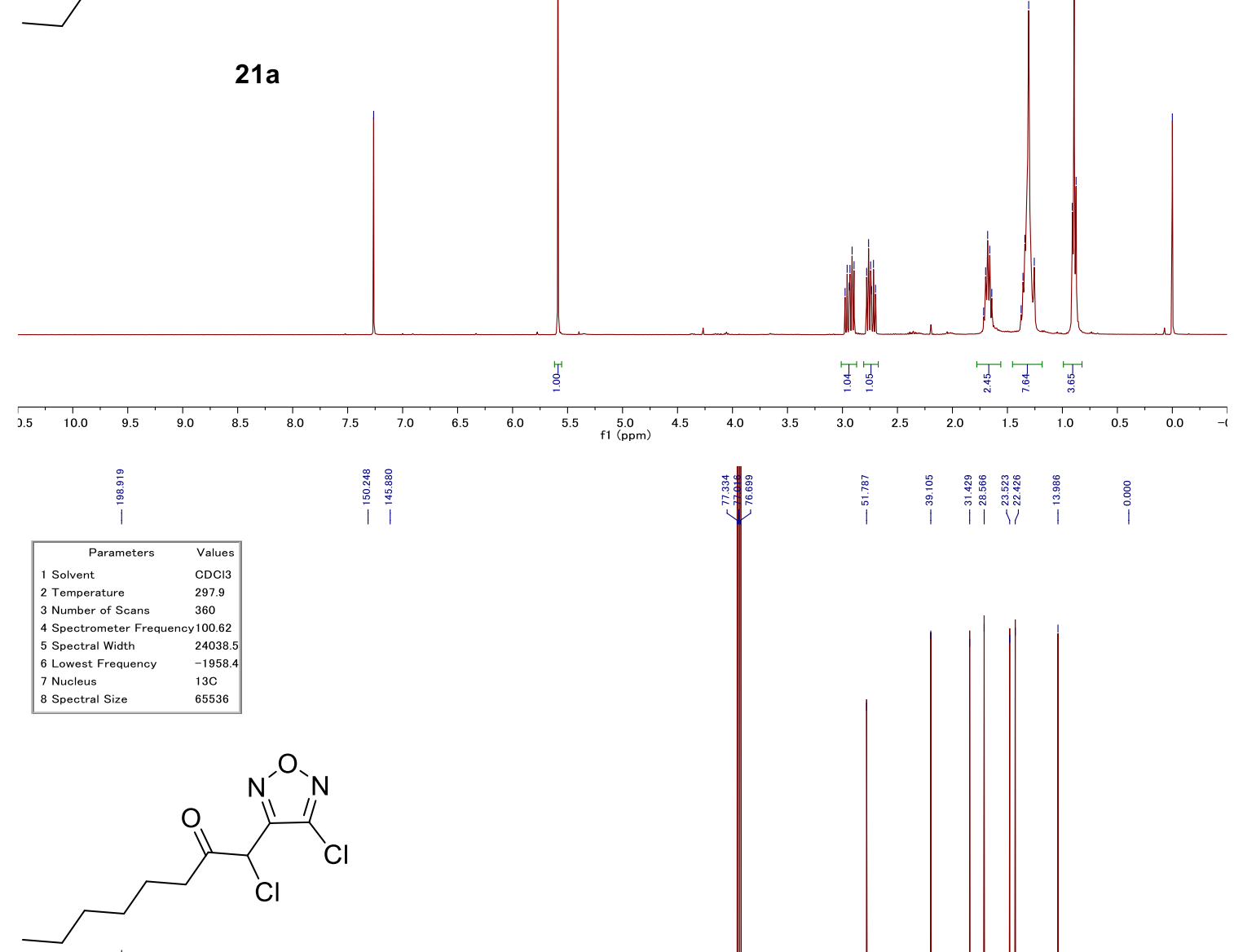

II

$21 \mathrm{a}$

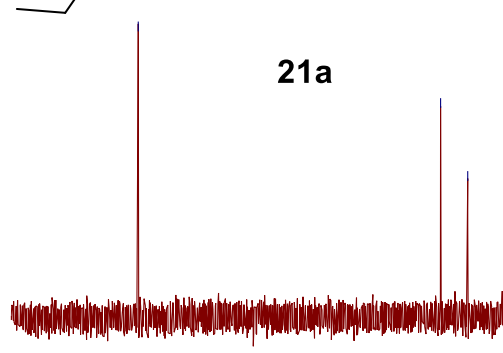

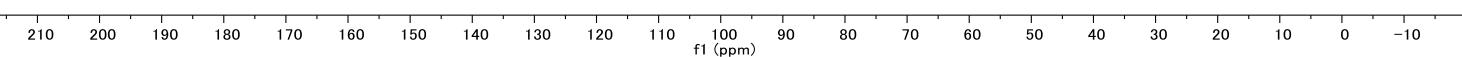



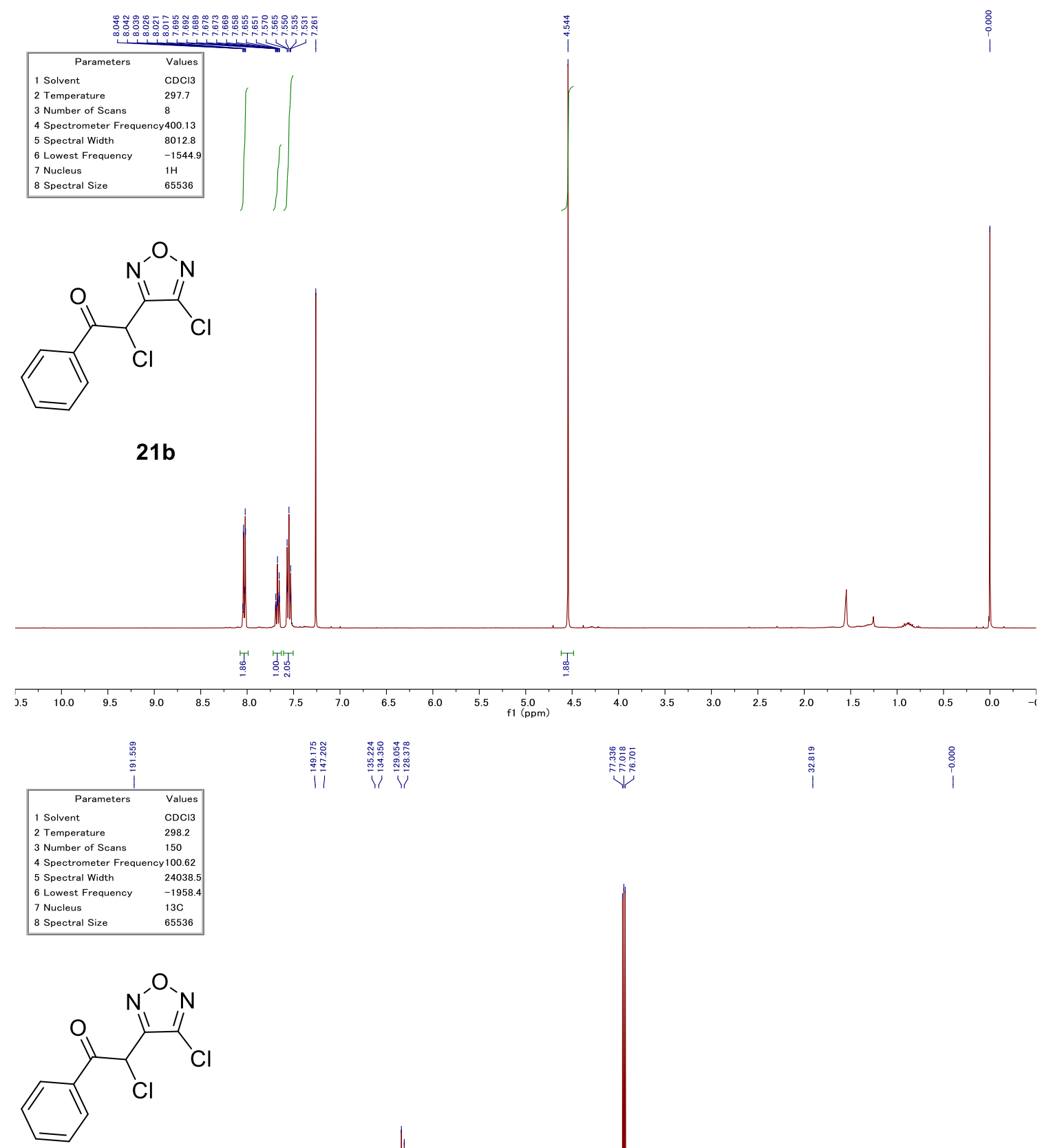

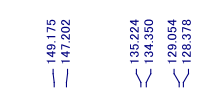

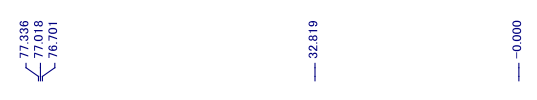

21b

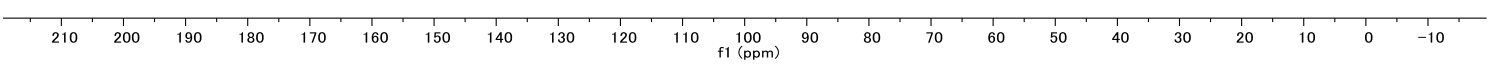



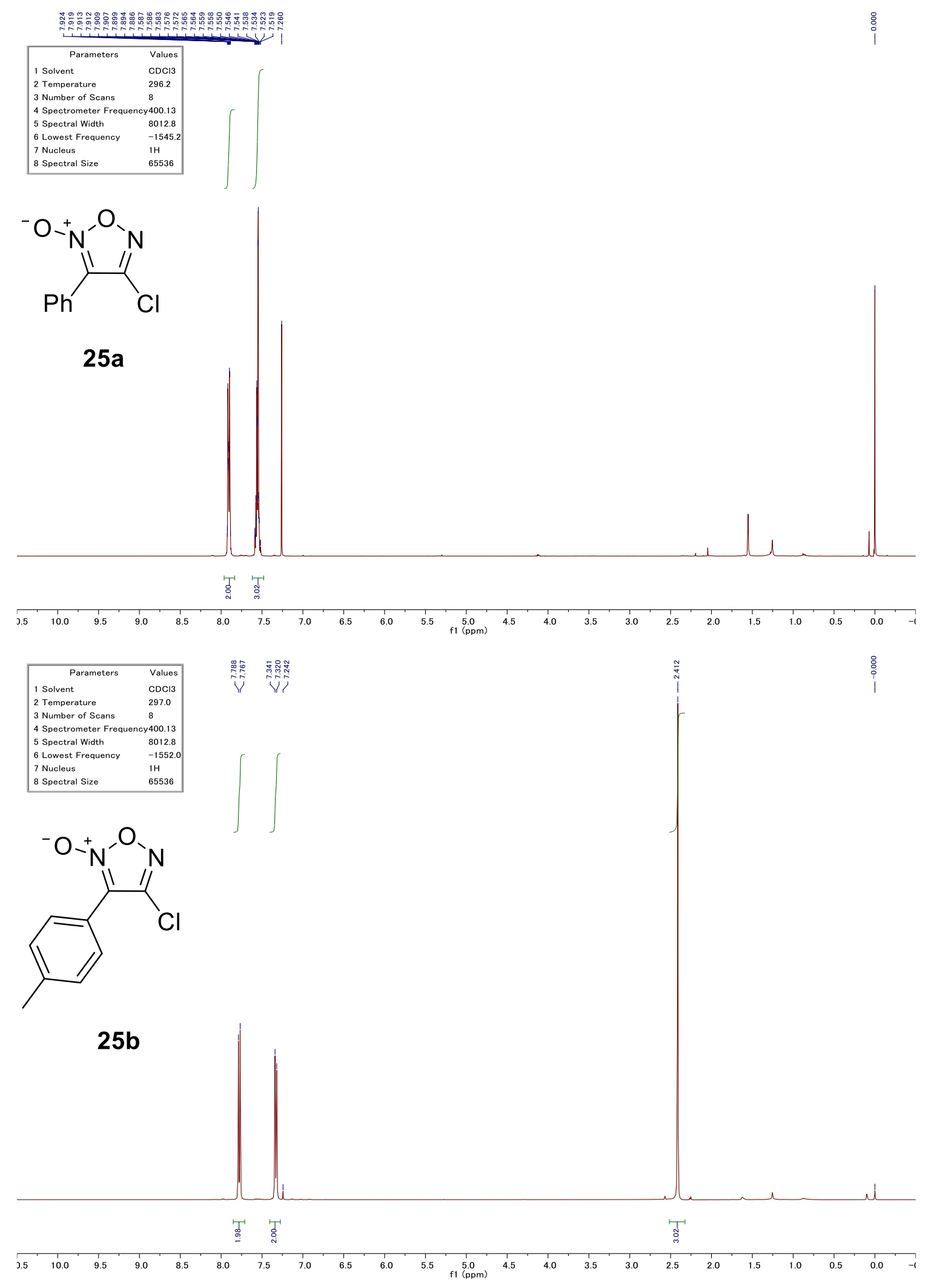

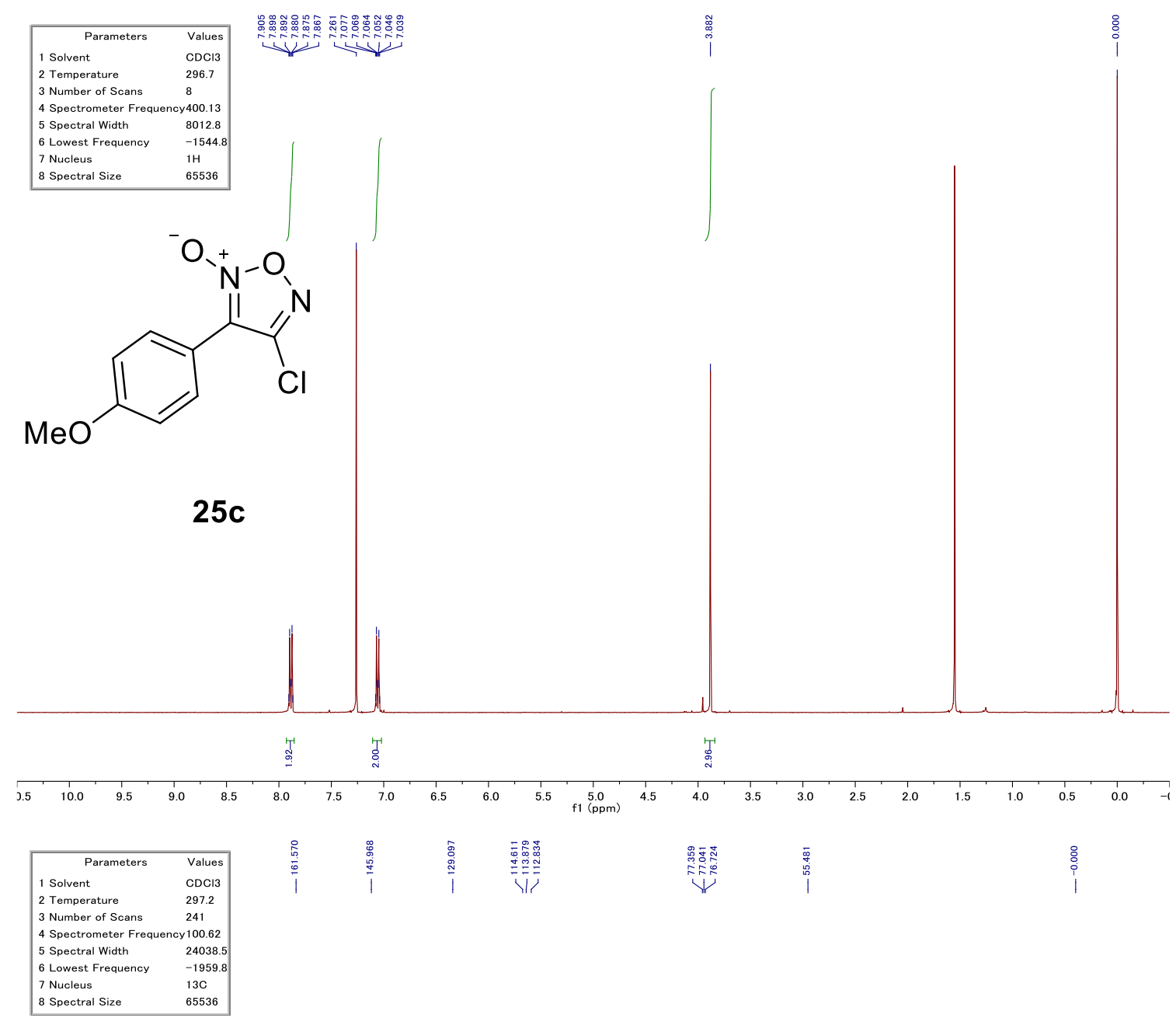

(c)

25c

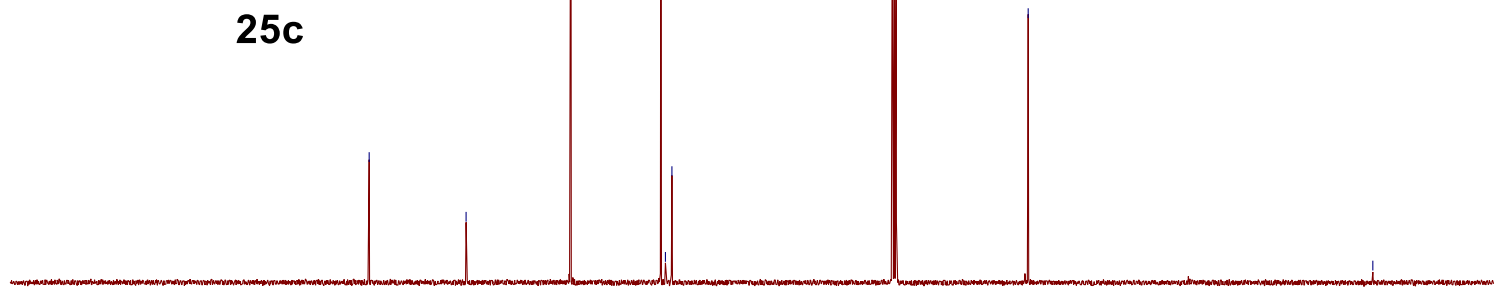

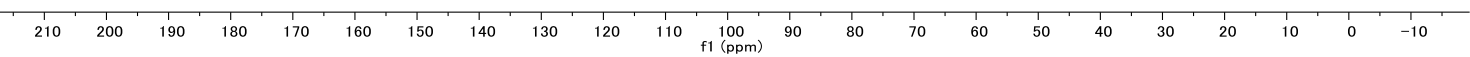


\begin{tabular}{|lc|}
\hline \multicolumn{1}{|c|}{ Parameters } & Values \\
1 Solvent & $\mathrm{CDC13}$ \\
2 Temperature & 297.6 \\
3 Number of Scans & 8 \\
4 Spectrometer Frequency 400.13 \\
5 Spectral Width & 8012.8 \\
6 Lowest Frequency & -1544 \\
7 Nucleus & $1 \mathrm{H}$ \\
8 Spectral Size & 65536 \\
\hline
\end{tabular}<smiles>CCCCCc1c(Br)no[n+]1[O-]</smiles>

$27 a$

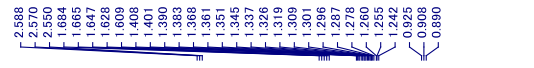

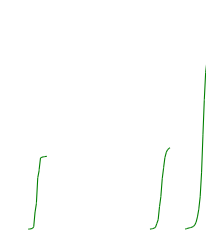

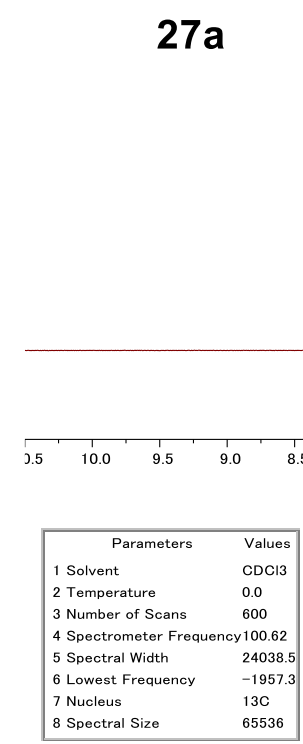<smiles>CCCCCc1c(Br)no[n+]1[O-]</smiles>

$27 a$

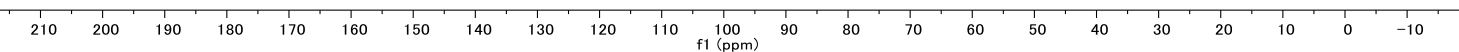



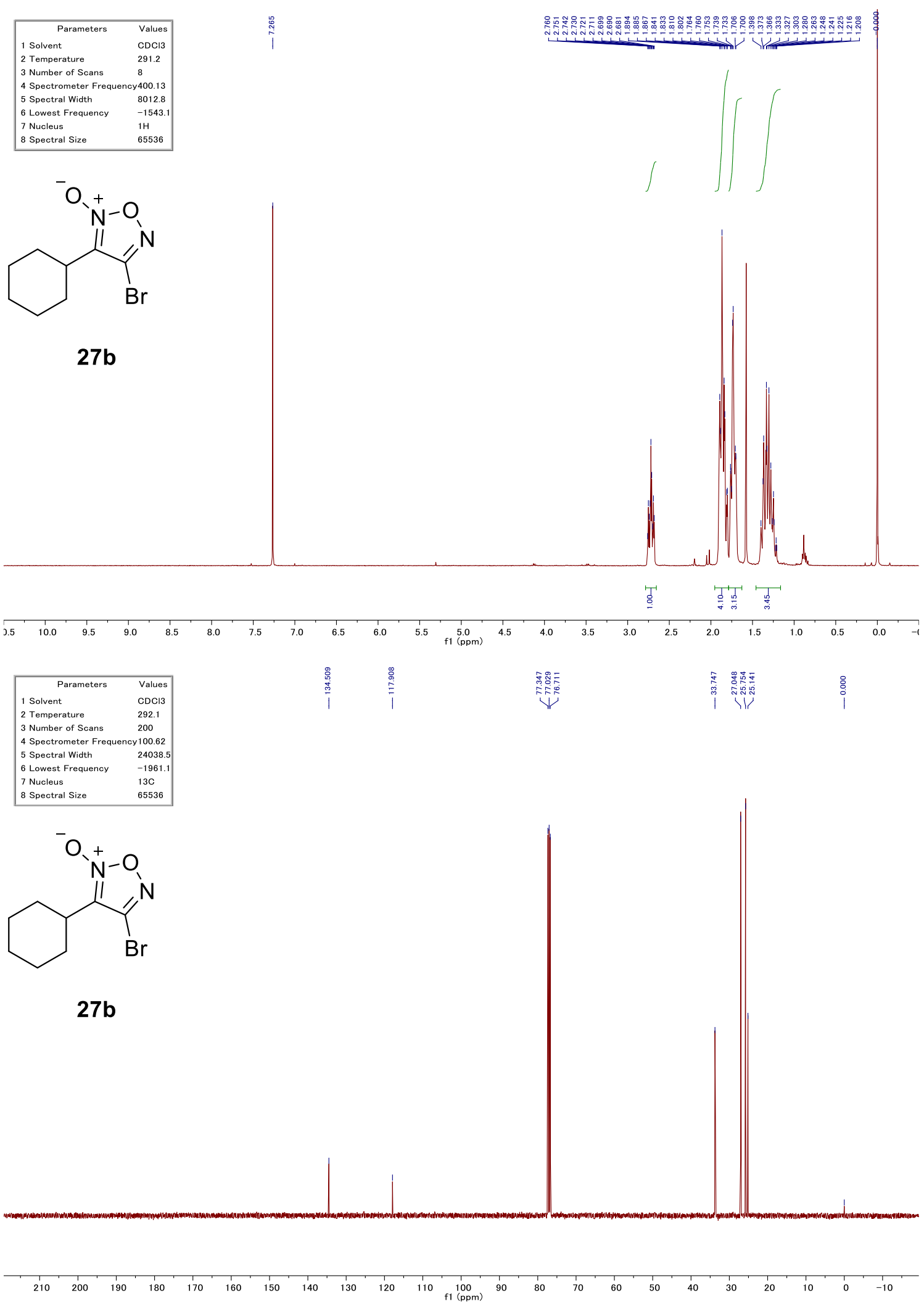

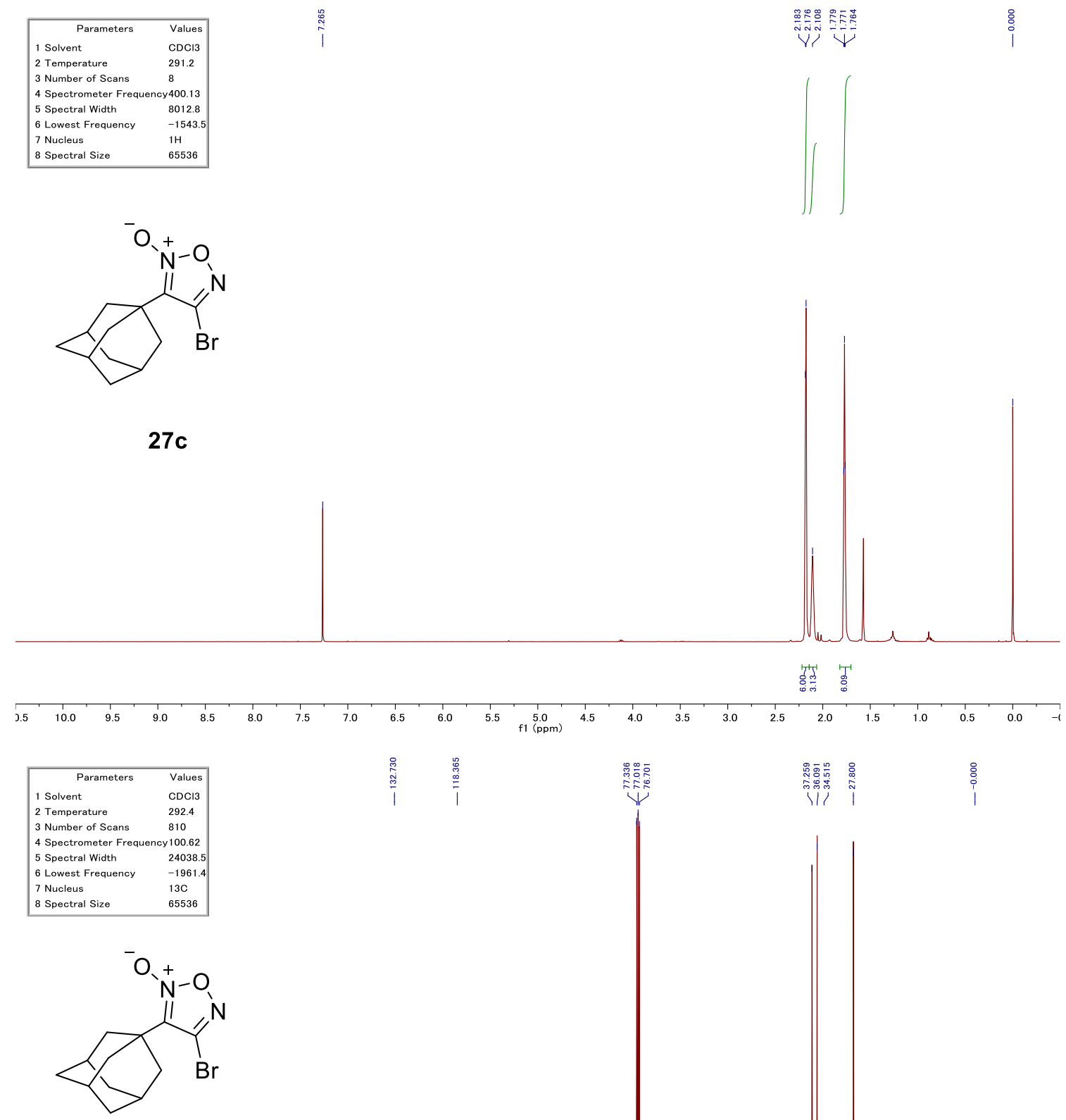

$27 c$

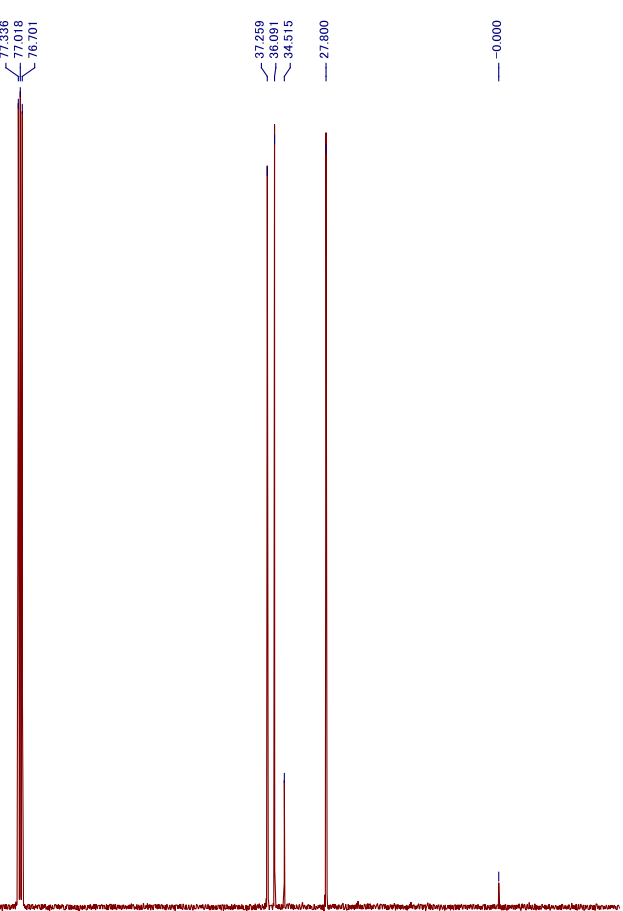

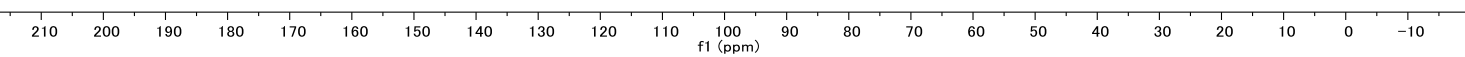




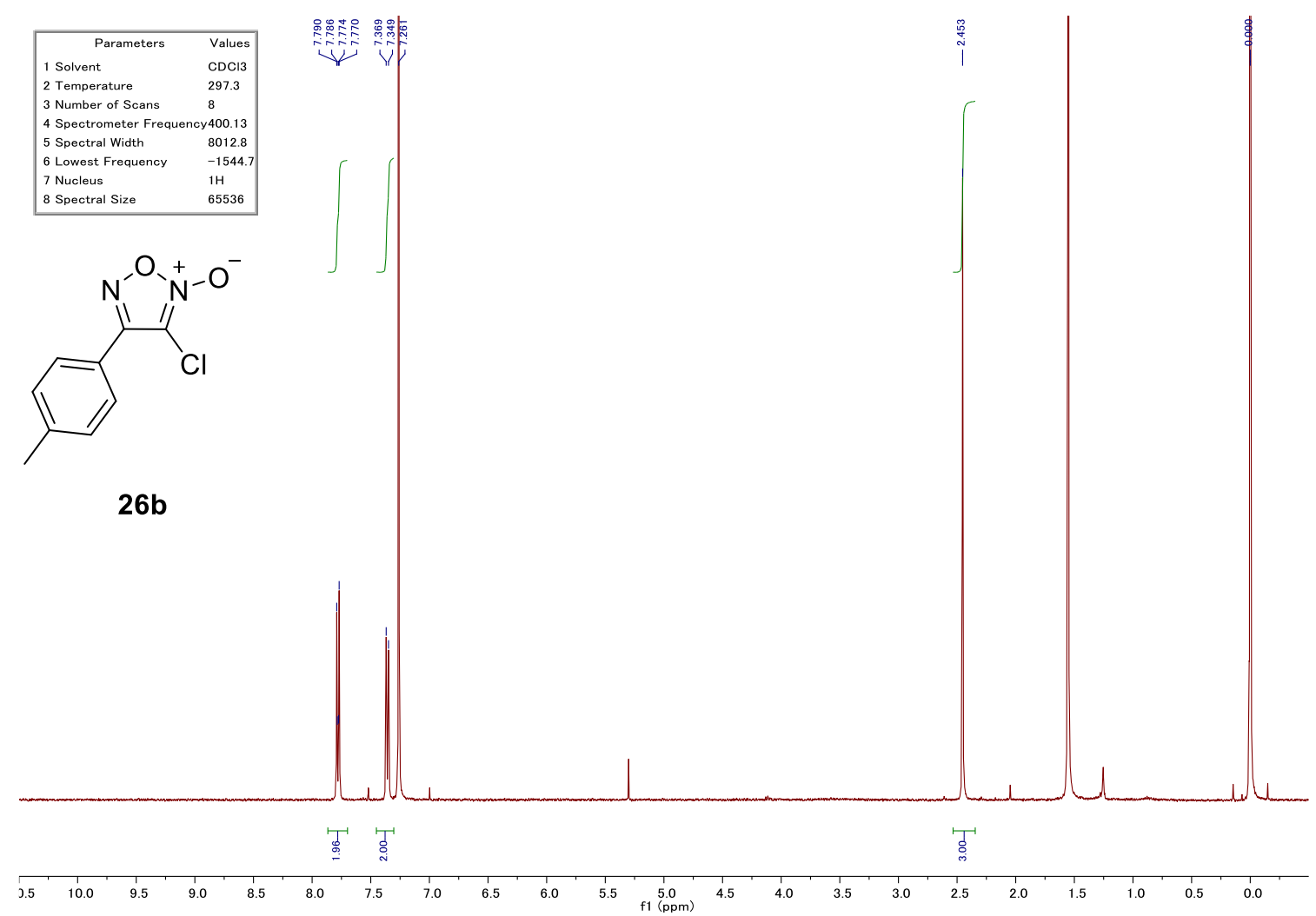



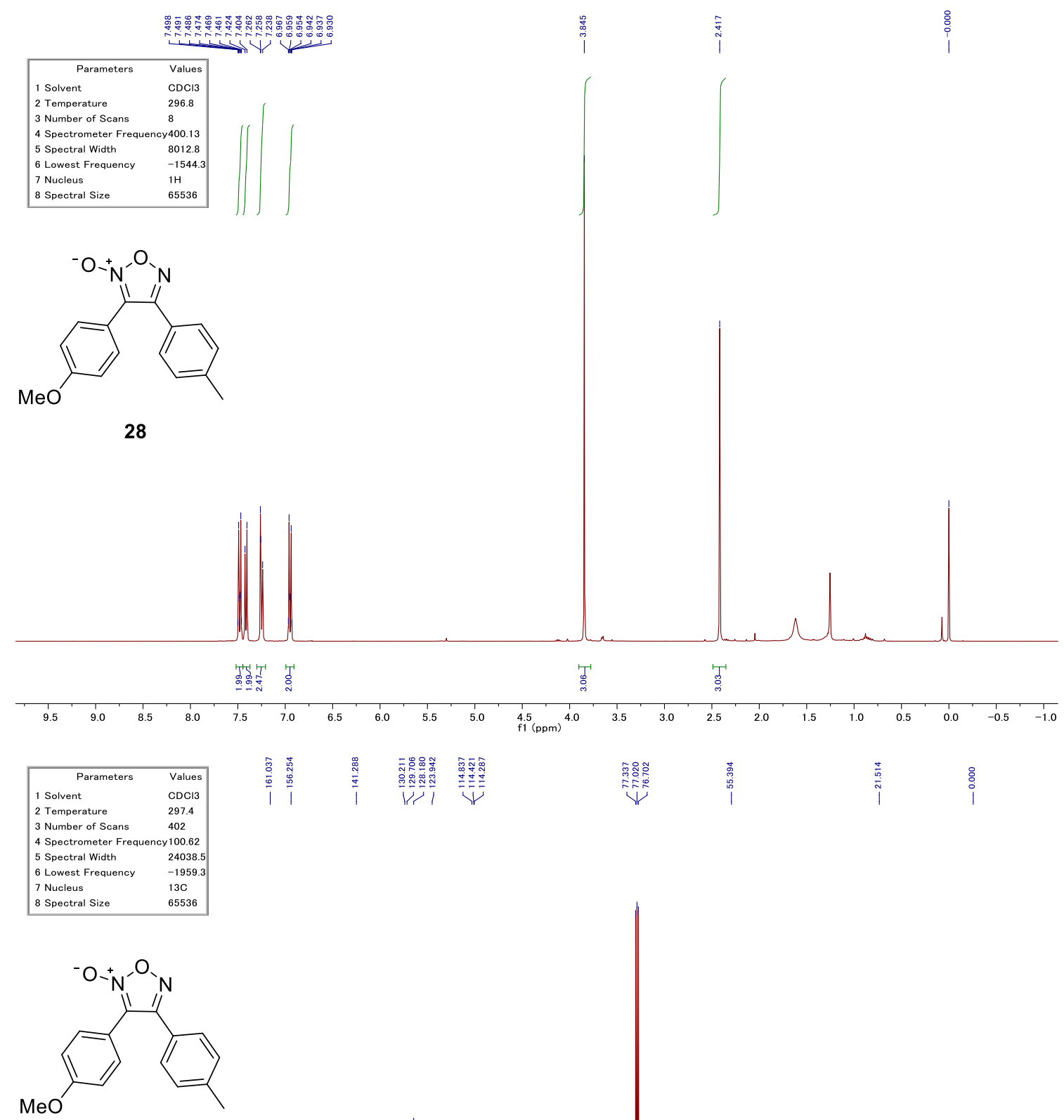

28 


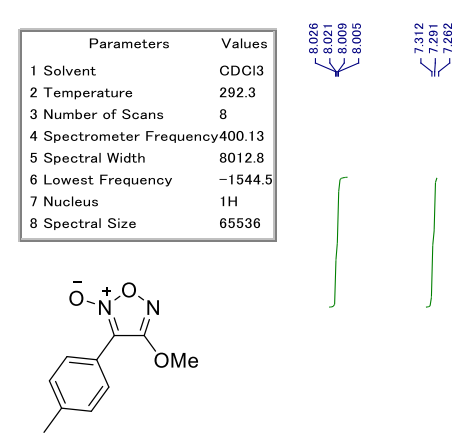

29

Isolated authentic sample

(synthesized in the

previous work)
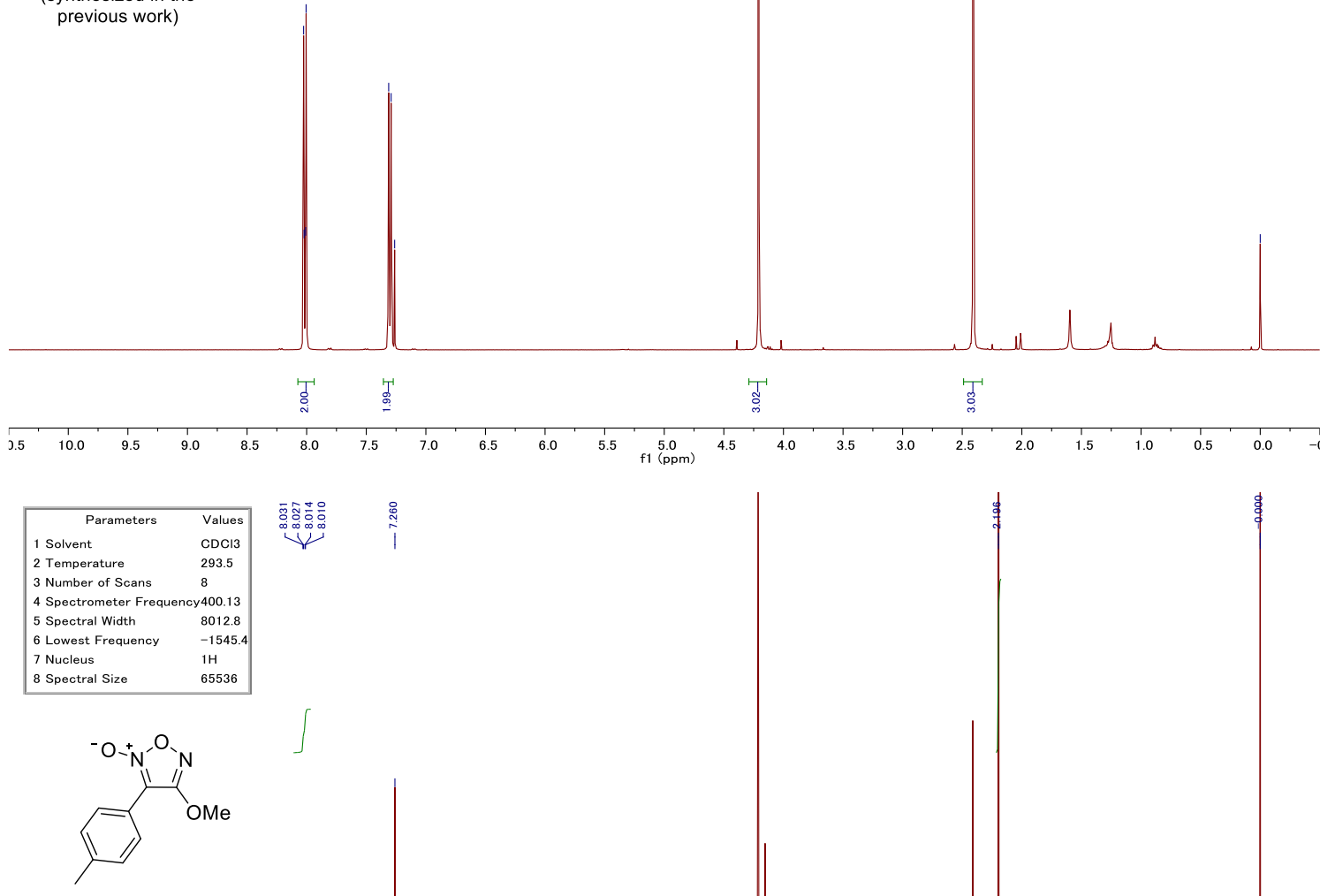

29

Crude material with durene (IS)

in the reaction from $25 b$ to 29

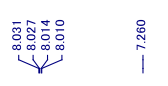

12.8

536 


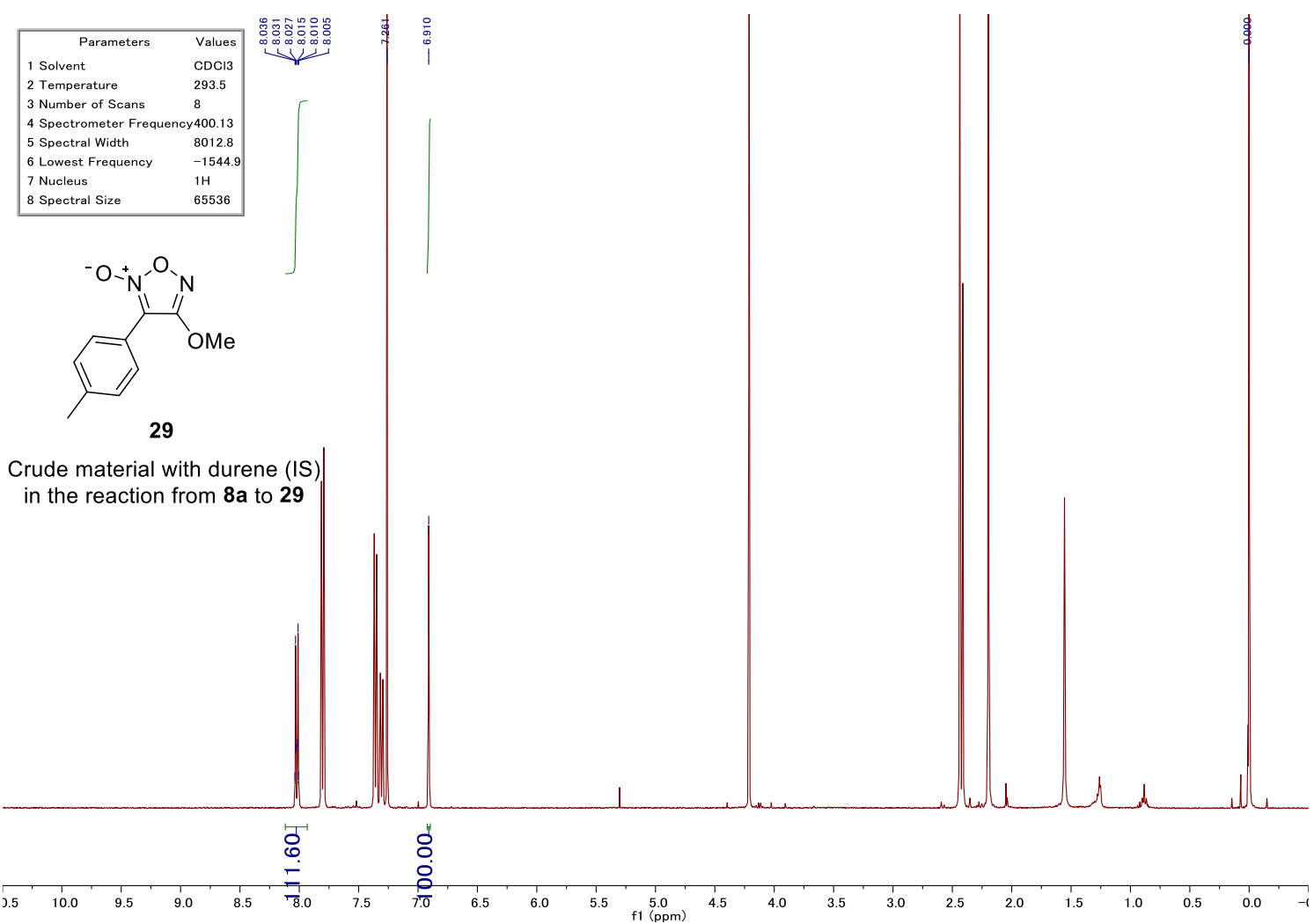



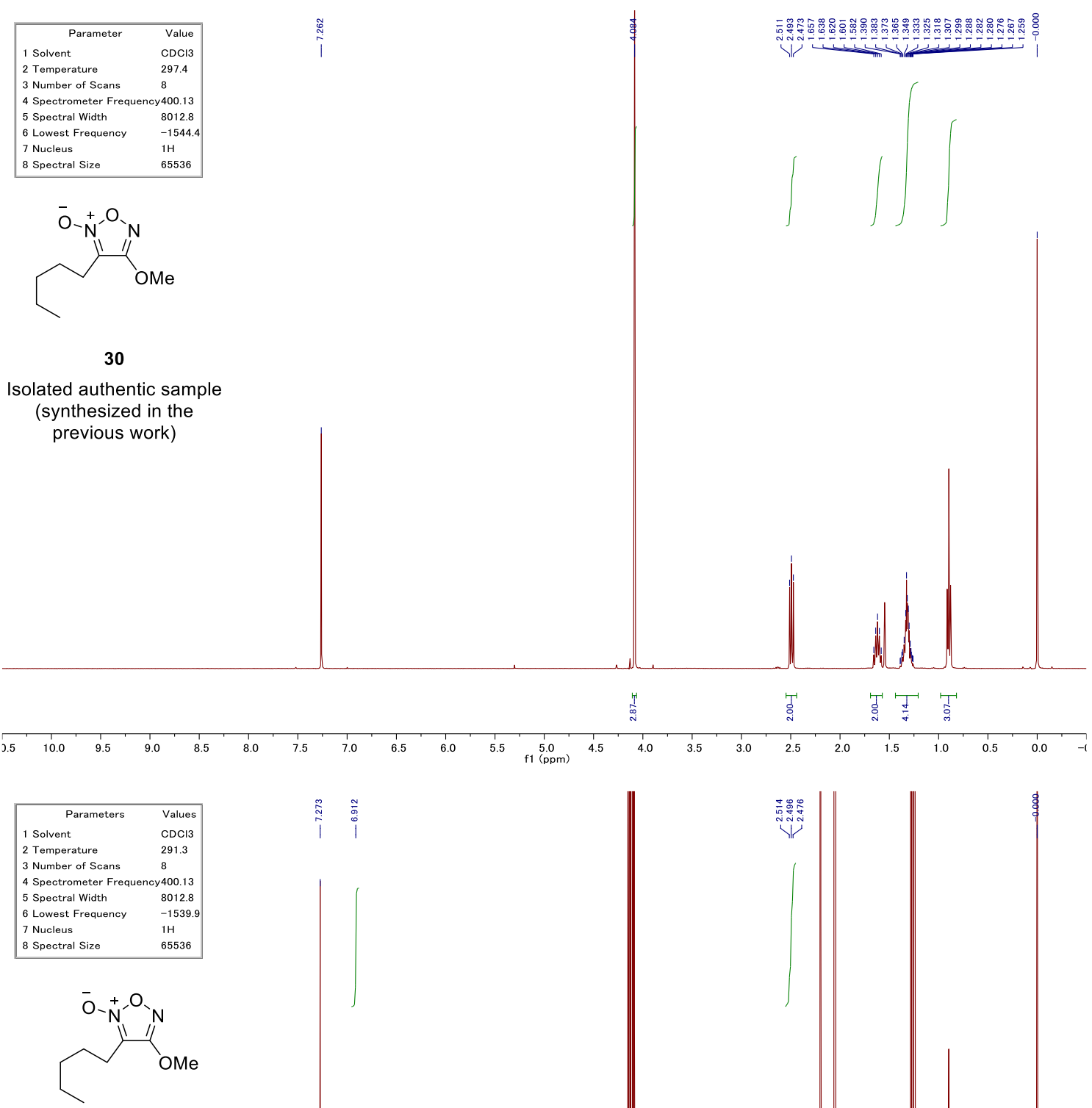

30

Crude material with durene (IS)

in the reaction from $11 \mathrm{a}$ to 30

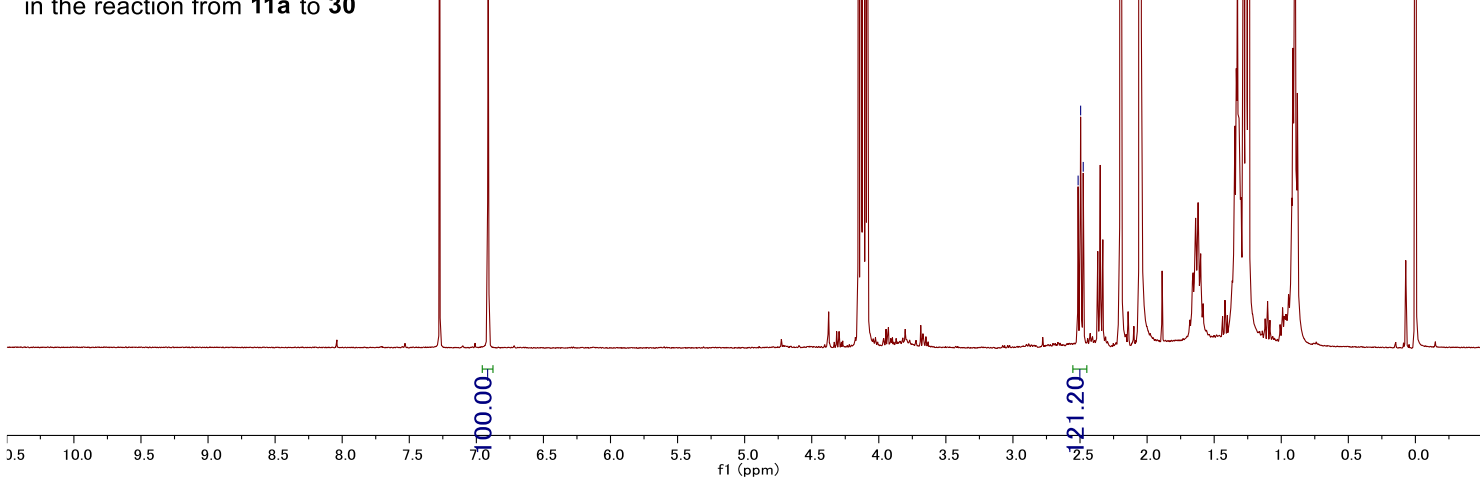




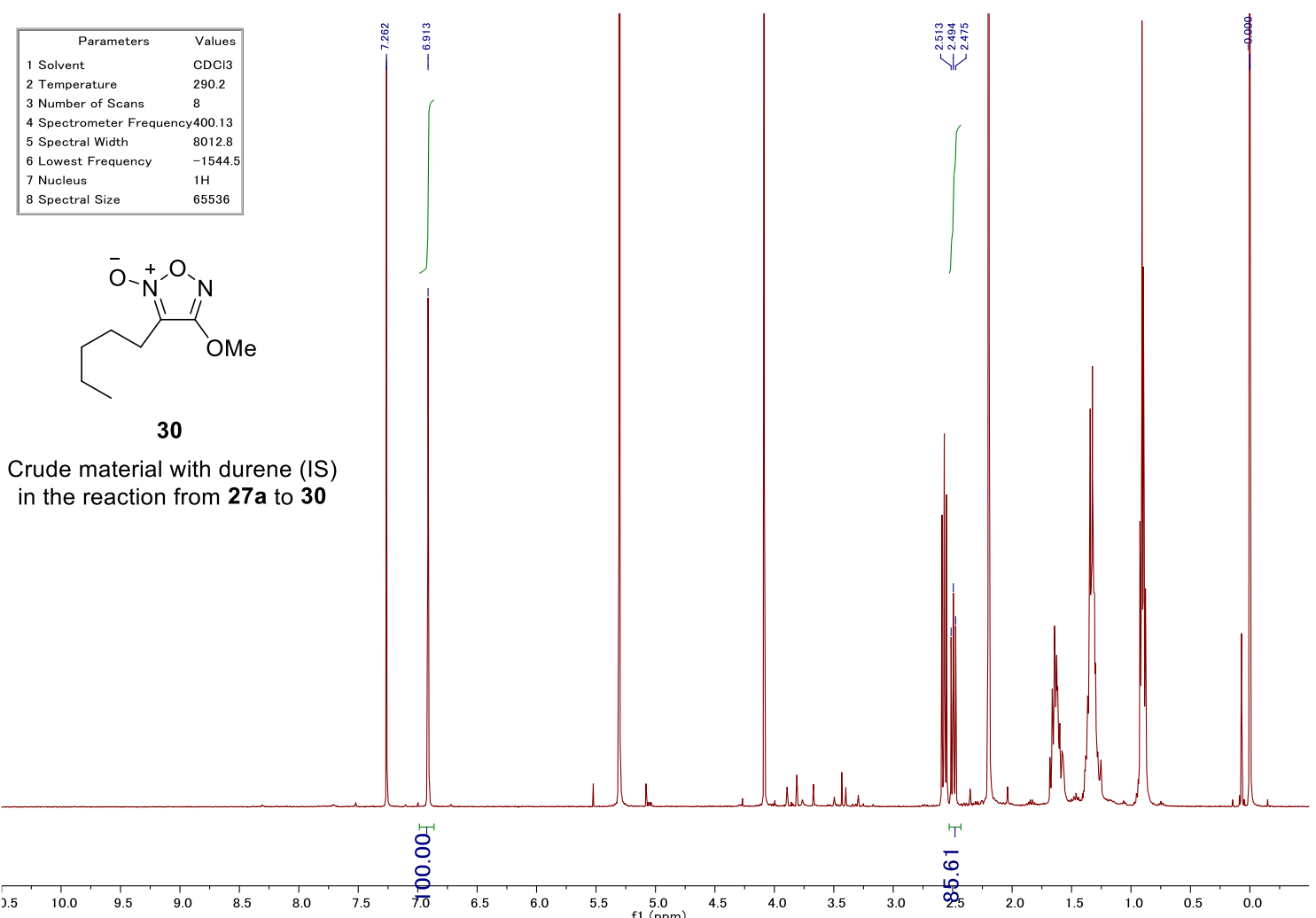


\begin{tabular}{|lc|}
\hline \multicolumn{1}{|c|}{ Parameters } & \multicolumn{1}{c|}{ Values } \\
1 Solvent & $\mathrm{CDCl3}$ \\
2 Temperature & 294.2 \\
3 Number of Scans & 8 \\
4 Spectrometer Frequency 400.13 \\
5 Spectral Width & 8012.8 \\
6 Lowest Frequency & -1543.9 \\
7 Nucleus & $1 \mathrm{H}$ \\
8 Spectral Size & 65536 \\
\hline
\end{tabular}

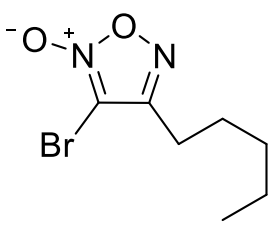

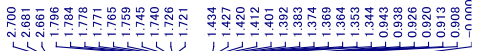

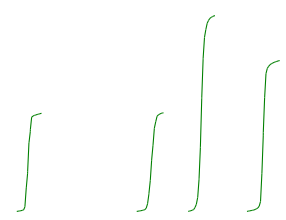

31

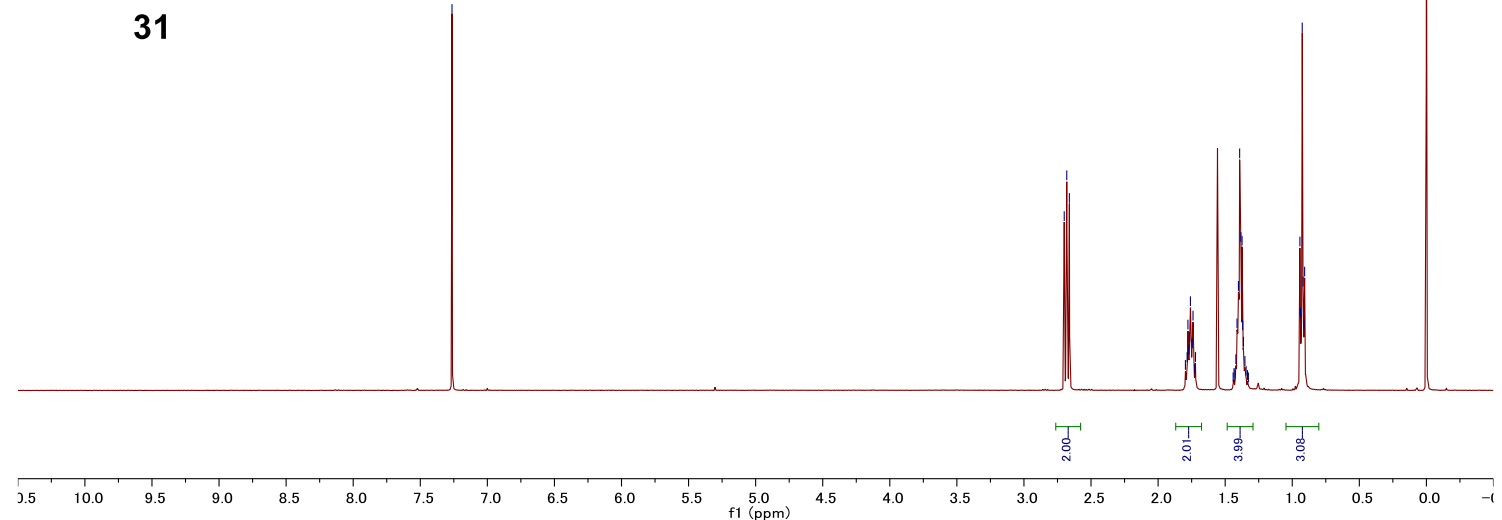

\begin{tabular}{|lc|}
\hline \multicolumn{1}{|c|}{ Parameters } & Values \\
1 Solvent & $\mathrm{CDCl3}$ \\
2 Temperature & 297.0 \\
3 Number of Scans & 1088 \\
4 Spectrometer Frequency 100.62 \\
5 Spectral Width & 24038.5 \\
6 Lowest Frequency & -1959.1 \\
7 Nucleus & $13 \mathrm{C}$ \\
8 Spectral Size & 65536 \\
\hline
\end{tabular}

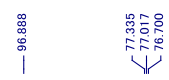

|

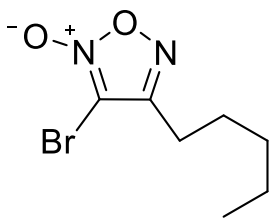

31

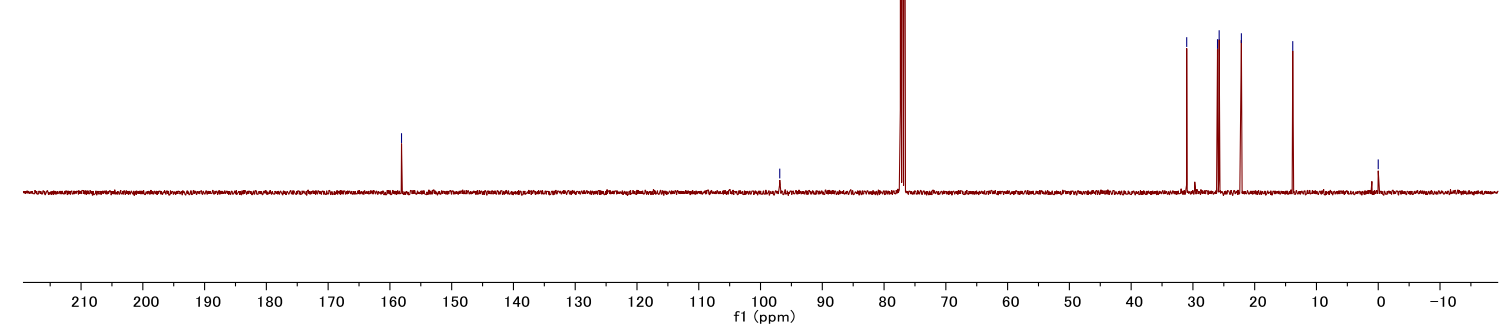




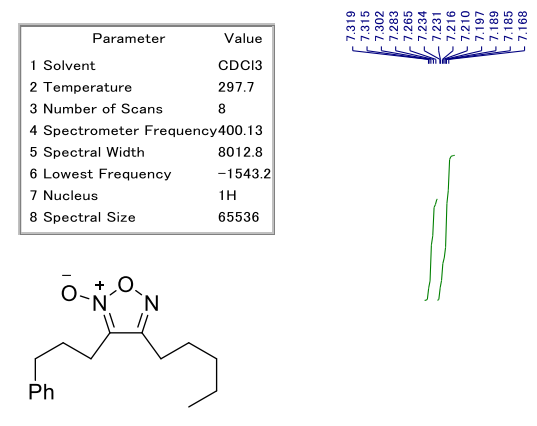

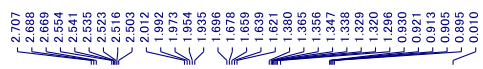

$32 a$

Isolated authentic sample (synthesized in the previous work)
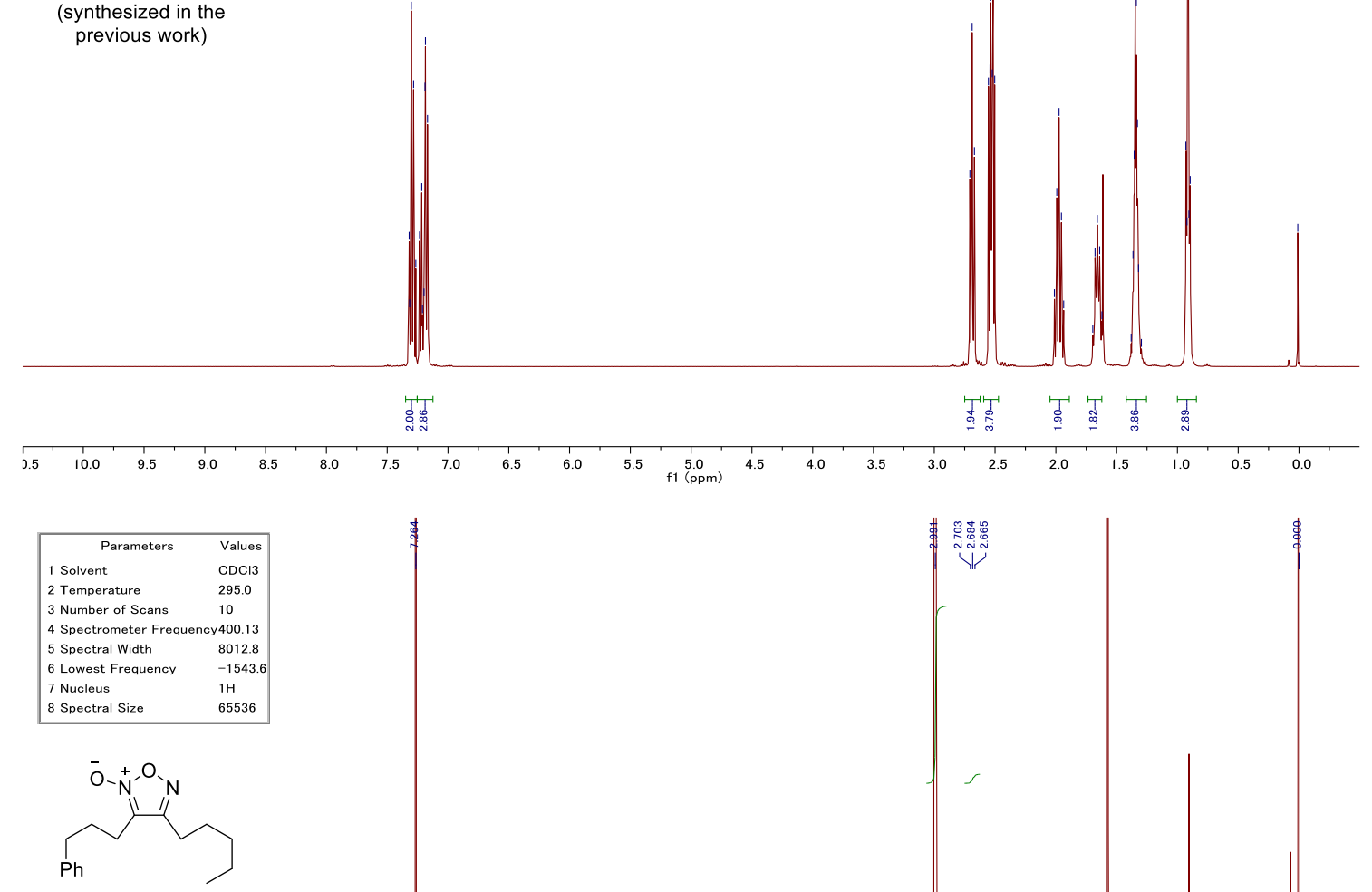

$32 \mathrm{a}$

Crude material with

dimethylsulfone (IS)

in the reaction from 31 to 32
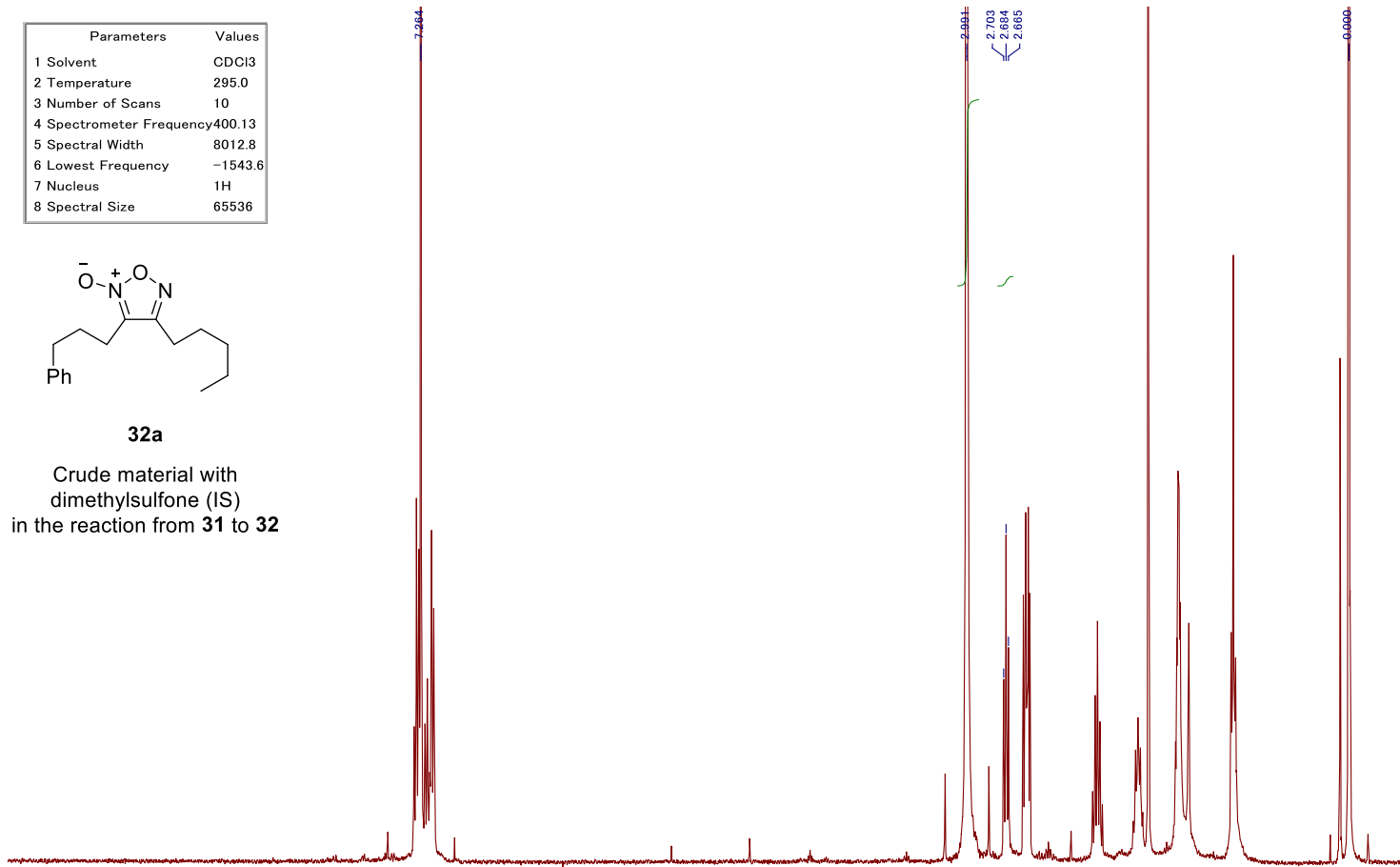

's' 'T
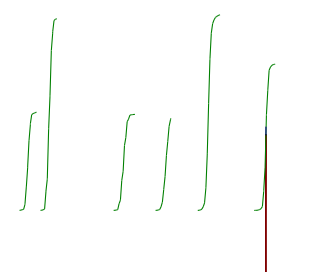

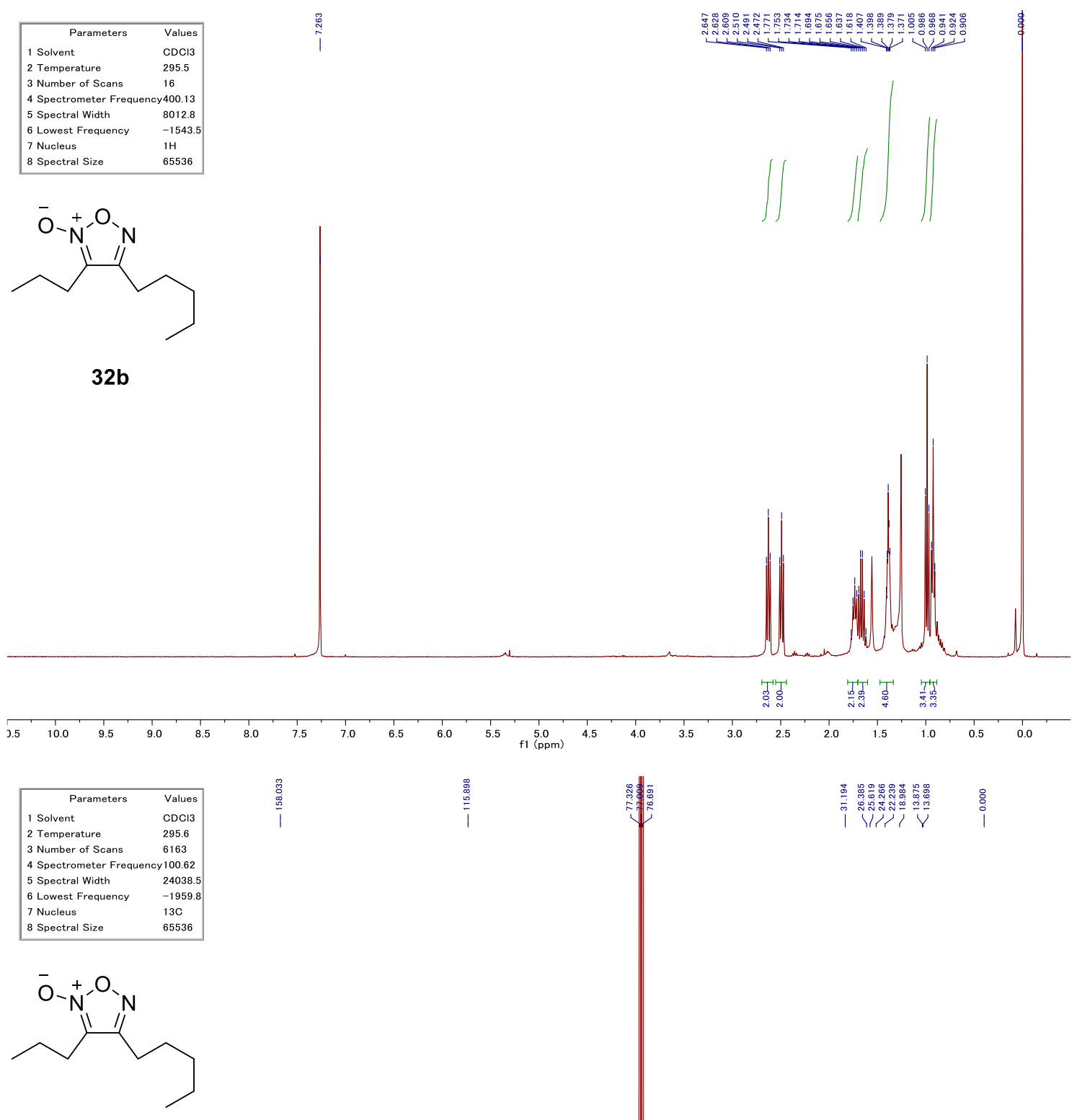

32b

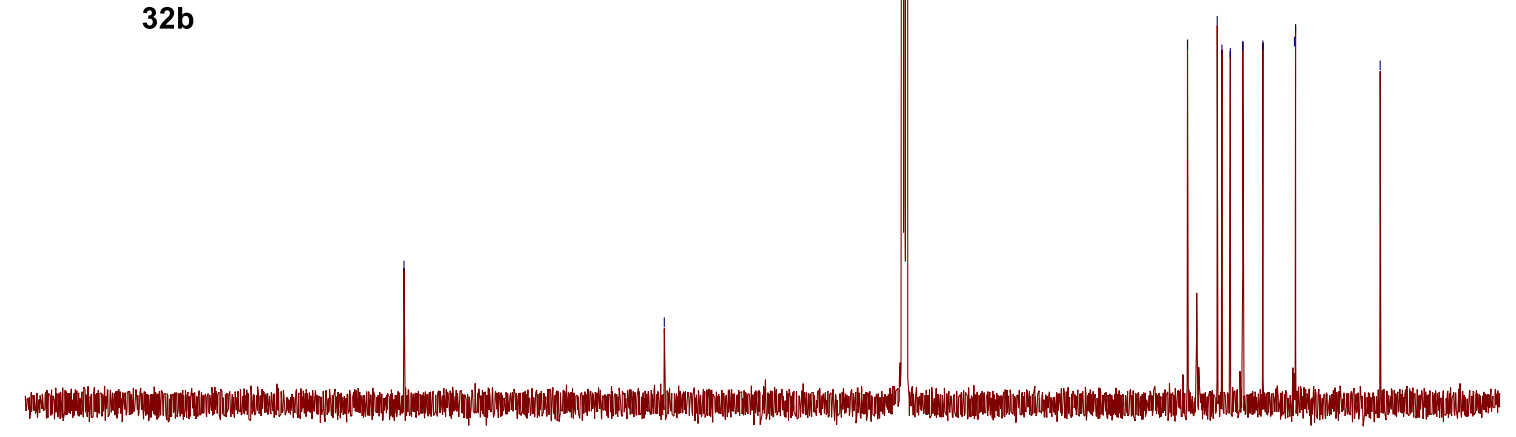



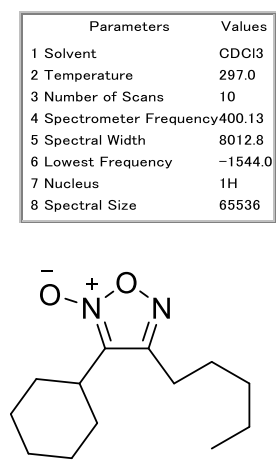

32c

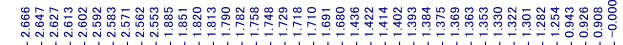

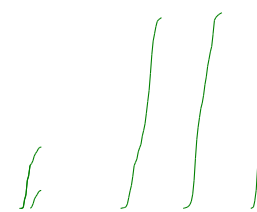

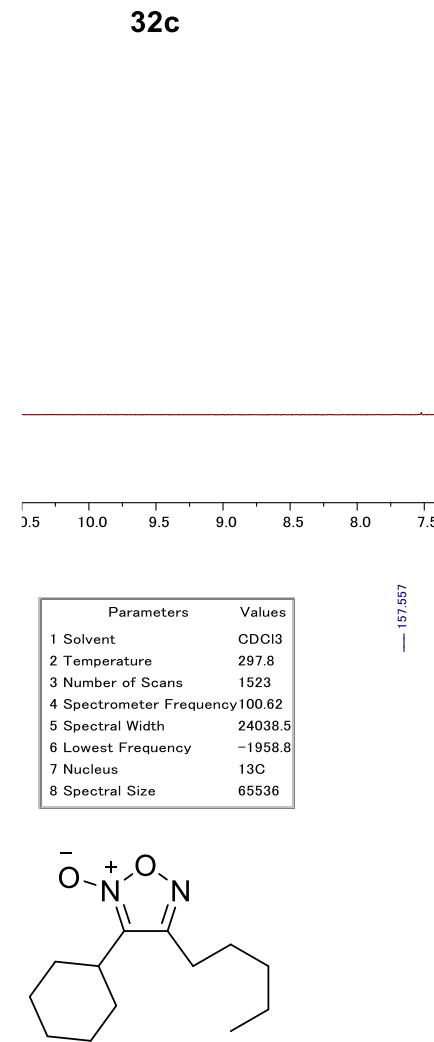

$32 \mathrm{c}$

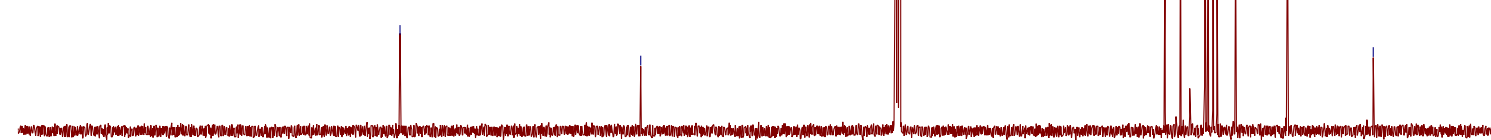

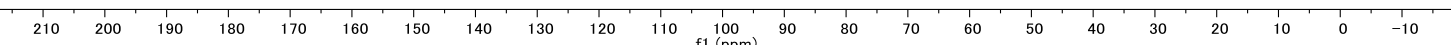



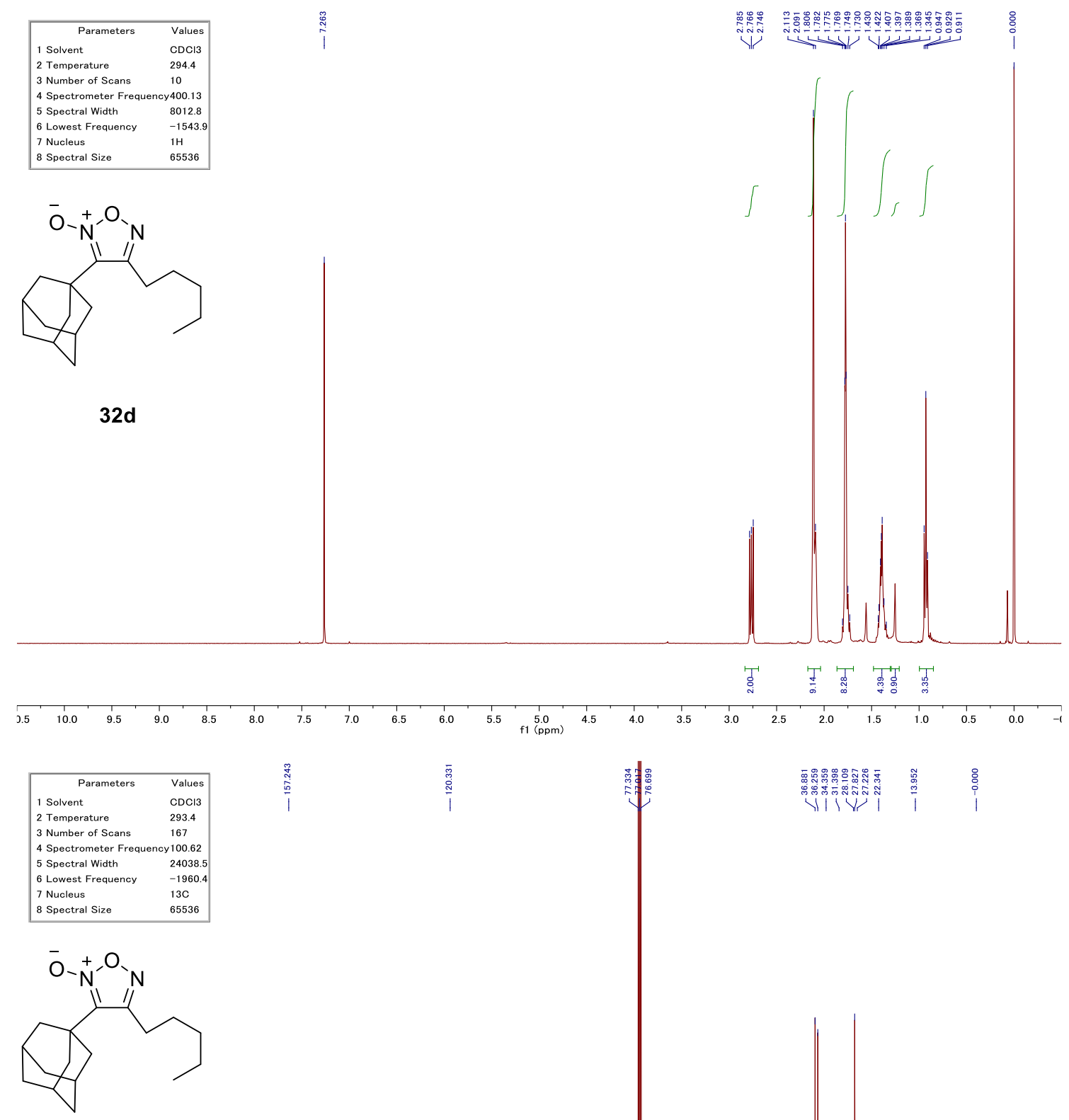

32d 